\title{
Republic of Armenia: First Review Under the Stand-By Arrangement, Request for Augmentation, Rephasing of Purchases, Waiver of the Nonobservance of Performance Criteria, and Modification of Performance Criteria—Staff Report; Staff Supplement; Press Release on the Executive Board Discussion; and Statement by the Executive Director for the Republic of Armenia.
}

In the context of the first review under the Stand-By Arrangement, request for augmentation, rephasing of purchases, waiver of the nonobservance of performance criteria, and modification of performance criteria for the Republic of Armenia the following documents have been released and are included in this package:

- $\quad$ The staff report for the First Review Under the Stand-By Arrangement, Rephasing of Purchases, Waiver of the Nonobservance of Performance Criteria, and Modification of Performance Criteria, prepared by a staff team of the IMF, following discussions that ended on May 20, 2009, with the officials of the Republic of Armenia on economic developments and policies. Based on information available at the time of these discussions, the staff report was completed on June 12, 2009. The views expressed in the staff report are those of the staff team and do not necessarily reflect the views of the Executive Board of the IMF.

- A staff supplement of June 17, 2009, on the assessment of the risks to the Fund and the Fund's liquidity position.

- $\quad$ A Press Release summarizing the views of the Executive Board as expressed during its June 22, 2009, discussion of the staff report.

- $\quad$ A statement by the Executive Director for the Republic of Armenia.

The documents listed below have been or will be separately released.

Letter of Intent sent to the IMF by the authorities of the Republic of Armenia*

Memorandum of Economic and Financial Policies by the authorities of the Republic of Armenia* Technical Memorandum of Understanding*

Statistical Annex/Appendix

*Also included in Staff Report

The policy of publication of staff reports and other documents allows for the deletion of market-sensitive information.

Copies of this report are available to the public from

International Monetary Fund • Publication Services

$70019^{\text {th }}$ Street, N.W. • Washington, D.C. 20431

Telephone: (202) 623-7430 • Telefax: (202) 623-7201

E-mail: publications@imf.org Internet: http://www.imf.org

\section{International Monetary Fund \\ Washington, D.C.}





\title{
INTERNATIONAL MONETARY FUND
}

\author{
REPUBLIC OF ARMENIA
}

\section{First Review Under the Stand-By Arrangement, Request for Augmentation, Rephasing of Purchases, Waiver of the Nonobservance of Performance Criteria, and Modification of Performance Criteria}

\author{
Prepared by the Middle East and Central Asia Department \\ (In consultation with other departments) \\ Approved by Ratna Sahay (MCD) and Ranil Salgado (SPR)
}

June 12, 2009

- $\quad$ Background and program performance: Since the approval of the stand-by arrangement (SBA) on March 6, 2009 (Country Report No. 09/140), Armenia's economic outlook has worsened substantially compared to the SBA projections, and external financing needs in 2009-10 have widened considerably. Program performance has been broadly on track.

- $\quad$ Request for augmentation of access: In light of the large increase in Armenia's financing needs, the authorities have requested an augmentation of Fund resources in the amount of 180 percent of quota (SDR 165.6 million or about $\$ 258$ million), which would bring total access under the SBA to 580 percent of quota. The revised program maintains the original objectives of responding to the external outlook, strengthening confidence in the domestic currency and the banking sector, and protecting the poor, but would also allow an easing of monetary and fiscal policies to mitigate the severity of the crisis.

- $\quad$ Recent political developments: President Sargsyan's Republican party won the Yerevan municipal elections on May 31, but the conduct of the elections raised concerns about irregularities. Recent prospects of a reopening of the TurkishArmenian border have stalled as Turkey closely links this with the issue of the Armenian-occupied territories in Azerbaijan.

- $\quad$ Mission: A staff team visited Yerevan on May 7-20. The team comprised Mr. Bonato, Ms. Hijazi, Mr. Sdralevich, and Mr. Lewis (head) (all MCD), Mr. Darius (SPR), Ms. ElGanainy (FAD), and Ms. Saeju (MCM). Ms. Oomes, resident representative, assisted the mission. Ms. Sahay (MCD) joined some of the policy discussions. The mission met with the Prime Minister, the ministers of finance and economy, the Governor of the Central Bank of Armenia (CBA), and representatives of donor countries, the banking sector, and the business community. 


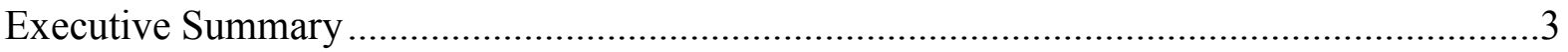

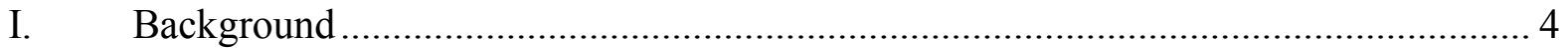

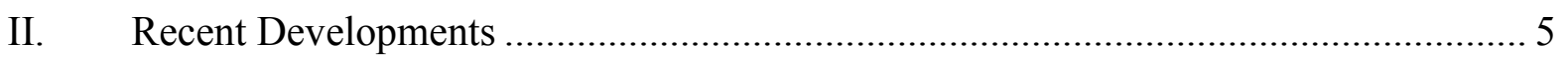

III. The Authorities' Program for 2009 and 2010 ......................................................... 7

A. Monetary and Exchange Rate Policies.......................................................... 9

B. Financial Sector Policies ...........................................................................

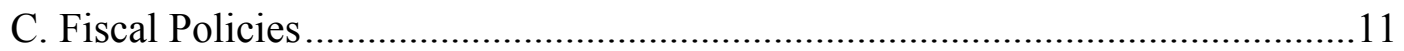

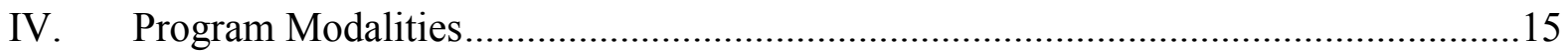

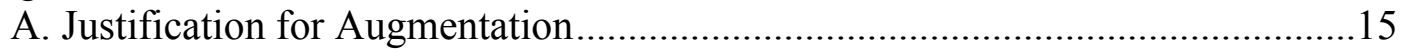

B. Augmentation Modality and Program Monitoring................................................17

C. Capacity to Repay the Fund ……………………..........................................17

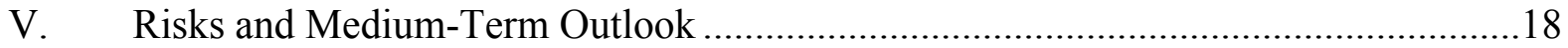

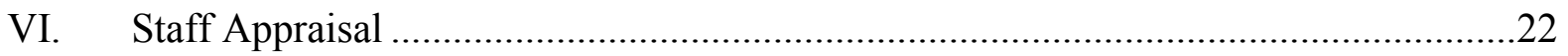

Boxes

1. Performance Under the SBA-Supported Program................................................... 4

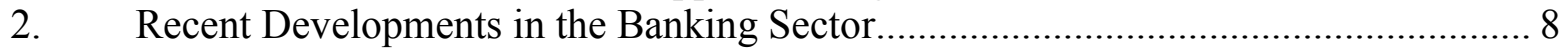

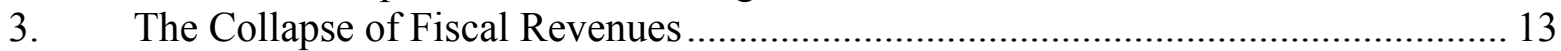

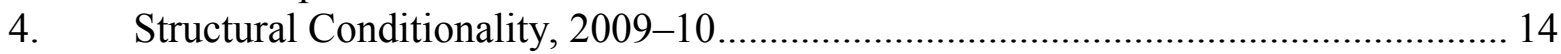

Figures

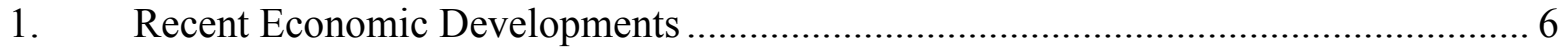

2. Public Debt Sustainability: Bound Tests ........................................................... 20

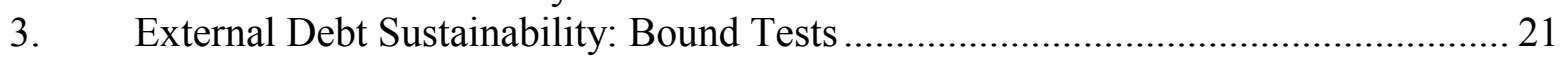

Tables

1. Selected Economic and Financial Indicators, 2006-10 ………............................2 25

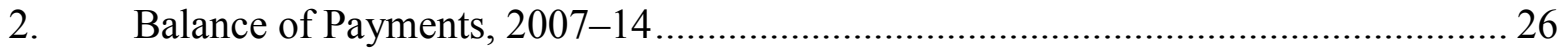

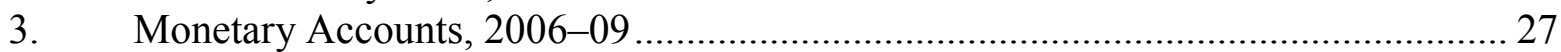

4. Financial Soundness Indicators for the Banking Sector, 2004-09 …...................... 38

5. Central Government Operations, 2007-10 …………………………………..... 29

6. Public Sector Debt Sustainability Framework, 2004-14 ……………………........ 31

7. External Debt Sustainability Framework, 2004-14 ............................................... 32

8. Medium-Term Macroeconomic Framework, 2007-14............................................ 33

9. Fund Disbursements and Timing of Reviews Under the Twenty-Eight

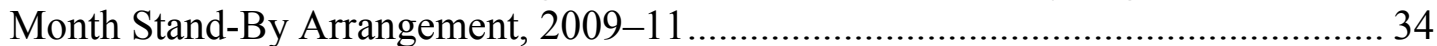

10. Indicators of Capacity to Repay the Fund, 2008-15 .................................................. 35

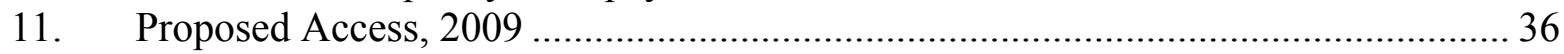

Attachments

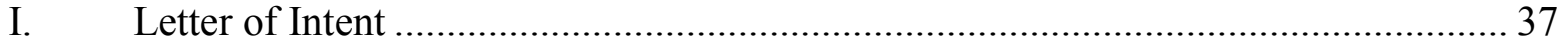

II. Memorandum of Economic and Financial Policies ................................................ 39

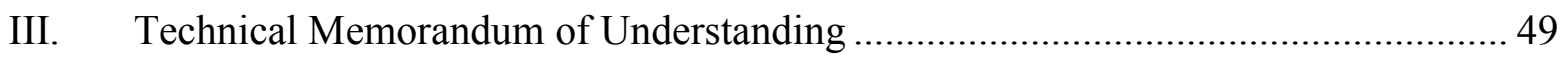




\section{EXECUTIVE SUMMARY}

Since the approval of the stand-by arrangement (SBA) on March 6, 2009, the economic outlook has deteriorated significantly. Staff now projects real GDP to contract by close to 10 percent in 2009 , compared to a fall of 1.5 percent projected at the time of the SBA request. External and fiscal financing needs have increased dramatically.

Despite the deteriorating fiscal situation, the program is broadly on track. While endMarch fiscal performance criteria were missed due to the large unexpected shortfall in tax revenues caused by the economic collapse, the monetary performance criteria were observed. In addition, the return to a floating exchange rate on March 3-a key element of the SBA program - has been successful. The 22 percent depreciation removed a significant exchange rate overvaluation with limited negative effects on the financial system.

\section{In light of the large increase in Armenia's financing needs, the authorities have} requested an augmentation of Fund resources in the amount of 180 percent of quota (SDR 165.6 million or about $\$ 258$ million), which would bring total access under the SBA to 580 percent of quota (SDR 533.6 million or about $\$ 830$ million).

The authorities have revised their SBA-supported program. To maintain the original SBA objectives of responding to the external outlook, strengthening confidence in the domestic currency and the banking sector, and protecting the poor, the revised program adjusts policies by:

- $\quad$ Easing monetary policy — consistent with the inflation-targeting framework - and implementing measures to increase its effectiveness, which has been weakened by the increase in deposit dollarization in the run-up to the depreciation. The authorities are also taking steps to address the ongoing credit crunch.

- $\quad$ Easing the fiscal stance by ringfencing key social programs and supporting development projects through additional external financing; and

- $\quad$ Laying the ground for future fiscal consolidation primarily through measures to strengthen tax policy and administration.

Fund exposure to Armenia is projected to increase over the medium term and there are important risks to the program, but Armenia's capacity to repay the Fund remains broadly good. Relatively low initial debt levels enable Armenia to increase external borrowings to mitigate the effects of the global crisis without unduly threatening mediumterm debt sustainability. However, significant risks remain. In the short term, the program could be affected in particular by a possible further deterioration in global economic conditions. In the medium term, the public debt is expected to be sustainable, thanks in part to planned fiscal consolidation, but will be vulnerable to unexpected shocks, particularly significantly lower-than-projected growth. 


\section{BACKGROUND}

1. On March 6, 2009 the Fund's Executive Board approved a SBA with exceptional access of 400 percent of quota, equivalent to SDR 368 million or about \$544 million (Country Report No. 09/140). The main objectives of the SBA-supported program were to achieve the necessary external adjustment, restore confidence in the domestic currency and the banking sector, and protect the poor.

\section{Since the approval of the SBA, the economic outlook has deteriorated}

considerably. The return to a floating exchange rate on March 3-a centerpiece of the SBAsupported program - has

been successful.

However, Armenia has

been strongly affected by

the global downturn,

notably through its

impact on Russia. As a

result, while program

implementation has

generally been good (Box

1), external and fiscal

\begin{tabular}{|c|c|c|c|c|c|}
\hline \multicolumn{6}{|c|}{$\begin{array}{c}\text { Armenia: Shortfall in Key Foreign Exchange Inflows } \\
\qquad \text { (millions of US dollars) }\end{array}$} \\
\hline & \multirow{2}{*}{$\begin{array}{l}2008 \\
\text { Prel. }\end{array}$} & \multicolumn{2}{|l|}{2009} & \multicolumn{2}{|l|}{2010} \\
\hline & & $\begin{array}{l}\text { Country Report } \\
\text { No. } 09 / 140\end{array}$ & Proj. & $\begin{array}{l}\text { Country Report } \\
\text { No. 09/140 }\end{array}$ & Proj. \\
\hline Exports & 1,078 & 815 & 703 & 962 & 801 \\
\hline Private Transfers & 1,062 & 820 & 704 & 869 & 718 \\
\hline FDI & 936 & 488 & 440 & 670 & 490 \\
\hline \multicolumn{6}{|l|}{ Memorandum items } \\
\hline Current account balance & $-1,346$ & $-1,229$ & $-1,215$ & $-1,081$ & $-1,209$ \\
\hline Armenia real GDP (percent change) & 6.8 & -1.5 & -9.5 & 3.0 & 1.3 \\
\hline Russia real GDP (percent change) & 5.6 & -0.7 & -6.0 & 1.3 & 0.5 \\
\hline
\end{tabular}

financing gaps have emerged in 2009-10 compared to projections at the time of the SBA approval. In response, the authorities are easing fiscal and monetary policies, implementing measures to improve credit conditions, raise tax revenue, and protect the poor, and reaching out for additional bilateral and multilateral financing to help support these policies and strengthen the international reserves position.

\section{Box 1. Armenia: Performance Under the SBA-Supported Program}

Policy implementation under the program was strong, but worse-than-anticipated economic conditions negatively affected some end-March quantitative targets (MEFP Table 1):

- $\quad$ Despite the postponement of non-essential spending, the authorities could not meet the endMarch performance criteria for the program fiscal balance and banking system net credit to the government, for which the authorities intend to request waivers. The reduction in outstanding tax credits progressed in line with the indicative target.

- $\quad$ Thanks to stable financial conditions after the March 3 depreciation, both the CBA's net domestic assets and net international reserves performance criteria were easily met. However, the reserve money indicative target was not met as banks increased their dollar reserves at the central bank in anticipation of the return to the floating exchange rate.

- $\quad$ There was no accumulation of external arrears.

- No structural conditionality was envisaged for end March. 
3. In this context, the authorities have requested an augmentation of access amounting to 180 percent of quota, equivalent to SDR 165.6 million, or around \$258 million. The additional resources would help close the newly-emerged financing gaps, bolster reserves, and maintain confidence. Given the potential pressure that an easing of fiscal policy would place on the balance of payments, it is anticipated that the resources from the augmentation, together with the June 2009 purchase, would be transferred directly to the government in 2009-2010. With the augmentation, total access under the arrangement would increase to 580 percent of quota. Annual access during the first year of the program is greater than 200 percent of quota, and thus would still qualify as exceptional under the new limits.

\section{RECENT DEVELOPMENTS}

4. Economic conditions have worsened further since the SBA approval. The economy is suffering from the drop in remittances, in particular from Russia, which had financed the boom in construction in recent years, notably of residential investment. Construction activity, which accounts for almost 30 percent of GDP, has contracted by 42 percent in the first four months of 2009 relative to the same period of 2008 , dragging down overall economic activity, which has fallen by close to 10 percent over the same period. Other sectors, notably mining, are facing significant difficulties in the face of weak external demand and low international commodity prices. In the second half of the year, as the planned easing of policies takes hold, the contraction is expected to level off with the launch of some large infrastructure projects, the increase in competitiveness following the exchange rate depreciation, and the recovery of international copper prices. For 2009 as a whole, real GDP is projected to contract by $9 \frac{1}{2}$ percent, and poverty is expected to increase. As global conditions improve, real GDP is projected to rise in 2010 , but only by about 1 percent (Table 1 ).

\section{The sharp economic downturn has caused a substantial deterioration of the external and fiscal positions:}

- $\quad$ Large drops in export receipts, remittances, and FDI have widened the gap in the external balance of payments despite the decline in imports associated with weaker economic activity (Table 2).

- $\quad$ Tax revenues have fallen by 15 percent in the first four months of 2009, and, despite a postponement of expenditures, the authorities were not able to meet the program targets related to the fiscal performance. 
Figure 1. Armenia: Recent Economic Developments

Remittances, Real Estate Prices and Growth

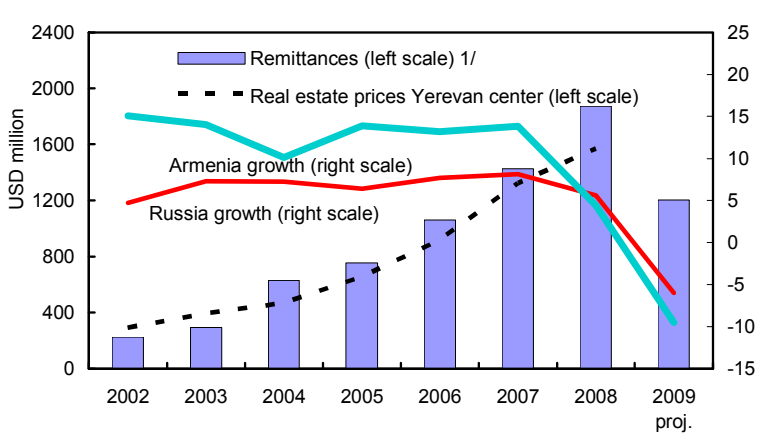

Current Account and Key Components (In percent of GDP)

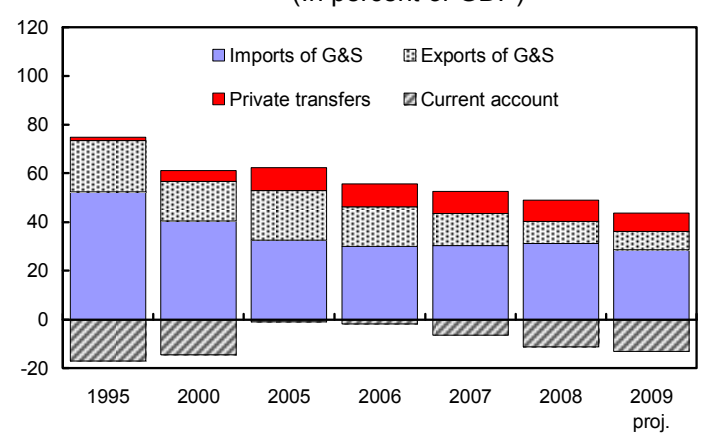

Real and Nominal Effective Exchange Rates (Index, 2000=100)

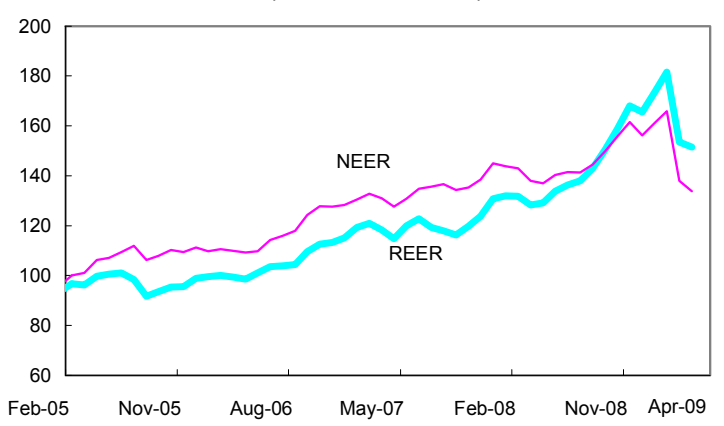

Remittance Inflows

(In percent of GDP)

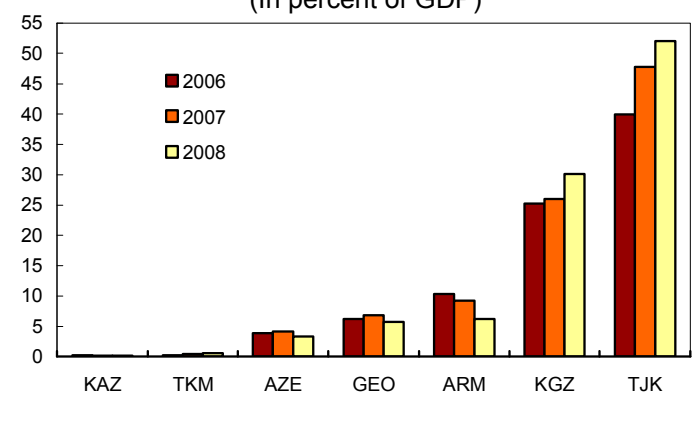

Capital and Financial Account and Key Components (In percent of GDP)

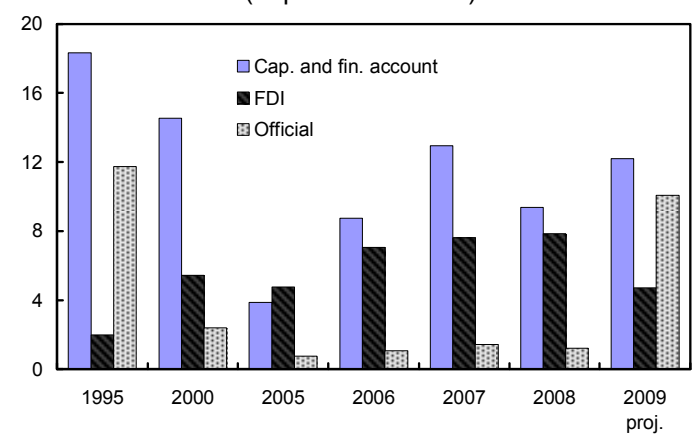

Fiscal Balance

(In percent of GDP)

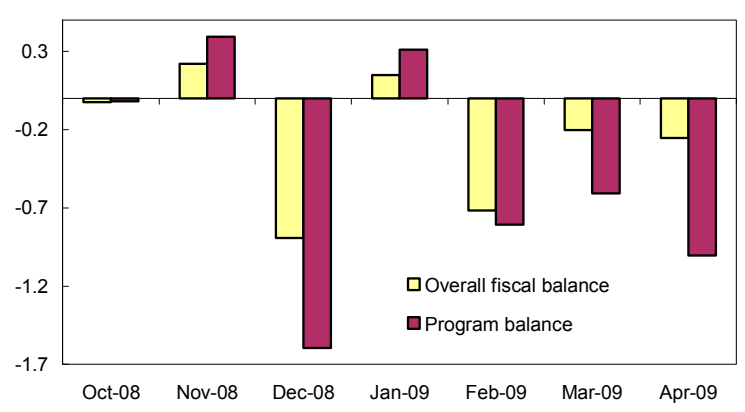

Sources: Armenian authorities; and Fund staff estimates.

$1 /$ Remittances are defined as the sum of compensation of employees, workers' remittances, and other nongovernment current transfers. 2008 figures are estimated. 
6. The 22 percent depreciation vis-à-vis the U.S. dollar on March 3 likely removed a significant exchange rate misalignment. ${ }^{1}$ Since the return to the floating exchange rate, transactions on the foreign

exchange market have declined considerably, and the CBA has intervened only on a few occasions - for a total net amount of 3.5 percent of gross international reserves - to smooth exchange rate volatility and facilitate the functioning of the market. Gross reserves were $\$ 1.2$ billion at end April. The financial sector remains solid, but credit conditions in the

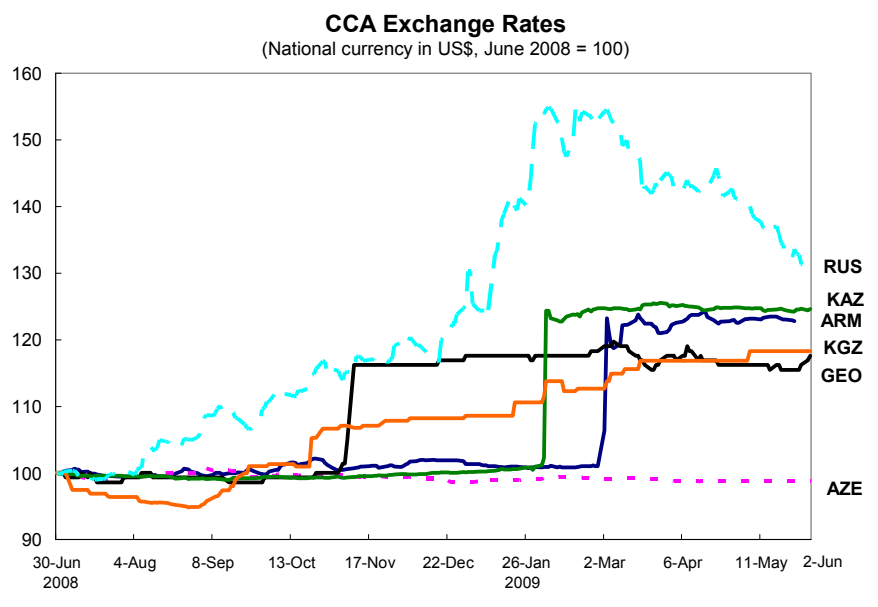
economy have tightened (Box 2).

7. Despite the depreciation, inflationary pressures remain muted. Annual CPI inflation rose to 3.4 percent in May from 1 percent in February and March, reflecting adjustments in utility tariffs and the pass-through from the March depreciation, which mostly affected imported food prices. Owing to ample spare capacity in the economy, inflation is expected to remain low throughout 2009.

\section{The AUthorities’’ Program fOR 2009 ANd 2010}

8. The authorities have revised their SBA-supported program in response to the worsening of the economic outlook for 2009-10. The revised program maintains the original objectives of the SBA-supported program, but adjusts policies to help offset the collapse in economic activity and lays the basis for the medium-term fiscal consolidation needed to address the build-up in public debt. Thus, the revised program focuses on:

- $\quad$ Easing monetary policy consistent with the inflation-targeting framework and taking measures to unblock bank lending;

- $\quad$ Easing the fiscal stance by ringfencing key social programs and supporting development projects through additional external financing;

\footnotetext{
${ }^{1}$ Staff estimates (available at $\mathrm{http}: / /$ www.imf.org/external/pubs/cat/longres.cfm?sk=22783.0) indicate that the exchange rate was overvalued by about 20-30 percent at the end of 2008, prior to the return to the floating exchange rate.
} 


\section{Box 2. Armenia: Recent Developments in the Banking Sector}

Following the return to the floating exchange rate, financial sector conditions have stabilized. Deposit dollarization has leveled off at around 67 percent after having rapidly increased from 35 percent in November 2008, as depositors converted their dram deposits into dollar deposits at a rapid pace in anticipation of the depreciation. Moreover, the depreciation did not trigger deposit outflows. With depositors converting part of their dollar receipts into drams, commercial banks were able to reduce their short foreign currency exposure.

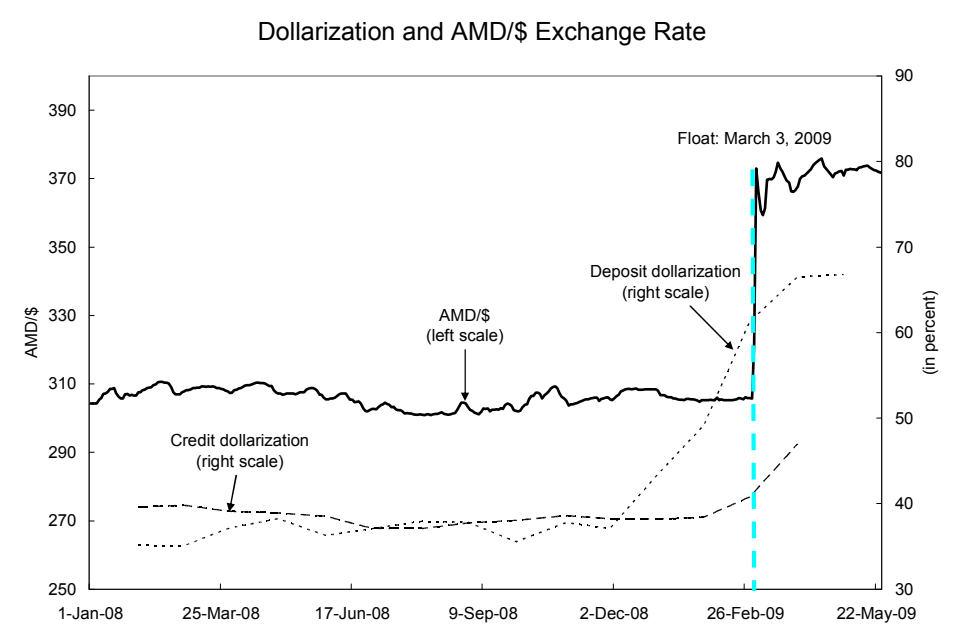

The banking system losses from the March 3 depreciation have been contained, also due to the small size of the banking sector relative to the economy. The first round valuation losses for those banks that had not managed to close their short foreign currency positions by March 3 are estimated at AMD 4.7 billion ( $\$ 12.6$ million) or around 15 percent of total 2008 profits and 0.2 percent of GDP.

The economic contraction is affecting the quality of loan portfolios. Nonperforming loans (NPLs) increased to 7.6 percent of gross loans at end March 2009 from 4.4 percent at end 2008. The increase was mainly due to a rise in loans in the "watch" category (up to 90 days past due)—which is likely to anticipate an increase in problem loans later in the year-as borrowers struggle to make payments on business and consumer loans (Table 4).

Despite these shocks, the banking system remains highly capitalized. The aggregate capital adequacy ratio (total regulatory capital to risk-weighted assets) declined from 27.5 percent at end 2008 to 26.1 percent at end March 2009, still more than twice the regulatory requirement of 12 percent.

However, new credit to the private sector has largely stopped. With high deposit dollarization and the resulting scarcity of long-term dram funding, banks are cutting back on their domestic currency assets, both by not rolling over dramdenominated loans and by restructuring them to dollar-denominated loans. At the same time, borrowers, whose income is mostly in domestic currency, are averse to borrowing in foreign currency and taking on the exchange rate risk. Consequently, adjusting for the valuation effect of the March 3 depreciation on the foreign currency loans (around half of the total), annual growth in credit to the private sector slowed down in the first quarter of 2009 to 25 percent compared to an average growth of 67 percent in 2008, and contracted by 5 percent from the last quarter

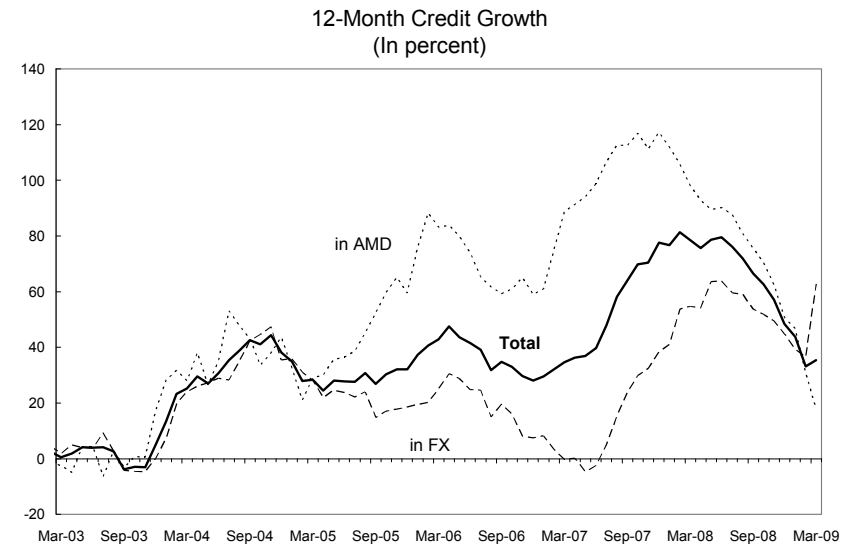
of 2008. Moreover, the number of new consumer loans in the first quarter has fallen by more than 40 percent year-on-year. 
- $\quad$ Laying the ground for future fiscal consolidation primarily through measures to strengthen the tax administration and address tax policy weaknesses.

\section{A. Monetary and Exchange Rate Policies}

\section{The authorities confirmed their commitment to the flexible exchange rate} regime. Since the March 3 depreciation, the CBA has intervened sparingly to smooth fluctuations in the foreign exchange market. The authorities remain committed to allowing the dram to move freely in line with the fundamentals. To this end they have agreed in the context of this review to adopt an exchange rate intervention strategy that will ensure consistency between monetary policy, liquidity

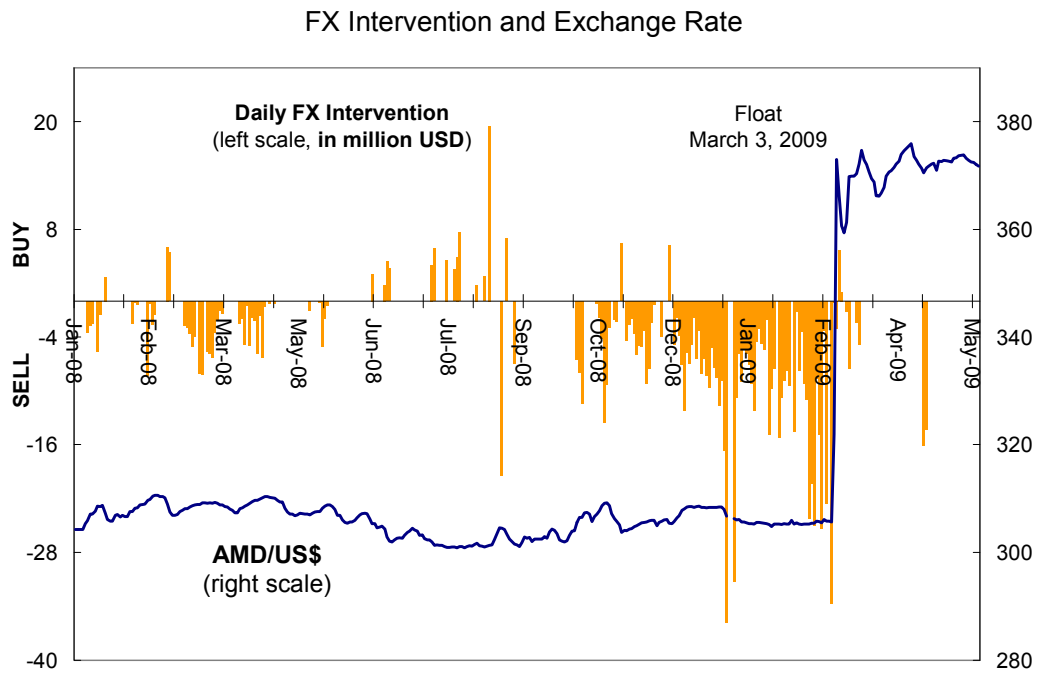
management and the flexible exchange rate regime (structural benchmark for end June 2009, MEFP, ๆ8).

10. The challenge for the $\mathrm{CBA}$ will be to address the uncertainty prevailing in the exchange rate market through its intervention and communication strategy. The CBA will need to find a balance between increasing the credibility of the float by allowing twosided movements of the exchange rate and providing some guidance to stabilize expectations in a thin market subject to large fluctuations. While market perceptions of further movements in the dram vary, recent agreements for large official financing, particularly the approval of the Fund SBA agreement in March and the signing of a \$500 million project loan from Russia in May, have alleviated concerns of a shortfall in foreign currency availability.

11. Monetary policy will again be guided by an inflation target. Reduced concerns about the exchange rate and financial stability have allowed the CBA to resume its inflation targeting policy (MEFP, 99). End-year inflation is on track to be at the lower end of the CBA's target range of $4 \pm 1.5$ percent.

12. Thus, the CBA intends to continue the recent easing of monetary policy. The CBA increased its policy rate by 100 basis points at the time of the return to the float to contain overshooting of the exchange rate and maintain confidence in the dram. However, since then, pressures on the exchange rate have abated, while concerns about economic activity have intensified. Therefore, since the beginning of April, the CBA has cut its policy 
rate in several steps by a cumulative 150 basis points, to 6.25 percent, and now expects to be able to cut rates further in the coming months, consistent with its inflation objective.

13. However, the normal channels of the transmission of monetary policy have considerably weakened. The steep increase in deposit dollarization in the run-up to the depreciation had already reduced the effectiveness of monetary policy, and the ongoing credit crunch poses new challenges. The authorities therefore see the need of taking steps to unblock credit to the private sector by strengthening monetary policy transmission and facilitate

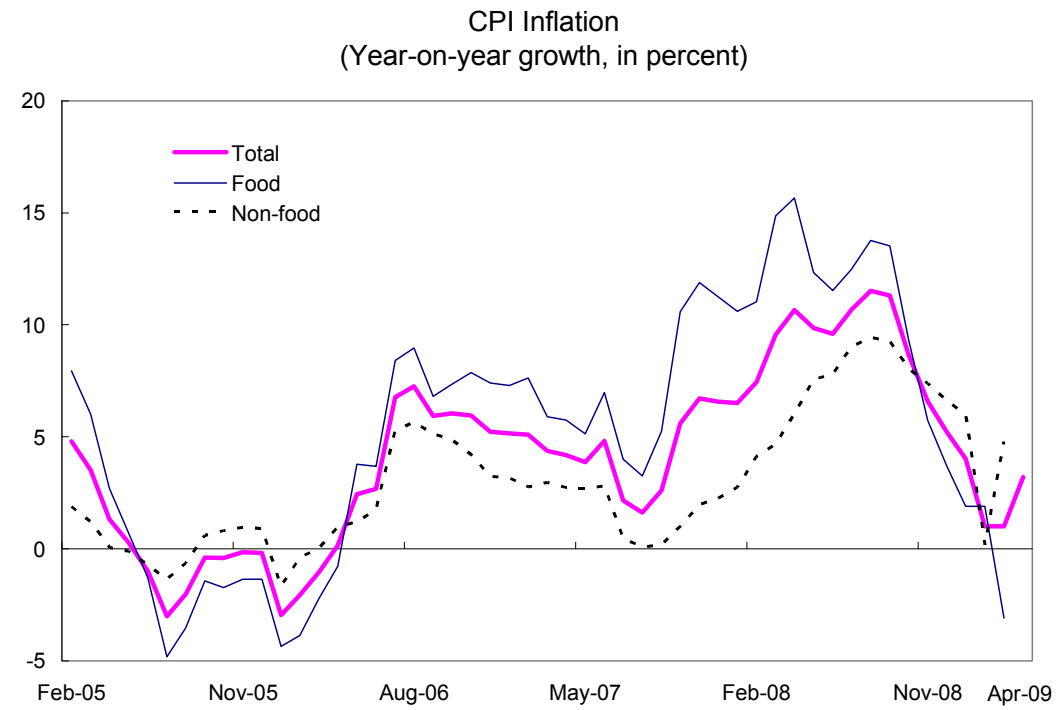
lending in domestic currency to the economy (MEFP, $\mid 11-13)$. In particular:

- $\quad$ The CBA is introducing additional repo instruments with longer maturities than the current 7-day and will purchase long-term government securities in the secondary market to provide longer-term dram liquidity to banks and facilitate lending in drams;

- Multilateral and bilateral donors (World Bank, the German development bank KfW, Russia) will provide financing to small and medium enterprises (SMEs) through the CBA and commercial banks;

- $\quad$ The CBA will implement exceptional credit easing operations, including by establishing a secondary mortgage operator with a mixed public-private capital envisaged at around AMD 25 billion (of which AMD 5 billion would be seed money from the CBA) that would provide long-term financing to commercial banks starting in 2010 .

\section{B. Financial Sector Policies}

\section{The authorities have considerably strengthened their crisis response capacity.}

The banking system has been growing in recent years but is small compared to the economy. At the same time, problems in the sector could have systemic implications, such as quasifiscal costs and shortages of credit. In anticipation of the return to the floating exchange rate in March, the CBA supplemented the wide array of tools at its disposal with instruments to inject emergency liquidity and facilitate mergers and acquisitions (MEFP, $\mid 15$ ). Moreover, the CBA intensified its monitoring of individual banks and has been conducting stress tests 
focusing on credit and market risk on a continuous basis (MEFP, $\uparrow 14$ ).

Although the feared loss of confidence in the banking system and related liquidity pressures did not materialize, the exercise sharpened the CBA's supervisory practice and crisis response capability.

\section{The CBA continues to} strengthen bank supervision, in

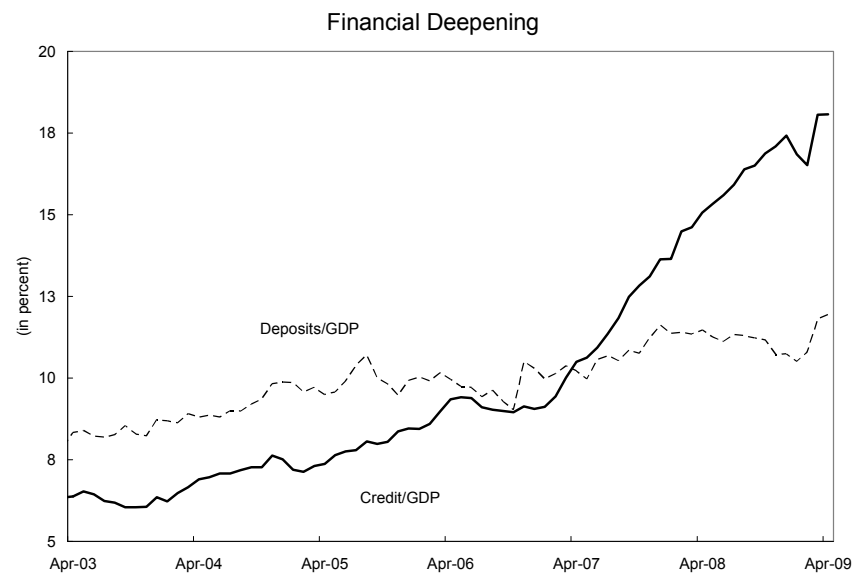
preparation for possible tail risks.

Aggregate bank capital is high but could be weakened by a rapid increase in NPLs and provisioning (made more likely by the currency mismatch between bank loans and borrowers' income) ${ }^{2}$ and by balance sheet losses caused by further exchange rate depreciation. Therefore, the CBA is continuing to closely monitor the banking system, and is considering tightening prudential regulations, including the reintroduction of a limit on open foreign exchange positions. In addition, the CBA intends to further bolster contingency planning, possibly with the help of Fund technical assistance. The authorities also intend to strengthen the bank resolution framework, and in this context are studying the adequacy of the Deposit Guarantee Fund (DGF) and are preparing options to increase its resources (structural benchmark for end June 2009, MEFP \16).

\section{Fiscal Policies}

16. To mitigate the impact of the crisis, the authorities intend to ease fiscal policy in

2009. This approach is guided by the expectation that the large shocks witnessed this year are mainly temporary and that the easing of the fiscal stance will help smooth the deep contraction. To this end, the program aims at largely compensating for the projected large drop in revenue through additional borrowing while preserving spending at the same level in nominal terms as in the original program. As a result, the fiscal deficit target for 2009 (including projects financed by the Russian loan) will be relaxed from 2.8 percent under the SBA to 6.5 percent of GDP.

17. Fiscal revenues are projected to fall by 1 percent of GDP (about 15 percent in nominal terms) relative to the projections at the time of the SBA (Box 3). Domestic borrowing options are scarce, as demand for government securities is limited and direct government borrowing from the central bank is forbidden by law. As a result, expenditure plans for the year have come under strong pressure. A consolidation of spending in line with

\footnotetext{
${ }^{2}$ According to CBA stress tests, a 25 percent write-off of NPLs would decrease the capital adequacy ratio of the system by 1.7 percent.
} 
the fall in revenues would add an additional contractionary impulse to the economy, further decreasing growth, and exacerbating the impact of the crisis on the poor.

\section{Expenditure plans focus on protecting social spending and shifting capital}

investment to foreign-financed projects. The program aims at preserving social spending at its budgeted levels, which would imply an increase of about 1.5 percent of GDP due to the lower nominal GDP. In anticipation of the increased demands on its social services brought about by the economic contraction, the authorities are also working closely with the World Bank to develop a strategy to further strengthen the targeting of social safety nets (structural benchmark for end December 2009, MEFP, $\mid 18)$. Capital spending, financed in part by the $\$ 500$ million project loan from Russia expected to be disbursed by end June, would be directed in particular to new housing projects in the earthquake zone (Gyumri, Vanadzor, and Spitak). The Russian loan would also fund SME lending via the banking system.

\section{In this context, financing needs would remain in 2009-10. Donor financing}

(including from Russia) and domestic financing, mainly from issuance of government securities, would leave fiscal financing needs of AMD 60 billion (1.8 percent of GDP) in 2009 and AMD 59 billion (1.7 percent of GDP) in 2010, notwithstanding the expected improvement of the fiscal deficit to 5.8 percent of GDP. The proposed augmentation of Fund resources, directed at mitigating the balance of payments pressures, would meet these needs.

\section{To strengthen the medium-term fiscal outlook, the authorities are committed to} an ambitious tax administration and policy reform agenda and to limiting the growth of spending. With the aim of improving the fiscal position starting in 2010 and opening up fiscal space to better serve the poor, the government has given priority to advancing its reform of tax administration, including by introducing best practices in VAT refund processing (continuous structural benchmark and structural benchmark for end September 2009, MEFP, q21) and of tax policy, notably by abolishing the presumptive taxation regime for tobacco and fuel in 2011 through legislation to be submitted to parliament by end 2009 (structural benchmark for end December 2009, MEFP, ๆ23). Over the medium term, the authorities aim to pare back nonpriority current spending, helping Armenia to adjust to a more subdued outlook and maintain debt sustainability while enhancing efforts to reduce poverty. 


\section{Box 3. Armenia: The Collapse of Fiscal Revenues}

Armenia is experiencing a large fall in fiscal revenues. The first-quarter 17 percent fall in tax revenues is largely due to the sharp decline in the two biggest tax categories in Armenia, i.e., VAT and profit tax, which fell by 20 percent and 21 percent respectively. The fall in VAT revenues reflects in large part a decline in imports, which account for more than twothirds of VAT collection.

Additional factors underlying the decline in revenues are:

- Increase in VAT noncompliance: Domestic VAT collection has fallen by 32 percent, while private

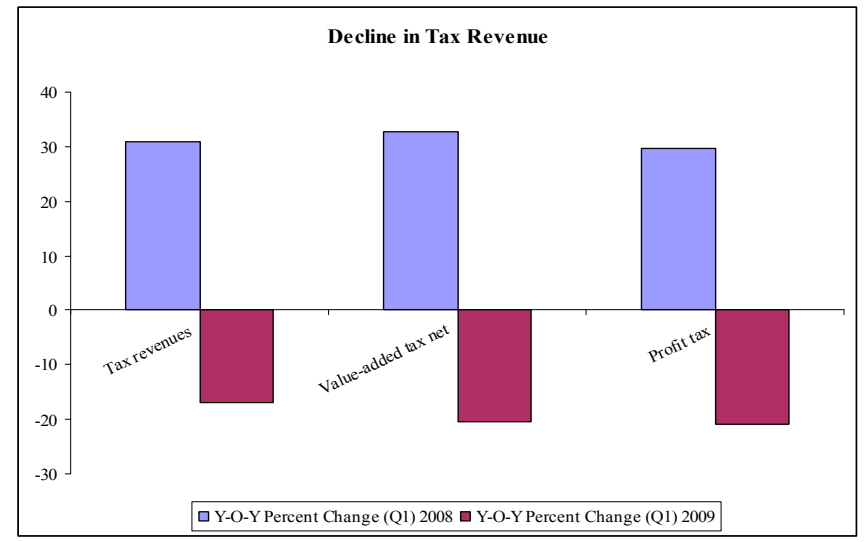
consumption growth is estimated to have been close to zero, suggesting a rise in non-compliance. The C-ratio (the ratio of net collected VAT to the maximum VAT recoverable on private consumption), a measure of the overall VAT collection efficiency, is estimated to have declined from around 66 percent at end 2007 to around 50 percent in the first quarter of 2009.

\section{- Impact of the crisis on large} tax payers: VAT and profit

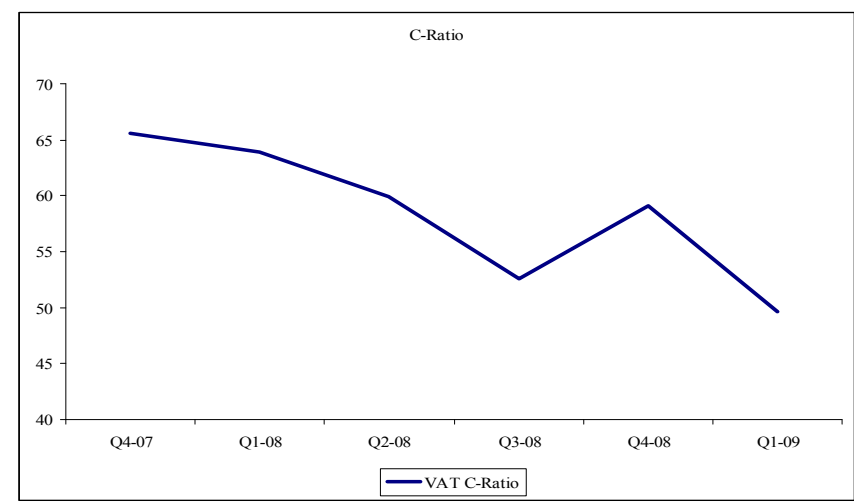
taxes from the 325 largest taxpayers (including the mining companies), which account for about 30 percent of total tax revenues, have fallen in the first quarter by 33 percent, equivalent to 73 percent of the total decline in tax revenues.

- Changes in tax policy: The threshold below which enterprises need not register for the VAT was set at AMD 58.3 million effective January 2009, leading to estimated revenue losses of up to AMD 6 billion ( 0.2 percent of GDP) by end 2009. However, the introduction of the threshold should lead to medium-term efficiency gains by reducing the cost of covering a large number of small businesses which do not yield significant revenue. 


\begin{tabular}{|c|c|}
\hline \multicolumn{2}{|c|}{ Box 4. Armenia: Structural Conditionality, 2009-10 } \\
\hline Structural Measure & Rationale for Macro-Criticality \\
\hline \multicolumn{2}{|l|}{ Financial Sector } \\
\hline $\begin{array}{l}\text { 1. Approve a plan to increase the resources of the Deposit } \\
\text { Guarantee Fund (structural benchmark for end June 2009). }\end{array}$ & $\begin{array}{l}\text { Boost the credibility of the DGF. } \\
\text { In case of bank closures, the Deposit Guarantee Fund would likely need further } \\
\text { resources to meet existing commitments to depositors }\end{array}$ \\
\hline $\begin{array}{l}\text { 2. CBA to adopt a formal foreign exchange market intervention strategy } \\
\text { consistent with IMF technical assistance (structural benchmark for end June } \\
\text { 2009). }\end{array}$ & $\begin{array}{l}\text { Ensure a coherent intervention strategy to achieve consistent and transparent } \\
\text { outcomes in line with the objectives of smoothing excessive movements of the } \\
\text { exchange rate and accumulating international reserves. }\end{array}$ \\
\hline $\begin{array}{l}\text { 3. Start offering repo operations with a maturity of at least three months } \\
\text { (structural benchmark for end June 2009). }\end{array}$ & $\begin{array}{l}\text { Unlock the frozen credit market and strengthen the transmission of monetary } \\
\text { policy by providing longer-term dram liquidity to facilitate banks' liquidity } \\
\text { management and promote domestic currency lending. }\end{array}$ \\
\hline $\begin{array}{l}\text { 4. Submit to parliament an amendment to Article } 11 \text { of the "Law on the } \\
\text { Central Bank of the Republic of Armenia" to ensure a gradual } \\
\text { recapitalization of the CBA with marketable interest bearing } \\
\text { securities. (structural benchmark for end September 2009). }\end{array}$ & $\begin{array}{l}\text { Promote central bank financing modalities that increase transparency and } \\
\text { support monetary policy implementation. }\end{array}$ \\
\hline \multicolumn{2}{|l|}{$\underline{\text { Tax Administration }}$} \\
\hline $\begin{array}{l}\text { 5. Meet the statutory 90-day processing deadline for all VAT refund } \\
\text { claims filed in } \mathbf{2 0 0 9} \text { (structural benchmark, continuous in 2009). }\end{array}$ & $\begin{array}{l}\text { Boost the integrity of the tax system and enhance revenue performance by } \\
\text { increasing compliance and reducing incentives for tax evasion. }\end{array}$ \\
\hline $\begin{array}{l}\text { 6. Submit legislation to parliament to (a) strengthen penalties for overstated } \\
\text { VAT refund claims effective January } 2010 \text {, and (b) pay interest on all } \\
\text { legitimate VAT refund claims filed after December } 2009 \text { and not refunded } \\
\text { within } 90 \text { days, with interest to be paid out of VAT revenues (structural } \\
\text { benchmark for end September 2009). }\end{array}$ & $\begin{array}{l}\text { Enhance the efficiency of tax administration and improve revenue } \\
\text { performance while ensuring the integrity of the VAT system by reducing } \\
\text { incentives for fraud and corruption. }\end{array}$ \\
\hline \multicolumn{2}{|l|}{ Tax and Social Policy } \\
\hline $\begin{array}{l}\text { 7. Submit legislation to parliament to bring petroleum and tobacco } \\
\text { products within the regular tax regimes (excise tax, customs duties, profit } \\
\text { tax, and VAT), effective January } 2011 \text { (structural benchmark for end } \\
\text { December 2009), as part of the unified Tax Code. }\end{array}$ & $\begin{array}{l}\text { Establish a sustainable and effective revenue base by increasing revenue } \\
\text { collection from petroleum and tobacco products, while enhancing tax } \\
\text { efficiency and equity. }\end{array}$ \\
\hline $\begin{array}{l}\text { 8. Develop a strategy to further strengthen the targeting of social safety } \\
\text { nets (structural benchmark for end December 2009) in close collaboration with } \\
\text { the World Bank. }\end{array}$ & $\begin{array}{l}\text { Improve the targeting and efficiency of social safety nets so as to provide } \\
\text { additional assistance to the poor while minimizing costs. }\end{array}$ \\
\hline
\end{tabular}




\section{Program Modalities}

\section{A. Justification for Augmentation}

\section{Under the worsened economic outlook for 2009-10, Armenia's financing gaps}

have widened. The external financing gap for 2009-10 is projected at around $\$ 658$ million after accounting for identified financing of (i) the $\$ 500$ million project loan from Russia, (ii) World Bank financing of \$545 million in 2009-2012 (with high upfront IDA budget support disbursements of $\$ 60$ million in 2009), (iii) Asian Development Bank (ADB) financing of about $\$ 130$ million in 2009-10, from which at least $\$ 70$ million provided for direct budget support, and (iv) previouslyapproved, but not yet disbursed, IMF support. ${ }^{3}$ The gap $-\$ 356$ million more for 2009-10 compared to the original $\mathrm{SBA}^{4}$ — would be filled by additional IMF resources from the augmentation, with

Armenia: External Financing Requirements and Sources, 2008-2011 (In millions of U.S. dollars, unless otherwise indicated)

\begin{tabular}{|c|c|c|c|c|}
\hline & 2008 & 2009 & 2010 & 2011 \\
\hline & Prel. & \multicolumn{3}{|c|}{ Projections } \\
\hline Total requirements & 2270 & 2228 & 1969 & 1936 \\
\hline External current account deficit (exc. transfers) & 2483 & 1983 & 1982 & 1990 \\
\hline Debt amortization & 15 & 22 & 24 & 24 \\
\hline Gross international reserve accumulation & -254 & 200 & -58 & -100 \\
\hline IMF repurchases and repayments & 26 & 24 & 21 & 22 \\
\hline Available financing & 2270 & 2009 & 1529 & 1870 \\
\hline Capital account and current transfers & 2263 & 1771 & 1528 & 1868 \\
\hline Capital transfers (net) & 149 & 103 & 73 & 74 \\
\hline Foreign direct investment & 936 & 440 & 490 & 568 \\
\hline Public sector disbursements & 160 & 807 & 233 & 202 \\
\hline of which: World Bank & & 135 & 127 & 108 \\
\hline Russia & & 500 & $\ldots$ & $\ldots$ \\
\hline Asian Development Bank & & 98 & 41 & 10 \\
\hline Other capital $1 /$ & -119 & -347 & -41 & 79 \\
\hline Current transfers & 1138 & 767 & 774 & 944 \\
\hline Private & 1062 & 704 & 718 & 848 \\
\hline Official & 75 & 63 & 55 & 97 \\
\hline Exceptional financing & 7 & 239 & 2 & 2 \\
\hline IMF & 7 & 237 & 0 & 0 \\
\hline Change in arrears (+ increase) & 0 & 0 & 0 & 0 \\
\hline Debt relief & 0 & 2 & 2 & 2 \\
\hline Financing gap & 0 & 218 & 440 & 66 \\
\hline SBA already aprroved but not disbursed & & 112 & 139 & 56 \\
\hline Proposed augmentation & & 106 & 152 & 0 \\
\hline Unidentified financing & & 0 & 149 & 10 \\
\hline \multicolumn{5}{|l|}{ Memorandum item: } \\
\hline \multicolumn{5}{|l|}{ Additional financing gap compared } \\
\hline to Country Report No. 09/140 & & 106 & 250 & 0 \\
\hline
\end{tabular}

residual financing needs in 2010 to be covered by donors, possibly including the European Union.

22. In filling part of the new financing gaps, the requested augmentation would help Armenia preserve the original objectives of the program. Otherwise, Armenia would be forced into a severe external and domestic adjustment that would further worsen growth, threaten the implementation of the SBA-supported program, and impose sizeable cuts in

3 The Russian loan is expected to have a maturity of 15 years with a grace period of 5 years and interest rate of LIBOR plus 3 percent. The resources provided by the World Bank and ADB will be on highly concessional terms in 2009, and will include a blend of concessional and non-concessional financing in the remaining years.

${ }^{4}$ To compare the respective gaps, it is worth noting that the March 2009 purchase from the Fund of \$237 million was included in the financing gap under the original SBA, but is now included in financing. 
social spending, with a negative impact on the poor. The augmentation, together with the sizeable additional foreign assistance from donors, would also allow the CBA to build up an appropriate reserve buffer in the context of a highly uncertain outlook.

\section{Staff's assessment is that Armenia continues to meet the four criteria for} exceptional access:

- $\quad$ Criterion 1-The member is experiencing or has the potential to experience exceptional balance of payments pressure on the current account or the capital account, resulting in a need for Fund financing that cannot be met within the normal limits. Armenia is facing balance of payments pressures from the external current account, associated with a steep contraction in current and prospective export earnings and remittances, and the capital account, primarily from a sharp contraction in FDI.

- Criterion 2-A rigorous and systematic analysis indicates that there is a high probability that the member's public debt is sustainable in the medium term. Standard stress tests indicate that Armenia's public debt position would remain sustainable in the presence of a variety of shocks (Figure 2 and Table 6). Despite a moderate rise in recent years, the private external debt level, at about 5 percent of GDP, is limited, and possible contingent claims from private external debt remain low.

- Criterion 3-The member has prospects of gaining or regaining access to private capital markets within the timeframe when Fund resources are outstanding. While Armenia has received significant FDI, including in the banking sector, the government has not borrowed on international capital markets to date. Prior to the economic downturn, Armenia was well positioned to access international markets. Successful implementation of the Fund-supported program, along with the expected economic recovery, will provide the necessary conditions to restore prospects for accessing private capital markets within the next two-three years.

- $\quad$ Criterion 4-The policy program of the member provides a reasonably strong prospect of success, including not only the member's adjustment plans but also its institutional and political capacity to deliver that adjustment. Armenia has a long track record of sound macroeconomic management and successful implementation of Fund-supported programs, including in the context of the current SBA. The proposed policy package contained in the program, supplemented by reforms supported by the World Bank and other donors, will help address the current challenges. The program would place the economy on a sustainable recovery path and strengthen fiscal balances in the medium term through the deepening of reformsnotably in the tax administration area, the financial sector, and the private sector by improving the business climate. 


\section{B. Augmentation Modality and Program Monitoring}

24. The requested augmentation would increase Fund financing to $\mathbf{5 8 0}$ percent of quota (SDR 533.6 million, or about \$830 million) over the 28 -month SBA. The requested funding would cover the remaining external financing gap for 2009, and a large portion of the remaining gaps in 2010 and 2011.

25. Fund purchases would increase by SDR 65.87 million, or about $\$ 100$ million, in 2009 and by SDR 99.73 million, or about \$150 million, in 2010. The additional resources under the augmentation scheduled for 2009 would be disbursed in full, following Board completion of the first review of the SBA and the augmentation, together with the planned purchase under the original access amounting to SDR 36.80 million. The remaining balance of the augmentation would be purchased in 2010 in four equal purchases of SDR 24.93 million in parallel with the original schedule of four purchases of SDR 23.55 million.

26. The revised program entails additional conditionality. In line with the need to lay the ground for future fiscal consolidation, strengthen social spending, and improve the effectiveness of monetary policy, the authorities are committing to new structural benchmarks in the area of taxation, social policy, and monetary and exchange rate policy implementation (Box 4 and MEFP Table 2). Consistent with the recent changes to the Fund's conditionality framework, these measures would be in the form of structural benchmarks, while it is proposed that the existing structural performance criterion (plan to increase resources of the DGF) would be converted into a structural benchmark. It is also proposed to modify end-June, end-September, and end-December quantitative performance criteria in line with the revised macroeconomic outlook. The authorities and staff also agreed to eliminate the indicative target on reserve money in view of the instability of money demand and the reduced concerns regarding inflation.

27. The program will continue to be monitored via quarterly quantitative performance criteria, indicative targets, and structural benchmarks. The quantitative performance criteria and indicative targets and the structural benchmarks are listed in Tables 1 and 2 of the MEFP. The second review of the program is expected to be completed on or after August 15, 2009, the third review on or after November 15, 2009, and the fourth review on or after February 15, 2010.

\section{Capacity to Repay the Fund}

28. Overall Fund exposure to Armenia is projected to increase over the medium term. Fund debt service indicators peak in 2013, and decline thereafter (Table 10). Thus, total obligations to the Fund would increase from 2.3 percent of exports in 2009 to a peak of 14 percent of exports in 2013 (81 percent of total debt service) but fall sharply to 4.6 percent of exports shortly thereafter (in 2015). Outstanding credit to the Fund would reach a maximum of 9.3 percent of GDP in 2010 but decline to less than 1 percent of GDP by 2015 . 
29. Armenia's capacity to repay the Fund broadly remains good. Relatively low initial debt levels enable Armenia to increase external borrowings to mitigate the effects of the global crisis without unduly threatening medium-term debt sustainability. The rise in repurchase obligations to the Fund during the period 2012-2013 is significant but temporary, reflecting the relatively short maturity profile of Fund lending and the relatively large proportion of the outstanding obligations to the Fund relative to total obligations.

\section{Risks ANd Medium-Term OUTLOOK}

30. In the short term, there are significant risks to the program arising from a worsening of global conditions and domestic factors:

- $\quad$ Risks to economic growth projections in 2009-10 appear evenly balanced, but uncertainty is high. A strong policy response might alleviate the sharp economic downturn, but Armenia is very vulnerable to a further deterioration of global conditions, particularly in Russia.

- $\quad$ Tax revenues could fall more than expected, especially in case of deepening economic contraction, and could undermine the fiscal program.

- $\quad$ Balance of payment inflows could turn out to be lower than projected, and the current account could deteriorate further_ possibly from higher oil prices_implying increased risks to the international reserves target and forcing further adjustment in the exchange rate.

- Uncertainty about economic conditions, including expectations regarding the appropriate level of the exchange rate, could worsen the negative feedback between the credit crunch and growth.

31. In the medium term, the

macroeconomic situation is expected to improve, but public debt will increase. As global growth resumes, Armenia is expected to gradually move toward a sustainable growth rate of 4-5 percent (Table 8), lower than the growth rates-fueled by construction activity-seen in the recent past. Following the deterioration experienced in recent years, the current account deficit will narrow steadily and international reserves will settle at a comfortable level. With the need for a fiscal stimulus coming to an end, the authorities will be able to strengthen the 
fiscal position by slowing spending growth and raising tax revenues. But the rise in external borrowing in 2009-10, particularly from the Fund, the World Bank, and Russia, will cause public debt to rise rapidly from the current low levels and peak in 2011 at about 46 percent of GDP, before falling to under 40 percent in 2013.

32. The debt dynamics are sustainable but remain vulnerable to several shocks. The projected debt-to-GDP levels do not appear excessive, and standard stress tests show that Armenia's debt remains sustainable (Figures 2 and 3 and Tables 6 and 7). However, rollover risk is high, mainly due to the relatively short maturity of Fund financing. In addition, standard stress tests indicate that the projected increase in borrowing has increased Armenia's vulnerability to growth and depreciation shocks which, under a broad range of assumptions, could increase the debt-to-GDP ratio to above 50 percent in the coming years. Finally, the debt dynamics would be affected by the emergence of contingent liabilities, in particular related to the introduction of the funded pension pillar.

\section{Growth prospects need to be supported by a wide-ranging program of structural}

reform. In addition to the positive effects on poverty and social indicators, future growth is a key factor in ensuring favorable debt dynamics, pointing to the need for a continuation of the authorities' extensive program of structural reform. In particular, improvements to the business environment would have the biggest payoff by increasing Armenia's attractiveness to foreign and domestic investment and promoting more broad-based private sector growth. Efforts in this direction might be hampered by resistance to change by vested interests. Finally, Armenia's growth outlook could greatly benefit from the opening of the border with Turkey. 
Figure 2. Armenia: Public Debt Sustainability: Bound Tests 1/

(Public debt, in percent of GDP)
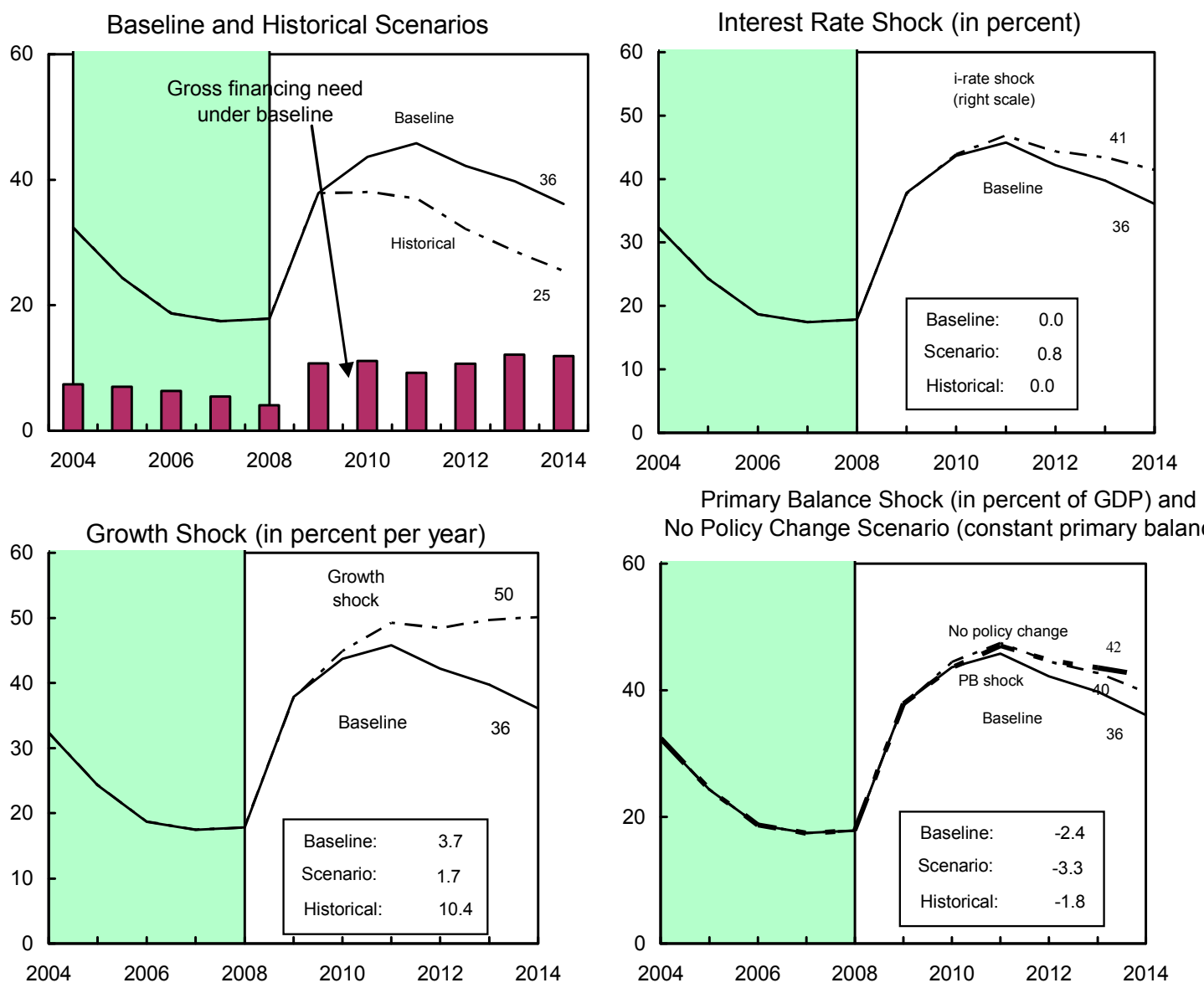

No Policy Change Scenario (constant primary balance)

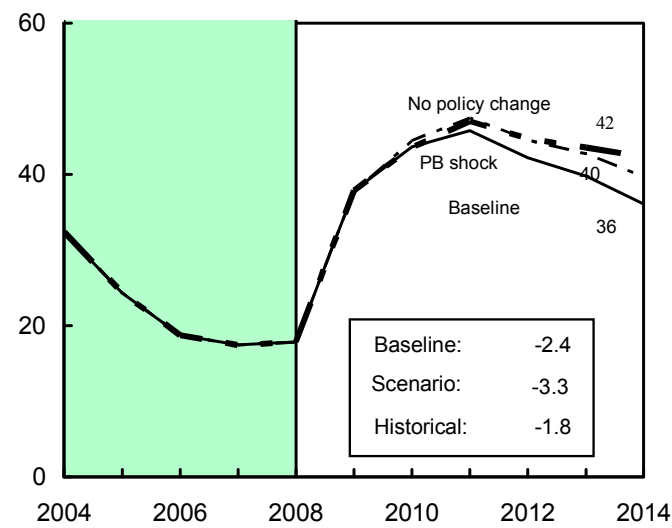

Combined Shock 2/

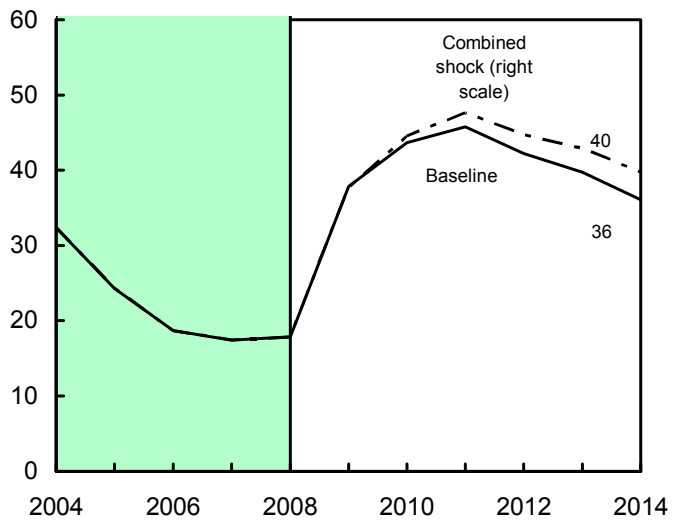

Real Depreciation and Contingent Liabilities Shocks 3/

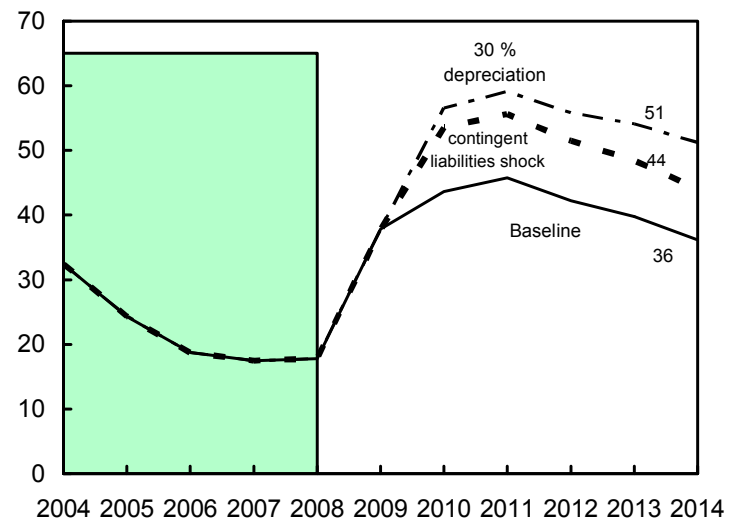

Sources: International Monetary Fund, country desk data, and staff estimates.

$1 /$ Shaded areas represent actual data. Individual shocks are permanent one-half standard deviation shocks. Figures in the boxes represent average projections for the respective variables in the baseline and scenario being presented. Ten-year historical average for the variable is also shown.

2/ Permanent $1 / 4$ standard deviation shocks applied to real interest rate, growth rate, and primary balance.

$3 /$ One-time real depreciation of 30 percent and 10 percent of GDP shock to contingent liabilities occur in 2009, with real depreciation defined as nominal depreciation (measured by percentage fall in dollar value of local currency) minus domestic inflation (based on GDP deflator). 
Figure 3. Armenia: External Debt Sustainability: Bound Tests 1/ (External debt, in percent of GDP)
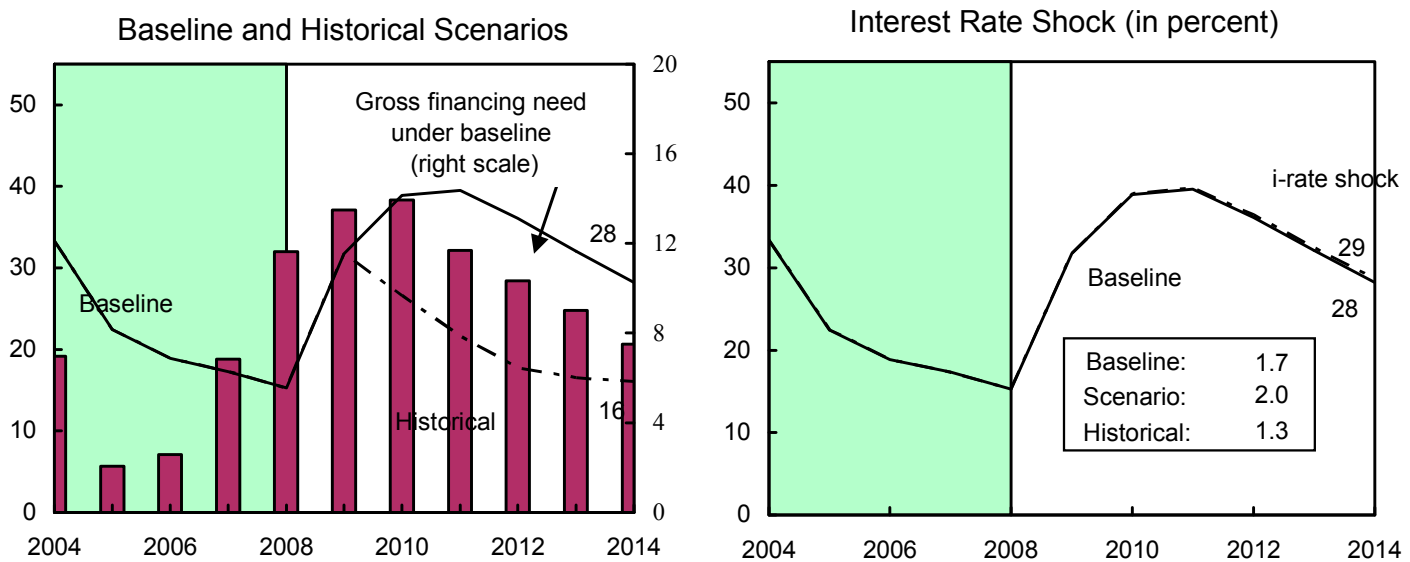

Growth Shock (in percent per year)

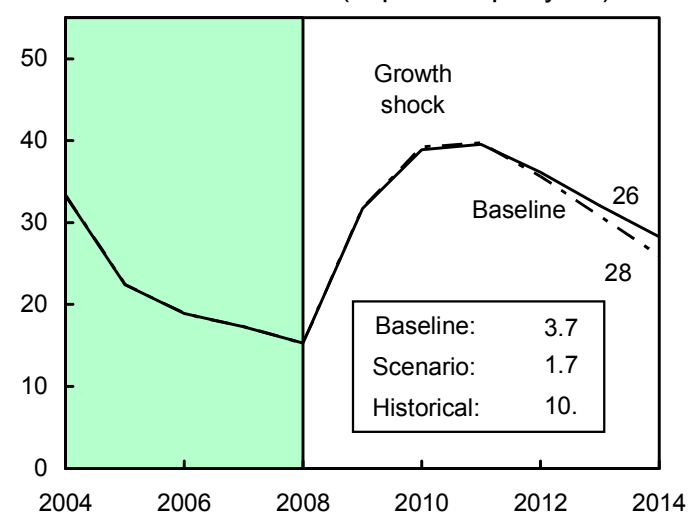

Non-Interest Current Account Shock (in percent of GDP)

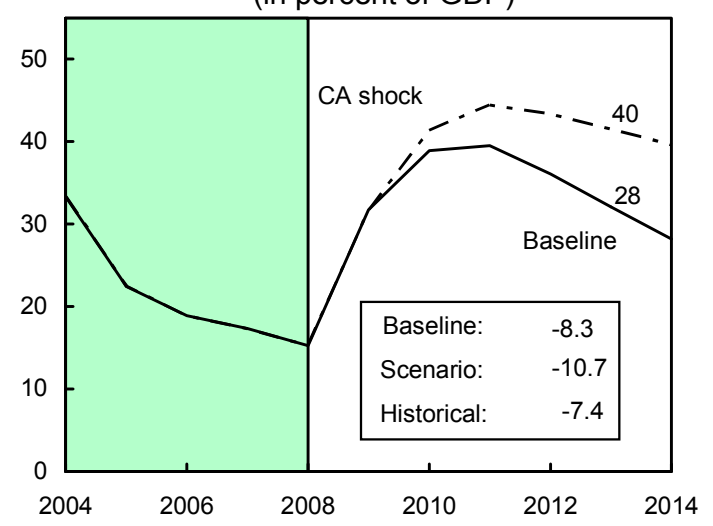

Combined Shock 2/

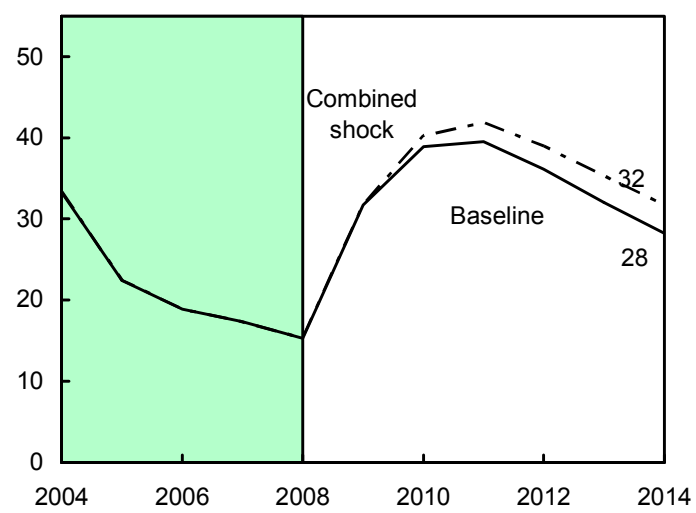

Real Depreciation Shock 3/

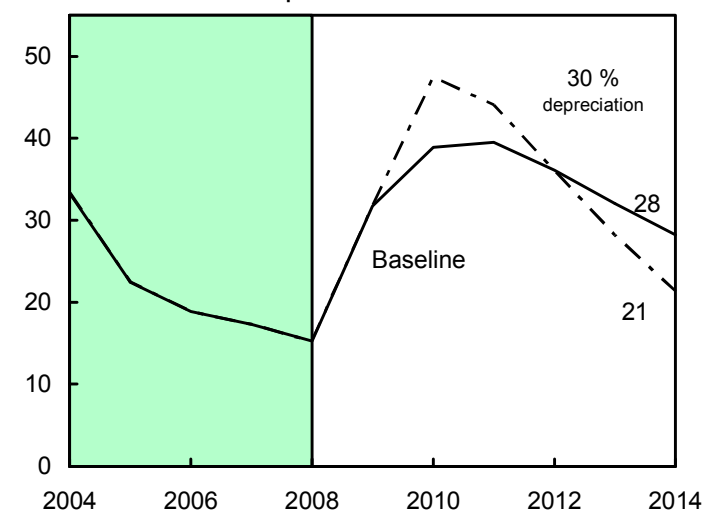

Sources: International Monetary Fund, Country desk data, and staff estimates.

1/ Shaded areas represent actual data. Individual shocks are permanent one-half standard deviation shocks. Figures in the boxes represent average projections for the respective variables in the baseline and scenario being presented. Ten-year historical average for the variable is also shown.

2/ Permanent $1 / 4$ standard deviation shocks applied to real interest rate, growth rate, and current account balance.

3/ One-time real depreciation of 30 percent occurs in 2009. 


\section{Staff Appraisal}

34. The economic outlook for Armenia has worsened considerably due to the global economic slowdown. Many of the downside risks identified at the time of the SBA request have materialized. In particular, the downturn in Russia is much deeper, and as a result foreign inflows have fallen dramatically. And with the deep economic contraction in Armenia, revenues have collapsed, opening up a sizable fiscal gap.

35. Despite these difficulties, policy implementation has generally been good. The return to the flexible exchange rate regime has thus far been a success, reversing what had become a significant overvaluation. Moreover, the authorities have so far successfully contained potentially destabilizing effects on the financial system. On public finances, faced with very serious revenue shortfalls, the authorities have thus far kept core government functions going but have compressed non-essential expenditures. Even so, key reforms continue to be implemented. To mitigate the sharp growth slowdown, the authorities are seeking additional financial support from international partners.

36. Looking ahead, the revisions to the authorities' program are appropriate in view of the very challenging situation. The original objectives of the program remain valid: help Armenia achieve the necessary external adjustment, maintain confidence in the currency and financial system, and protect the poor. Achieving these objectives, however, requires a reorientation of policies, notably an easing of monetary and fiscal policies, and targeted measures to ease credit conditions, raise revenues, facilitate private sector growth, and support social services.

\section{Continued exchange rate flexibility is a cornerstone of the authorities' policy} stance. Armenia is well-served by a flexible exchange rate regime. The return to a floating regime is welcome, and the authorities are encouraged to ensure that efforts to limit volatility are not interpreted by the market as a commitment to a particular level or range for the exchange rate. In this regard, careful, consistent, and frequent communication from the CBA is key.

38. With exchange rate and inflation pressures having receded, an easing of monetary policies is warranted. Consistent with the inflation targeting framework, a relaxation of monetary policy would help address the economic contraction - the authorities' steps in this direction thus far are both timely and welcome. In addition, more active measures to provide liquidity support to the financial system coupled with direct measures to unfreeze credit markets are needed to counteract what is an unfolding credit crunch that would otherwise threaten a deeper and more prolonged economic downturn.

39. Continued close supervision of the financial system is crucial. While the authorities' monitoring of the financial system is to be commended, the period ahead will be difficult - the banking sector remains highly capitalized, but nonperforming loans will likely continue rising in the coming months. The central bank is encouraged to continue 
strengthening its contingency planning and crisis preparedness, and, in this regard, the authorities' efforts to boost the Deposit Guarantee Fund resources are timely.

40. Some easing of fiscal policy is an appropriate response to mitigate the impact of the economic downturn. Compensating for the revenue drop through additional borrowing and maintaining spending in line with the original program will ensure that fiscal policy does not add to contractionary pressures. And increased spending on infrastructure and better targeted social spending will help boost growth and mitigate the impact of the crisis on the poor. At the same time, should the adverse global conditions turn out to be more permanent than temporary, fiscal policy would need to gradually adjust to a less robust economic outlook to prevent a further deterioration in the fiscal and debt dynamics.

\section{A strengthening of the tax administration reform program is central to the} authorities' response to the crisis and ensuring fiscal sustainability. To this end, introducing best practices in VAT administration, improved risk-based auditing, and other reforms should help lead to a sustained rise in revenue. Continued reductions in the stock of tax credits and overdue VAT refunds will be important to improving the integrity of the tax system and reducing the costs of the system to the private sector.

42. Likewise, continued structural reforms to boost the climate for private sector activity will be crucial for supporting growth, maintaining macroeconomic stability, and reducing poverty. Armenia's medium-term outlook is now more subdued, implying lower economic growth, less room for additional public borrowing, and slower poverty reduction. To this end, efforts to improve the business climate will be essential to boost domestic private and foreign direct investment, increase employment, and help spur a rapid and robust economic recovery.

43. Risks to the program have increased, even though the authorities are committed to strengthening and expanding the set of reform measures. The possibility of a significant worsening of economic and fiscal conditions cannot be ruled out. In addition, Armenia will have used up a large part of its borrowing space in a very short period of time. While debt stock indicators are expected to improve from 2011, debt sustainability could be cast into doubt if the fiscal position does not improve and/or growth does not rebound. Repayment risks to the Fund have likewise increased, although, with a still low debt service burden and a strong repayment record, Armenia is expected to meet its payments to the Fund in a timely manner. To address the increased risks, the authorities are committed to implementing additional new measures in monetary policy implementation and tax policy and administration reform.

44. Staff supports the authorities' request for augmentation and completion of the first review under the stand-by arrangement. In addition, staff supports the waivers of performance criteria on net banking system credit to the government and the program fiscal balance, the modification of end-June, end-September, and end-December quantitative 
performance criteria, and the conversion of the end-June structural performance criterion on the Deposit Guarantee Fund into and end-June structural benchmark requested by the authorities. Policy implementation under the program has been strong despite the worsened economic situation, and the authorities have implemented corrective actions and adjustments to the program as needed. The proposed policy package under the revised SBA is appropriate to the difficult economic circumstances, and the risks to the program are manageable. 
Table 1. Armenia: Selected Economic and Financial Indicators, 2006-10

\begin{tabular}{|c|c|c|c|c|c|c|}
\hline & \multirow[t]{2}{*}{2006} & \multirow[t]{2}{*}{2007} & 2008 & \multirow{2}{*}{$\begin{array}{c}\frac{2009}{\text { Country Report }} \\
\text { No. 09/140 }\end{array}$} & 2009 & 2010 \\
\hline & & & Prel. & & \multicolumn{2}{|c|}{ Proj. } \\
\hline \multicolumn{7}{|l|}{ National income and prices } \\
\hline Real GDP (percent change) & 13.2 & 13.7 & 6.8 & -1.5 & -9.5 & 1.3 \\
\hline Gross domestic product (in billions of drams) & 2,656 & 3,149 & 3,646 & 3,757 & 3,350 & 3,487 \\
\hline Gross domestic product (in millions of U.S. dollars) & 6,384 & 9,206 & 11,917 & 10,823 & 9,333 & 8,980 \\
\hline Gross domestic product per capita (in U.S. dollars) & 1,982 & 2,853 & 3,685 & 3,327 & 2,857 & 2,722 \\
\hline $\mathrm{CPI}$ (period average; percent change) & 2.9 & 4.4 & 9.0 & 4.5 & 3.0 & 2.7 \\
\hline $\mathrm{CPI}$ (end of period; percent change) & 5.2 & 6.6 & 5.2 & 8.0 & 3.5 & 2.9 \\
\hline GDP deflator (percent change) & 4.6 & 4.2 & 8.4 & 4.0 & 1.5 & 2.8 \\
\hline Unemployment rate (in percent) & 7.5 & 7.0 & 6.3 & $\ldots$ & $\ldots$ & $\ldots$ \\
\hline Poverty rate (in percent) & 26.5 & 25.0 & $\ldots$ & $\ldots$ & $\ldots$ & $\ldots$ \\
\hline \multicolumn{7}{|l|}{ Investment and saving (in percent of GDP) } \\
\hline Investment & 36.3 & 38.6 & 41.7 & $\ldots$ & 35.8 & 36.5 \\
\hline National savings & 34.4 & 32.2 & 30.4 & $\ldots$ & 22.7 & 23.0 \\
\hline \multicolumn{7}{|l|}{ Money and credit (end of period) } \\
\hline Reserve money (percent change) & 41.1 & 50.9 & 5.3 & 3.2 & 10.7 & 14.1 \\
\hline Broad money (percent change) & 32.9 & 42.3 & 2.4 & 2.9 & 10.4 & 14.1 \\
\hline Commercial banks' 3-month lending rate (in percent) & 17.1 & 18.6 & 17.9 & $\ldots$ & $\ldots$ & $\ldots$ \\
\hline \multicolumn{7}{|l|}{ Central government operations (in percent of GDP) } \\
\hline Revenue and grants & 18.0 & 20.1 & 20.0 & 21.1 & 20.4 & 20.4 \\
\hline Of which: tax revenue & 14.5 & 16.0 & 16.4 & 16.2 & 15.6 & 16.0 \\
\hline Expenditure 1/ & 20.0 & 22.4 & 21.8 & 23.9 & 26.9 & 26.3 \\
\hline Overall balance on a cash basis & -2.1 & -2.2 & -1.3 & -2.8 & -6.5 & -5.8 \\
\hline Overall balance (excl. projects related to the Russian loan) & $\ldots$ & $\ldots$ & $\ldots$ & $\ldots$ & -5.8 & -4.3 \\
\hline Government and government-guaranteed debt & 18.7 & 17.5 & 17.8 & 24.2 & 37.8 & 43.7 \\
\hline Share of foreign currency debt (in percent) & 88.2 & 88.1 & 85.8 & 90.8 & 90.0 & 89.8 \\
\hline \multicolumn{7}{|l|}{ External sector } \\
\hline Exports of goods and services (in millions of U.S. dollars) & 1,510 & 1,777 & 1,724 & 1,385 & 1,273 & 1,485 \\
\hline Imports of goods and services (in millions of U.S. dollars) & $-2,536$ & $-3,589$ & $-4,678$ & $-3,785$ & $-3,516$ & $-3,746$ \\
\hline Exports of goods and services (percent change) & 6.7 & 17.6 & -3.0 & -21.3 & -26.2 & 16.7 \\
\hline Imports of goods and services (percent change) & 19.4 & 41.5 & 30.3 & -19.9 & -24.8 & 6.5 \\
\hline Current account balance (in percent of GDP) & -1.8 & -6.4 & -11.3 & -11.4 & -13.0 & -13.5 \\
\hline FDI (net, in millions of U.S. dollars) & 450 & 701 & 936 & 488 & 440 & 490 \\
\hline External debt (in percent of GDP) /2 & 18.9 & 17.3 & 15.3 & 21.1 & 31.7 & 38.9 \\
\hline Debt service ratio (in percent of exports of goods and services) & 3.9 & 2.9 & 3.2 & 4.2 & 5.8 & 6.4 \\
\hline Gross international reserves (in millions of U.S. dollars) 3/ & 1,072 & 1,659 & 1,405 & 873 & 1,604 & 1,546 \\
\hline Import cover 4/ & 3.6 & 4.3 & 4.8 & 2.7 & 5.1 & 4.6 \\
\hline Nominal effective exchange rate (percent change) $5 /$ & 10.1 & 15.0 & 9.0 & $\ldots$ & $\ldots$ & $\ldots$ \\
\hline Real effective exchange rate (percent change) 5/ & 9.1 & 15.3 & 19.8 & $\cdots$ & $\cdots$ & $\ldots$ \\
\hline End-of-period exchange rate (dram per dollar) & 364 & 304 & 307 & $\ldots$ & $\ldots$ & $\ldots$ \\
\hline Average exchange rate (dram per dollar) & 416 & 342 & 306 & $\ldots$ & $\ldots$ & $\ldots$ \\
\hline \multicolumn{7}{|l|}{ Memorandum item: } \\
\hline Population (in millions) & 3.221 & 3.227 & 3.234 & $\ldots$ & $\ldots$ & $\ldots$ \\
\hline
\end{tabular}

Sources: Armenian authorities; and Fund staff estimates and projections.

$1 /$ Including the gas subsidy in 2006-2008.

2/ Based on government and government-guaranteed debt.

3/ Excluding the special privatization account (SPA), but including the Russian project loan.

4/ Gross international reserves in months of next year's imports of goods and services, including the use of Fund resources.

5/ A positive sign denotes appreciation. 
Table 2. Armenia: Balance of Payments, 2007-14

(in millions of U.S. dollars, unless otherwise indicated)

\begin{tabular}{|c|c|c|c|c|c|c|c|c|}
\hline \multirow[b]{3}{*}{ Current account } & \multirow{3}{*}{$\begin{array}{r}2007 \\
-589\end{array}$} & \multirow{3}{*}{$\begin{array}{l}\frac{2008}{\text { Prel. }} \\
-1,346\end{array}$} & 2009 & 2010 & 2011 & 2012 & 2013 & 2014 \\
\hline & & & \multicolumn{6}{|c|}{ Projections } \\
\hline & & & $-1,215$ & $-1,209$ & $-1,046$ & -854 & -660 & -606 \\
\hline Trade balance & $-1,600$ & $-2,630$ & $-1,966$ & $-2,052$ & $-2,137$ & $-2,211$ & $-2,277$ & $-2,327$ \\
\hline Exports, fob & 1,197 & 1,078 & 703 & 801 & 914 & 1,051 & 1,219 & 1,414 \\
\hline Imports, fob & $-2,797$ & $-3,708$ & $-2,669$ & $-2,854$ & $-3,051$ & $-3,261$ & $-3,496$ & $-3,741$ \\
\hline Services (net) & -213 & -325 & -277 & -209 & -182 & -160 & -176 & -181 \\
\hline Credits & 580 & 646 & 570 & 684 & 800 & 920 & 1,012 & 1,113 \\
\hline Debits & -793 & -971 & -847 & -893 & -982 & $-1,080$ & $-1,188$ & $-1,294$ \\
\hline Income (net) & 279 & 471 & 261 & 279 & 329 & 380 & 457 & 500 \\
\hline Transfers (net) & 945 & 1,138 & 767 & 774 & 944 & 1,137 & 1,337 & 1,402 \\
\hline Private & 850 & 1,062 & 704 & 718 & 848 & 1,059 & 1,293 & 1,396 \\
\hline Official & 94 & 75 & 63 & 55 & 97 & 77 & 44 & 6 \\
\hline Capital and financial account & 1,191 & 1,116 & 982 & 730 & 900 & 996 & 1,176 & 1,124 \\
\hline Capital transfers (net) & 143 & 149 & 103 & 73 & 74 & 72 & 62 & 62 \\
\hline Foreign direct investment (net) & 701 & 936 & 440 & 490 & 568 & 648 & 812 & 836 \\
\hline Portfolio investment (net) & -9 & 8 & 9 & 9 & 9 & 9 & 0 & 0 \\
\hline Public sector (net) & 133 & 145 & 785 & 208 & 178 & 167 & 177 & 156 \\
\hline Disbursements & 149 & 160 & 807 & 233 & 202 & 194 & 205 & 185 \\
\hline Amortization & -17 & -15 & -22 & -24 & -24 & -27 & -28 & -29 \\
\hline Other capital (net) & 224 & -121 & -356 & -50 & 70 & 100 & 125 & 70 \\
\hline Errors and omissions & -2 & -6 & 0 & 0 & 0 & 0 & 0 & 0 \\
\hline Overall balance & 600 & -235 & -234 & -479 & -147 & 142 & 516 & 519 \\
\hline Financing & -600 & 235 & 16 & 39 & 81 & -142 & -516 & -519 \\
\hline Gross international reserves (increase: -) & -587 & 254 & -200 & 58 & 100 & 11 & -220 & -252 \\
\hline Use of Fund credit, net & -14 & -19 & 214 & -21 & -22 & -155 & -298 & -269 \\
\hline Purchases/disbursements & 10 & 7 & 237 & $\ldots$ & $\ldots$ & $\ldots$ & $\ldots$ & $\ldots$ \\
\hline Repurchases/repayments & -24 & -26 & -24 & -21 & -22 & -155 & -298 & -269 \\
\hline Exceptional financing $1 /$ & 1 & 0 & 2 & 2 & 2 & 2 & 2 & 2 \\
\hline $\begin{array}{l}\text { Financing gap } \\
\text { Identified financing }\end{array}$ & 0 & 0 & 218 & 440 & 66 & 0 & 0 & 0 \\
\hline IMF :SBA approved & 0 & 0 & 112 & 139 & 56 & 0 & 0 & 0 \\
\hline IMF: proposed augmentation & $\ldots$ & $\ldots$ & 106 & 152 & $\ldots$ & $\ldots$ & $\ldots$ & ... \\
\hline Other & 0 & 0 & 0 & 149 & 10 & 0 & 0 & 0 \\
\hline \multicolumn{9}{|l|}{ Memorandum items: } \\
\hline Financing gap (Country Report No. 09/140) & 0 & 0 & 349 & 190 & 66 & 0 & 0 & 0 \\
\hline Current account (in percent of GDP) & -6.4 & -11.3 & -13.0 & -13.5 & -11.2 & -8.5 & -6.0 & -5.0 \\
\hline Trade balance (in percent of GDP) & -17.4 & -22.1 & -21.1 & -22.9 & -22.8 & -22.0 & -20.8 & -19.4 \\
\hline Gross international reserves (end of period) & 1,659 & 1,405 & 1,604 & 1,546 & 1,446 & 1,434 & 1,655 & 1,906 \\
\hline In months of next year's imports & 4.3 & 4.8 & 5.1 & 4.6 & 4.0 & 3.7 & 3.9 & 4.1 \\
\hline Gross international reserves excl. Russian project loan (end of period) & $\ldots$ & $\ldots$ & 1,179 & 1,246 & 1,196 & $\ldots$ & $\ldots$ & $\ldots$ \\
\hline In months of next year's imports & $\ldots$ & $\ldots$ & 3.8 & 3.7 & 3.3 & $\ldots$ & $\ldots$ & $\ldots$ \\
\hline Net international reserves (program definition) & 1,350 & 1,085 & 840 & 809 & 757 & 751 & 866 & 998 \\
\hline Merchandise export growth, percent change & 16.7 & -9.9 & -34.8 & 14.0 & 14.0 & 15.0 & 16.0 & 16.0 \\
\hline Merchandise import growth, percent change & 45.6 & 32.6 & -28.0 & 6.9 & 6.9 & 6.9 & 7.2 & 7.0 \\
\hline Nominal external debt $2 /$ & 1,591 & 1,818 & 2,962 & 3,493 & 3,697 & 3,628 & 3,508 & 3,394 \\
\hline Nominal external debt stock (in percent of GDP) 2/ & 17.3 & 15.3 & 31.7 & 38.9 & 39.5 & 36.1 & 32.1 & 28.2 \\
\hline External debt-to-exports ratio (in percent) $2 /$ & 89.6 & 105.5 & 232.7 & 235.2 & 215.7 & 184.1 & 157.2 & 134.3 \\
\hline External debt service in percent of exports $2 /$ & 2.9 & 3.2 & 5.8 & 6.4 & 6.2 & 12.4 & 17.3 & 14.0 \\
\hline
\end{tabular}

Sources: Armenian authorities; and Fund staff estimates and projections.

1/ Debt relief from the United Kingdom through 2015 (in respect of IDA credits).

2/ Based on government and government-guaranteed debt. 
Table 3. Armenia: Monetary Accounts, 2006-09

(In billions of drams, unless otherwise indicated)

\begin{tabular}{|c|c|c|c|c|c|c|c|c|c|c|c|c|c|}
\hline & $\frac{2006}{\text { Dec. }}$ & \multicolumn{4}{|c|}{2007} & \multicolumn{4}{|c|}{2008} & \multicolumn{4}{|c|}{2009} \\
\hline \multicolumn{14}{|l|}{ Central Bank of Armenia } \\
\hline Net foreign assets & 321.8 & 317.8 & 352.8 & 385.2 & 516.1 & 423.1 & 417.1 & 430.6 & 383.2 & 336.1 & 540.1 & 505.5 & 473.4 \\
\hline Net international reserves & 329.9 & 326.1 & 361.0 & 395.2 & 526.4 & 434.8 & 429.0 & 442.4 & 395.6 & 352.0 & 563.7 & 531.0 & 498.8 \\
\hline Medium and long-term & -8.0 & -8.3 & -8.2 & -10.0 & -10.3 & -11.6 & -12.0 & -11.9 & -12.4 & -15.9 & -23.6 & -25.5 & -25.5 \\
\hline Net domestic assets & -38.8 & -53.0 & -65.5 & -52.2 & -89.0 & -42.3 & -4.4 & 7.8 & 66.6 & 55.6 & -117.8 & -60.0 & 24.5 \\
\hline Claims on general government (net) & -57.7 & -66.6 & -93.8 & -87.9 & -89.8 & -124.7 & -121.6 & -108.7 & -96.5 & -60.3 & -280.6 & -243.9 & -209.5 \\
\hline Of which: central government (net) & -16.2 & -30.2 & -39.9 & -32.6 & -51.5 & -90.7 & -82.3 & -72.5 & -79.6 & -41.8 & -262.1 & -225.3 & -190.9 \\
\hline Claims on banks & 7.9 & 15.4 & 13.3 & 23.8 & 24.6 & 30.4 & 41.9 & 37.0 & 80.6 & 75.2 & 117.9 & 134.8 & 179.8 \\
\hline $\mathrm{KfW}$ & 11.9 & 12.0 & 12.6 & 13.2 & 14.4 & 16.0 & 17.3 & 18.1 & 19.9 & 20.1 & 27.8 & 29.7 & 29.7 \\
\hline Monetary instruments (net) excluding CBA bills & -4.0 & 3.4 & 0.7 & 10.5 & 10.2 & 14.4 & 24.7 & 19.0 & 60.7 & 55.1 & 90.1 & 105.1 & 150.1 \\
\hline CBA bills $2 /$ & -39.0 & -52.1 & -45.3 & -49.9 & -49.0 & -36.0 & -17.7 & -9.4 & -1.4 & 0.0 & 0.0 & 0.0 & 0.0 \\
\hline Other items (net) & 50.0 & 50.4 & 60.3 & 61.7 & 25.1 & 87.9 & 93.0 & 88.8 & 83.8 & 40.8 & 44.9 & 49.1 & 54.2 \\
\hline Reserve money & 283.0 & 264.8 & 287.3 & 333.0 & 427.1 & 380.8 & 412.7 & 438.3 & 449.7 & 391.7 & 422.3 & 445.5 & 497.9 \\
\hline Currency issue & 226.8 & 216.1 & 241.9 & 278.6 & 350.3 & 325.8 & 343.3 & 361.7 & 344.5 & 234.1 & 253.2 & 275.5 & 297.6 \\
\hline Deposits & 56.2 & 48.7 & 45.5 & 54.4 & 76.8 & 55.1 & 69.4 & 76.6 & 105.2 & 157.7 & 169.1 & 170.1 & 200.3 \\
\hline \multicolumn{14}{|l|}{ Banking system } \\
\hline Net foreign assets & 332.5 & 322.9 & 353.4 & 353.3 & 369.6 & 347.7 & 344.0 & 290.2 & 229.2 & 124.2 & 332.7 & 301.1 & 272.0 \\
\hline Net domestic assets & 153.2 & 160.0 & 169.7 & 228.6 & 321.8 & 331.0 & 368.1 & 463.0 & 479.0 & 517.2 & 343.0 & 428.4 & 509.7 \\
\hline Claims on government (net) & -31.8 & -42.3 & -70.1 & -64.0 & -54.0 & -80.4 & -78.2 & -62.0 & -37.3 & -10.6 & -217.6 & -163.7 & -118.3 \\
\hline Of which: claims on central government (net) & 9.7 & -5.8 & -16.3 & -8.8 & -15.7 & -46.4 & -38.9 & -25.8 & -20.5 & 7.9 & -199.1 & -145.2 & -99.7 \\
\hline Claims on rest of the economy & 241.5 & 271.6 & 306.3 & 371.2 & 429.8 & 485.7 & 550.6 & 618.5 & 638.6 & 659.5 & 657.0 & 683.9 & 706.4 \\
\hline Other items (net) & -56.4 & -69.3 & -66.5 & -78.6 & -54.0 & -74.3 & -104.3 & -93.6 & -122.3 & -131.7 & -96.4 & -91.7 & -78.4 \\
\hline Broad money & 485.7 & 482.9 & 523.0 & 581.9 & 691.3 & 678.7 & 712.1 & 753.2 & 708.2 & 641.4 & 675.7 & 729.5 & 781.6 \\
\hline Currency in circulation & 211.5 & 201.7 & 227.0 & 260.3 & 326.0 & 302.5 & 320.5 & 334.1 & 316.1 & 212.3 & 230.4 & 251.7 & 272.8 \\
\hline Deposits & 274.3 & 281.3 & 296.0 & 321.6 & 365.3 & 376.1 & 391.6 & 419.1 & 392.2 & 429.1 & 445.3 & 477.9 & 508.9 \\
\hline Domestic currency & 130.9 & 145.4 & 158.4 & 186.3 & 235.0 & 236.4 & 246.4 & 270.2 & 219.5 & 143.7 & 144.6 & 164.1 & 180.6 \\
\hline Foreign currency & 143.4 & 135.8 & 137.6 & 135.3 & 130.3 & 139.8 & 145.2 & 148.9 & 172.6 & 285.5 & 300.7 & 313.7 & 328.3 \\
\hline \multicolumn{14}{|l|}{ Memorandum items: } \\
\hline Exchange rate (in drams per U.S. dollar, end of period) & 363.5 & 362.1 & 341.0 & 335.8 & 304.2 & 307.3 & 302.7 & 302.1 & 306.7 & 367.8 & $\ldots$ & $\ldots$ & 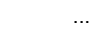 \\
\hline NIR, program definition, at program exchange rates (in millions of U.S. dollars) & 842.5 & 833.3 & 998.8 & $1,081.1$ & $1,350.1$ & $1,328.0$ & $1,279.6$ & $1,320.0$ & $1,084.7$ & 588.5 & $1,008.3$ & 923.3 & 839.8 \\
\hline NDA of the CBA (in billions of drams) & -38.8 & -53.0 & -65.5 & -52.2 & -89.0 & -42.3 & -4.4 & 7.8 & 66.6 & 55.6 & -117.8 & -60.0 & 24.5 \\
\hline 12-month change in reserve money (in percent) & 41.1 & 40.0 & 44.3 & 44.1 & 50.9 & 43.8 & 43.6 & 31.6 & 5.3 & 2.9 & 2.3 & 1.6 & 10.7 \\
\hline 12-month change in broad money (in percent) & 32.9 & 34.7 & 41.1 & 43.5 & 42.3 & 40.5 & 36.2 & 29.4 & 2.4 & -5.5 & -5.1 & -3.1 & 10.4 \\
\hline 12-month change in private sector credit (in percent) & 28.2 & 34.7 & 39.8 & 64.6 & 78.0 & 78.8 & 79.8 & 66.6 & 48.6 & 35.8 & 19.3 & 10.6 & 10.6 \\
\hline Velocity of broad money (end of period) & 5.5 & 5.6 & 5.4 & 5.1 & 4.6 & 4.7 & 4.7 & 4.8 & 5.1 & 5.6 & 5.3 & 4.7 & 4.3 \\
\hline Money multiplier & 1.7 & 1.8 & 1.8 & 1.7 & 1.6 & 1.8 & 1.7 & 1.7 & 1.6 & 1.6 & 1.6 & 1.6 & 1.6 \\
\hline Dollarization in bank deposits $3 /$ & 52.3 & 48.3 & 46.5 & 42.1 & 35.7 & 37.2 & 37.1 & 35.5 & 44.0 & 66.5 & 67.5 & 65.7 & 64.5 \\
\hline Dollarization in broad money $4 /$ & 29.5 & 28.1 & 26.3 & 23.3 & 18.9 & 20.6 & 20.4 & 19.8 & 24.4 & 44.5 & 44.5 & 43.0 & 42.0 \\
\hline Currency in circulation in percent of deposits & 77.1 & 71.7 & 76.7 & 80.9 & 89.2 & 80.4 & 81.8 & 79.7 & 80.6 & 49.5 & 51.7 & 52.7 & 53.6 \\
\hline Stock of foreign currency deposits (in millions of U.S. dollars) & 394.5 & 375.1 & 403.6 & 403.0 & 428.4 & 459.4 & 477.4 & 489.4 & 567.4 & 741.5 & 781.1 & 814.9 & 852.8 \\
\hline Banking system financing of the central government (cumulative) & -19.4 & -15.6 & -26.0 & -18.5 & -25.5 & -30.7 & -23.1 & -10.0 & -4.7 & 28.4 & -178.6 & -124.7 & -79.3 \\
\hline
\end{tabular}

Sources: Central Bank of Armenia; and Fund staff estimates and projections.

1/ At the program exchange rate.

2/ Following the agreement between the CBA and the Ministry of Finance, the issue of new CBA bills was terminated in 2008.

3/ Ratio of foreign currency deposits to total deposits (in percent).

4/ Ratio of foreign currency deposits to broad money (in percent). 
Table 4. Armenia: Financial Soundness Indicators for the Banking Sector, 2004-09

(In percent, unless otherwise indicated)

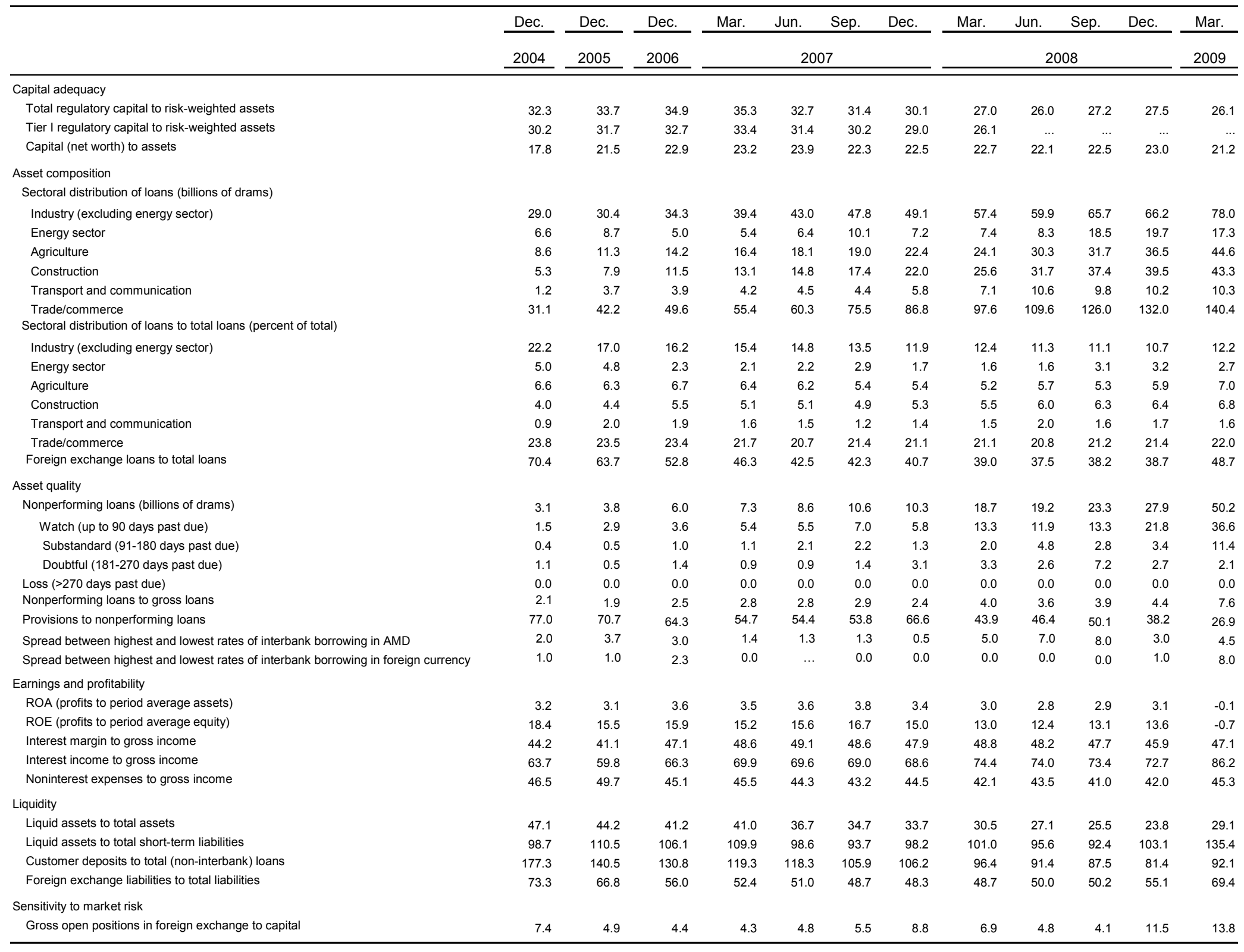

Source: Central Bank of Armenia. 
Table 5. Armenia: Central Government Operations, 2007-10

(In billions of drams)

\begin{tabular}{|c|c|c|c|c|c|c|c|c|c|}
\hline & \multirow{3}{*}{$\frac{2007}{\text { Jan.-Dec. }}$} & \multirow{3}{*}{$\begin{array}{c}2008 \\
\text { Jan.-Dec. } \\
\text { Prelim. }\end{array}$} & \multirow{2}{*}{\multicolumn{2}{|c|}{$\frac{2009}{\text { Jan.-Dec. }}$}} & \multicolumn{4}{|c|}{2009} & \multirow{3}{*}{$\begin{array}{c}\frac{2010}{\text { Jan.-Dec. }} \\
\text { Proj. }\end{array}$} \\
\hline & & & & & \multirow{2}{*}{$\begin{array}{c}\text { Q1 } \\
\text { Prel. }\end{array}$} & \multirow{2}{*}{$\begin{array}{c}\text { Q2 } \\
\text { Proj. }\end{array}$} & \multirow{2}{*}{$\begin{array}{c}\text { Q3 } \\
\text { Proj. }\end{array}$} & \multirow{2}{*}{$\frac{\text { Q4 }}{\text { Proj. }}$} & \\
\hline & & & IMF Proj. & Budget & & & & & \\
\hline Total revenue and grants & 632.5 & 730.8 & 682.4 & 879.4 & 134.9 & 173.7 & 182.8 & 191.0 & 713.0 \\
\hline Total revenue & 609.1 & 715.6 & 638.9 & 844.7 & 129.8 & 161.9 & 169.5 & 177.6 & 676.4 \\
\hline Tax revenues & 504.3 & 597.9 & 522.5 & 701.5 & 102.4 & 133.8 & 140.5 & 145.7 & 557.8 \\
\hline VAT & 248.0 & 296.3 & 251.7 & $\ldots$ & 47.1 & 62.4 & 68.3 & 73.9 & $\ldots$ \\
\hline Profits, simplified and presumptive & 103.4 & 113.3 & 95.9 & $\ldots$ & 21.5 & 27.6 & 22.9 & 24.0 & $\ldots$ \\
\hline Personal income tax & 46.8 & 53.7 & 51.0 & $\ldots$ & 12.0 & 11.8 & 13.4 & 13.8 & $\ldots$ \\
\hline Customs duties & 24.0 & 37.3 & 26.5 & $\ldots$ & 6.3 & 5.9 & 7.1 & 7.2 & $\ldots$ \\
\hline Other & 82.1 & 97.3 & 97.3 & $\ldots$ & 15.6 & 26.1 & 28.9 & 26.8 & $\ldots$ \\
\hline Social contributions & 85.1 & 104.1 & 101.6 & 128.4 & 21.5 & 24.5 & 25.1 & 30.5 & 107.0 \\
\hline Other revenue & 19.6 & 13.6 & 14.8 & 14.8 & 5.9 & 3.6 & 3.9 & 1.4 & 11.6 \\
\hline Grants & 23.5 & 15.1 & 43.5 & 34.7 & 5.0 & 11.8 & 13.3 & 13.4 & 36.6 \\
\hline Total expenditure & 706.1 & 793.4 & 899.5 & 919.4 & 158.1 & 247.5 & 245.1 & 248.8 & 916.7 \\
\hline Expense & 541.6 & 652.0 & 732.9 & 744.8 & 142.1 & 184.9 & 190.6 & 215.3 & 736.3 \\
\hline Wages $1 /$ & 61.3 & 73.0 & 88.9 & 61.6 & 14.9 & 24.5 & 22.2 & 27.3 & 88.9 \\
\hline Pensions & 21.1 & 4.1 & 4.9 & 4.9 & 0.8 & 1.3 & 1.2 & 1.6 & 5.0 \\
\hline Subsidies & 58.9 & 38.4 & 20.5 & 20.5 & 3.7 & 5.8 & 6.4 & 4.6 & 20.5 \\
\hline Of which: gas subsidy & 37.4 & 16.4 & 0.0 & 0.0 & 0.0 & 0.0 & 0.0 & 0.0 & 0.0 \\
\hline Interest & 10.0 & 10.4 & 23.3 & 15.9 & 3.5 & 3.1 & 7.5 & 9.2 & 33.1 \\
\hline Social allowances and pensions & 183.7 & 206.2 & 243.1 & 243.1 & 55.7 & 61.2 & 61.0 & 65.2 & 245.6 \\
\hline Of which: social insurance & 85.8 & 153.5 & 178.8 & 178.8 & 43.2 & 45.3 & 45.2 & 45.1 & 180.6 \\
\hline Goods and services & 206.7 & 133.1 & 165.7 & 168.9 & 27.6 & 42.2 & 41.8 & 54.1 & 163.9 \\
\hline Grants & $\ldots$ & 52.9 & 66.2 & 66.2 & 13.1 & 16.2 & 17.6 & 19.3 & 59.0 \\
\hline Other expenditure & $\ldots$ & 133.9 & 120.2 & 163.6 & 22.6 & 30.7 & 33.0 & 34.0 & 120.2 \\
\hline Transactions in non-financial assets & 164.5 & 141.5 & 166.6 & 174.7 & 16.0 & 62.6 & 54.5 & 33.5 & 180.5 \\
\hline Acquisition of non-financial assets & 183.3 & 162.6 & 179.9 & 188.0 & 17.8 & 67.2 & 59.0 & 35.9 & 193.5 \\
\hline Of which: projects related to the Russian loan & & $\ldots$ & 24.0 & & 0.0 & 2.0 & 13.2 & 8.8 & 53.0 \\
\hline Disposals of non-financial assets & 18.9 & 21.1 & 13.3 & 13.3 & 1.8 & 4.6 & 4.5 & 2.4 & 13.0 \\
\hline Overall balance (above-the-line) & -73.5 & -62.7 & -217.0 & -40.0 & -23.2 & -73.8 & -62.2 & -57.8 & -203.7 \\
\hline Statistical discrepancy & 4.1 & 16.1 & 0.0 & 0.0 & -3.2 & 1.1 & 1.1 & 1.1 & 0.0 \\
\hline Overall balance (below-the-line) & -69.4 & -46.6 & -217.0 & -40.0 & -26.4 & -72.7 & -61.2 & -56.7 & -203.7 \\
\hline Financing & 69.4 & 46.6 & 217.0 & 40.0 & 26.4 & 72.7 & 61.2 & 56.7 & 203.7 \\
\hline Domestic financing & 30.6 & 28.6 & -113.8 & 30.7 & 24.1 & -210.3 & 37.7 & 34.7 & 97.5 \\
\hline Banking system & -6.4 & -4.7 & -79.3 & 9.5 & 28.4 & -207.0 & 53.9 & 45.4 & 142.3 \\
\hline CBA & -16.2 & -28.1 & -111.3 & 9.5 & 37.8 & -220.3 & 36.8 & 34.4 & 117.3 \\
\hline Of which: deposits related to the Russian loan & 0.0 & 0.0 & -134.8 & & 0.0 & -186.5 & 31.1 & 20.7 & 96.3 \\
\hline Commercial Banks & 9.9 & 23.4 & 32.0 & 0.0 & -9.4 & 13.3 & 17.2 & 11.0 & 25.0 \\
\hline Nonbanks & 37.0 & 33.4 & -34.6 & 21.2 & -4.3 & -3.3 & -16.3 & -10.7 & -44.9 \\
\hline Privatization proceeds & 44.2 & 31.6 & 0.0 & 0.0 & 0.0 & 0.0 & 0.0 & 0.0 & 0.0 \\
\hline T-Bills & -2.8 & 3.5 & 3.0 & 25.0 & -1.3 & 1.8 & 1.8 & 0.7 & 2.3 \\
\hline Promissory note/other & -1.0 & -1.2 & -0.1 & -0.1 & 0.0 & 0.0 & 0.0 & -0.1 & 0.0 \\
\hline Net lending & -3.4 & -0.5 & -37.5 & -3.7 & -3.0 & -5.1 & -18.0 & -11.4 & -47.2 \\
\hline Of which: projects financed with the Russian loan & $\cdots \cdot$ & $\cdots$ & -33.8 & $\ldots$ & 0.0 & -4.0 & -17.9 & -11.9 & -43.3 \\
\hline External financing & 38.8 & 17.9 & 330.9 & 9.3 & 2.3 & 283.1 & 23.5 & 22.0 & 106.3 \\
\hline Gross inflow & 64.5 & 48.8 & 370.8 & 49.2 & 12.8 & 292.7 & 35.5 & 29.9 & 148.6 \\
\hline Of which: Russian project loan & & & 192.5 & & 0.0 & 192.5 & 0.0 & 0.0 & 0.0 \\
\hline Amortization due & -6.0 & -4.9 & -6.6 & -6.6 & -3.3 & -0.6 & -2.3 & -0.5 & -7.1 \\
\hline Net lending & -19.7 & -26.0 & -33.3 & -33.3 & -7.2 & -9.0 & -9.7 & -7.4 & -35.2 \\
\hline \multicolumn{10}{|l|}{ Memorandum items: } \\
\hline Nominal GDP (billion of drams) & 3,149 & 3,646 & 3,350 & 4,169 & 3,350 & 3,350 & 3,350 & 3,350 & 3,487 \\
\hline Underlying balance $2 /$ & -89.5 & -58.1 & -249.2 & -70.7 & -30.7 & -83.4 & -71.2 & -63.8 & -222.4 \\
\hline Program balance 3 / & -92.5 & -73.1 & -287.8 & $\ldots$ & -36.6 & -86.8 & -88.9 & -75.4 & -286.1 \\
\hline Overall balance excl. spending financed with the Russian loan & $\ldots$ & $\ldots$ & -193.0 & $\ldots$ & -23.2 & -71.8 & -49.0 & -49.0 & -150.7 \\
\hline
\end{tabular}


Table 5. Armenia: Central Government Operations, 2007-10 (concluded)

(in percent of GDP, unless otherwise specified)

\begin{tabular}{|c|c|c|c|c|c|c|c|c|c|}
\hline & \multirow{3}{*}{$\frac{\frac{2007}{\text { Jan.-Dec. }}}{\text { Act. }}$} & \multirow{3}{*}{$\frac{2008}{\frac{\text { Jan.-Dec. }}{\text { Prelim. }}}$} & \multirow{2}{*}{\multicolumn{2}{|c|}{$\begin{array}{c}2009 \\
\text { Jan.-Dec. }\end{array}$}} & \multicolumn{4}{|c|}{2009} & \multirow{3}{*}{$\frac{2010}{\text { Jan.-Dec. }}$} \\
\hline & & & & & \multirow{2}{*}{$\begin{array}{c}\text { Q1 } \\
\text { Prel. }\end{array}$} & \multirow{2}{*}{$\begin{array}{c}\text { Q2 } \\
\text { Proj. }\end{array}$} & \multirow{2}{*}{$\begin{array}{l}\text { Q3 } \\
\text { Proj. }\end{array}$} & \multirow{2}{*}{$\begin{array}{c}\text { Q4 } \\
\text { Proj. }\end{array}$} & \\
\hline & & & IMF Proj. & Budget & & & & & \\
\hline Total revenue and grants & 20.1 & 20.0 & 20.4 & 21.1 & 4.0 & 5.2 & 5.5 & 5.7 & 20.4 \\
\hline Total revenue & 19.3 & 19.6 & 19.1 & 20.3 & 3.9 & 4.8 & 5.1 & 5.3 & 19.4 \\
\hline Tax revenues & 16.0 & 16.4 & 15.6 & 16.8 & 3.1 & 4.0 & 4.2 & 4.4 & 16.0 \\
\hline VAT & 7.9 & 8.1 & 7.5 & $\ldots$ & 1.4 & 1.9 & 2.0 & 2.2 & $\ldots$ \\
\hline Profits, simplified and presumptive & 3.3 & 3.1 & 2.9 & $\ldots$ & 0.6 & 0.8 & 0.7 & 0.7 & $\ldots$ \\
\hline Personal income tax & 1.5 & 1.5 & 1.5 & $\ldots$ & 0.4 & 0.4 & 0.4 & 0.4 & $\ldots$ \\
\hline Customs duties & 0.8 & 1.0 & 0.8 & $\ldots$ & 0.2 & 0.2 & 0.2 & 0.2 & $\ldots$ \\
\hline Other & 2.6 & 2.7 & 2.9 & $\ldots$ & 0.5 & 0.8 & 0.9 & 0.8 & $\ldots$ \\
\hline Social contributions & 2.7 & 2.9 & 3.0 & 3.1 & 0.6 & 0.7 & 0.7 & 0.9 & 3.1 \\
\hline Other revenue & 0.6 & 0.4 & 0.4 & 0.4 & 0.2 & 0.1 & 0.1 & 0.0 & 0.3 \\
\hline Grants & 0.7 & 0.4 & 1.3 & 0.8 & 0.2 & 0.4 & 0.4 & 0.4 & 1.0 \\
\hline Total expenditure & 22.4 & 21.8 & 26.9 & 22.1 & 4.7 & 7.4 & 7.3 & 7.4 & 26.3 \\
\hline Expense & 17.2 & 17.9 & 21.9 & 17.9 & 4.2 & 5.5 & 5.7 & 6.4 & 21.1 \\
\hline Wages 1/ & 1.9 & 2.0 & 2.7 & 1.5 & 0.4 & 0.7 & 0.7 & 0.8 & $\ldots$ \\
\hline Pensions & 0.7 & 0.1 & 0.1 & 0.1 & 0.0 & 0.0 & 0.0 & 0.0 & $\ldots$ \\
\hline Subsidies & 1.9 & 1.1 & 0.6 & 0.5 & 0.1 & 0.2 & 0.2 & 0.1 & $\ldots$ \\
\hline Of which: gas subsidy & 1.2 & 0.5 & 0.0 & 0.0 & 0.0 & 0.0 & 0.0 & 0.0 & 0.0 \\
\hline Interest & 0.3 & 0.3 & 0.7 & 0.4 & 0.1 & 0.1 & 0.2 & 0.3 & 0.9 \\
\hline Social allowances and pensions & 5.8 & 5.7 & 7.3 & 5.8 & 1.7 & 1.8 & 1.8 & 1.9 & 7.0 \\
\hline Of which: social insurance & 2.7 & 4.2 & 5.3 & 4.3 & 1.3 & 1.4 & 1.3 & 1.3 & 5.2 \\
\hline Goods and services & 6.6 & 3.7 & 4.9 & 4.1 & 0.8 & 1.3 & 1.2 & 1.6 & $\ldots$ \\
\hline Grants & $\ldots$ & 1.5 & 2.0 & 1.6 & 0.4 & 0.5 & 0.5 & 0.6 & $\ldots$ \\
\hline Other expenditure & $\ldots$ & 3.7 & 3.6 & 3.9 & 0.7 & 0.9 & 1.0 & 1.0 & $\ldots$ \\
\hline Transactions in non-financial assets & 5.2 & 3.9 & 5.0 & 4.2 & 0.5 & 1.9 & 1.6 & 1.0 & 5.2 \\
\hline Acquisition of non-financial assets & 5.8 & 4.5 & 5.4 & 4.5 & 0.5 & 2.0 & 1.8 & 1.1 & 5.5 \\
\hline of which: projects related to the Russian loan & $\ldots$ & & 0.7 & & 0.0 & 0.1 & 0.4 & 0.3 & 1.5 \\
\hline Disposals of non-financial assets & 0.6 & 0.6 & 0.4 & 0.3 & 0.1 & 0.1 & 0.1 & 0.1 & 0.4 \\
\hline Overall balance (above-the-line) & -2.3 & -1.7 & -6.5 & -1.0 & -0.7 & -2.2 & -1.9 & -1.7 & -5.8 \\
\hline Statistical discrepancy & 0.1 & 0.4 & 0.0 & 0.0 & -0.1 & 0.0 & 0.0 & 0.0 & 0.0 \\
\hline Overall balance (below-the-line) & -2.2 & -1.3 & -6.5 & -1.0 & -0.8 & -2.2 & -1.8 & -1.7 & -5.8 \\
\hline Financing & 2.2 & 1.3 & 6.5 & 1.0 & 0.8 & 2.2 & 1.8 & 1.7 & 5.8 \\
\hline Domestic financing & 1.0 & 0.8 & -3.4 & 0.7 & 0.7 & -6.3 & 1.1 & 1.0 & 2.8 \\
\hline Banking system & -0.2 & -0.1 & -2.4 & 0.2 & 0.8 & -6.2 & 1.6 & 1.4 & 4.1 \\
\hline CBA & -0.5 & -0.8 & -3.3 & $\ldots$ & 1.1 & -6.6 & 1.1 & 1.0 & 3.4 \\
\hline of which: deposits related to the Russian loan & $\ldots$ & & -4.0 & $\ldots$ & 0.0 & -5.6 & 0.9 & 0.6 & 2.8 \\
\hline Commercial Banks & 0.3 & 0.6 & 1.0 & $\ldots$ & -0.3 & 0.4 & 0.5 & 0.3 & 0.7 \\
\hline Nonbanks & 1.2 & 0.9 & -1.0 & 0.5 & -0.1 & -0.1 & -0.5 & -0.3 & -1.3 \\
\hline Privatization proceeds & 1.4 & 0.9 & 0.0 & 0.0 & 0.0 & 0.0 & 0.0 & 0.0 & 0.0 \\
\hline T-Bills & -0.1 & 0.1 & 0.1 & 0.6 & 0.0 & 0.1 & 0.1 & 0.0 & 0.1 \\
\hline Promissory note/other & 0.0 & 0.0 & 0.0 & 0.0 & 0.0 & 0.0 & 0.0 & 0.0 & 0.0 \\
\hline Net lending & -0.1 & 0.0 & -1.1 & -0.1 & -0.1 & -0.2 & -0.5 & -0.3 & -1.4 \\
\hline of which: projects financed with the Russian loan & $\cdots$ & $\ldots$ & -1.0 & $\ldots$ & 0.0 & -0.1 & -0.5 & -0.4 & -1.2 \\
\hline External financing & 1.2 & 0.5 & 9.9 & 0.2 & 0.1 & 8.5 & 0.7 & 0.7 & 3.0 \\
\hline Gross inflow & 2.0 & 1.3 & 11.1 & 1.2 & 0.4 & 8.7 & 1.1 & 0.9 & 4.3 \\
\hline of which: Russian project loan & & & 5.7 & & 0.0 & 5.7 & 0.0 & 0.0 & 0.0 \\
\hline Amortization due & -0.2 & -0.1 & -0.2 & -0.2 & -0.1 & 0.0 & -0.1 & 0.0 & -0.2 \\
\hline Net lending & -0.6 & -0.7 & -1.0 & -0.8 & -0.2 & -0.3 & -0.3 & -0.2 & -1.0 \\
\hline \multicolumn{10}{|l|}{ Memorandum items } \\
\hline Nominal GDP (billion of drams) & 3,149 & 3,646 & 3,350 & 4,169 & 3,350 & 3,350 & 3,350 & 3,350 & 3,487 \\
\hline Underlying balance $2 /$ & -2.8 & -1.6 & -7.4 & -0.9 & -0.9 & -2.5 & -2.1 & -1.9 & -6.4 \\
\hline Program balance $3 /$ & -2.9 & -2.0 & -8.6 & $\ldots$ & -1.1 & -2.6 & -2.7 & -2.3 & -8.2 \\
\hline Overall balance excl. spending financed with the Russian loan & $\ldots$ & $\ldots$ & -5.8 & $\ldots$ & -0.7 & -2.1 & -1.5 & -1.5 & -4.3 \\
\hline
\end{tabular}

Sources: Ministry of Finance and Economy, Central Bank of Armenia, and Fund staff estimates and projections.

$1 /$ Relative to the budget, the staff presentation reclassifies estimated military wages from "Goods and services" and "Other expenditure" to "Wages". 2/ Overall balance before grants, and excluding external interest payments.

$3 /$ The program balance reflects net lending activities and is measured as below-the-line overall balance minus net lending. 
Table 6. Armenia: Public Sector Debt Sustainability Framework, 2004-14

(In percent of GDP, unless otherwise indicated)

\begin{tabular}{|c|c|c|c|c|c|c|c|c|c|c|c|c|}
\hline & \multicolumn{5}{|c|}{ Actual } & \multicolumn{6}{|c|}{ Projections } & \multirow{3}{*}{$\begin{array}{c}\text { Debt-stabilizing } \\
\text { primary } \\
\text { balance } 9 /\end{array}$} \\
\hline & 2004 & 2005 & 2006 & 2007 & 2008 & 2009 & 2010 & 2011 & 2012 & 2013 & 2014 & \\
\hline & & & & & & & & & & & & \\
\hline Baseline: Public sector debt $1 /$ & 32.4 & 24.3 & 18.7 & 17.5 & 17.8 & 37.8 & 43.7 & 45.8 & 42.2 & 39.7 & 36.1 & -2.1 \\
\hline Of which: foreign-currency denominated & 30.1 & 22.1 & 16.5 & 15.4 & 15.3 & 34.0 & 39.2 & 40.3 & 35.9 & 32.2 & 27.6 & \\
\hline Change in public sector debt & -8.5 & -8.1 & -5.6 & -1.2 & 0.4 & 20.0 & 5.8 & 2.1 & -3.6 & -2.4 & -3.7 & \\
\hline Identified debt-creating flows $(4+7+12)$ & -8.0 & -5.3 & -5.3 & -2.5 & -0.6 & 5.9 & 3.6 & 1.2 & 0.1 & 0.4 & -0.7 & \\
\hline Primary deficit & 1.2 & 1.0 & 1.1 & 1.3 & 0.7 & 3.7 & 3.8 & 2.4 & 2.3 & 2.3 & 1.1 & \\
\hline Revenue and grants & 15.4 & 17.8 & 18.0 & 20.1 & 20.0 & 20.4 & 20.4 & 20.8 & 21.3 & 21.1 & 21.4 & \\
\hline Primary (noninterest) expenditure & 16.6 & 18.8 & 19.0 & 21.4 & 20.8 & 24.0 & 24.2 & 23.3 & 23.5 & 23.4 & 22.5 & \\
\hline Automatic debt dynamics $2 /$ & -9.2 & -6.3 & -6.8 & -4.7 & -2.6 & 2.2 & -0.2 & -1.2 & -2.2 & -2.0 & -1.8 & \\
\hline Contribution from interest rate/growth differential 3/ & -4.4 & -4.4 & -3.1 & -2.3 & -1.1 & 2.2 & -0.2 & -1.2 & -2.2 & -2.0 & -1.8 & \\
\hline Of which: contribution from real interest rate & -0.6 & -0.6 & -0.4 & -0.1 & 0.0 & 0.4 & 0.3 & 0.2 & -0.2 & -0.2 & -0.1 & \\
\hline Of which: contribution from real GDP growth & -3.8 & -3.8 & -2.7 & -2.2 & -1.1 & 1.8 & -0.5 & -1.4 & -1.9 & -1.8 & -1.7 & \\
\hline Contribution from exchange rate depreciation $4 /$ & -4.8 & -1.9 & -3.7 & -2.3 & -1.5 & 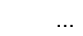 & $\ldots$ & $\ldots$ & $\ldots$ & $\ldots$ & $\ldots$ & \\
\hline Other identified debt-creating flows & 0.0 & 0.0 & 0.5 & 0.9 & 1.2 & 0.0 & 0.0 & 0.0 & 0.0 & 0.0 & 0.0 & \\
\hline Privatization receipts (negative) & 0.0 & 0.0 & 0.0 & 0.0 & 0.0 & 0.0 & 0.0 & 0.0 & 0.0 & 0.0 & 0.0 & \\
\hline Recognition of implicit or contingent liabilities & 0.0 & 0.0 & 0.0 & 0.0 & 0.0 & 0.0 & 0.0 & 0.0 & 0.0 & 0.0 & 0.0 & \\
\hline Other (specify, e.g. bank recapitalization) & 0.0 & 0.0 & 0.5 & 0.9 & 1.2 & 0.0 & 0.0 & 0.0 & 0.0 & 0.0 & 0.0 & \\
\hline Residual, including asset changes (2-3) $5 /$ & -0.6 & -2.8 & -0.4 & 1.2 & 1.0 & 14.2 & 2.3 & 0.9 & -3.7 & -2.8 & -2.9 & \\
\hline Public sector debt-to-revenue ratio $1 /$ & 210.4 & 136.4 & 104.1 & 86.9 & 88.9 & 185.8 & 213.5 & 219.6 & 198.3 & 188.6 & 168.4 & \\
\hline Gross financing need $6 /$ & 7.4 & 7.0 & 6.3 & 5.4 & 4.1 & 10.7 & 11.1 & 9.2 & 10.7 & 12.1 & 11.9 & \\
\hline in billions of U.S. dollars & 0.3 & 0.3 & 0.4 & 0.5 & 0.5 & 1.0 & 1.0 & 0.9 & 1.1 & 1.3 & 1.4 & \\
\hline Scenario with key variables at their historical averages 71 & & & & & & 37.8 & 38.1 & 37.0 & 32.1 & 28.6 & 25.4 & -2.7 \\
\hline Scenario with no policy change (constant primary balance) in 2009-2014 & & & & & & 37.8 & 43.6 & 47.0 & 44.7 & 43.6 & 42.4 & -2.4 \\
\hline \multicolumn{13}{|l|}{ Key Macroeconomic and Fiscal Assumptions Underlying Baseline } \\
\hline Real GDP growth (in percent) & 10.5 & 13.9 & 13.2 & 13.7 & 6.8 & -9.5 & 1.3 & 3.5 & 4.5 & 4.5 & 4.5 & \\
\hline Average nominal interest rate on public debt (in percent) $8 /$ & 1.5 & 1.7 & 1.7 & 2.0 & 1.9 & 3.6 & 2.6 & 2.4 & 1.4 & 1.5 & 1.6 & \\
\hline Average real interest rate (nominal rate minus change in GDP deflator, in percent) & -1.4 & -1.6 & -1.5 & -0.5 & 0.3 & 1.7 & 0.7 & 0.6 & -0.5 & -0.4 & -0.3 & \\
\hline Nominal appreciation (increase in U.S. dollar value of local currency, in percent) & 16.5 & 7.9 & 23.8 & 19.5 & 11.8 & & $\ldots$ & $\ldots$ & & & $\ldots$ & \\
\hline Inflation rate (GDP deflator, in percent) & 2.9 & 3.2 & 3.2 & 2.6 & 1.6 & 1.9 & 1.9 & 1.9 & 1.9 & 1.9 & 1.9 & \\
\hline Growth of real primary spending (deflated by GDP deflator, in percent) & 4.3 & 29.0 & 16.2 & 29.8 & 10.7 & 4.4 & 2.9 & 1.1 & 8.0 & 6.0 & 2.1 & \\
\hline Primary deficit & 1.2 & 1.0 & 1.1 & 1.3 & 0.7 & 3.7 & 3.8 & 2.4 & 2.3 & 2.3 & 1.1 & \\
\hline
\end{tabular}

$1 /$ Indicate coverage of public sector, e.g., general government or nonfinancial public sector. Also whether net or gross debt is used.

2/ Derived as [(r- $\pi(1+g)-g+\alpha \varepsilon(1+r)] /(1+g+\pi+g \pi))$ times previous period debt ratio, with $r=$ interest rate; $\pi=$ growth rate of GDP deflator; $g=$ real GDP growth rate; $\alpha=$ share of foreign-currency denominated debt; and $\varepsilon=$ nominal exchange rate depreciation (measured by increase in local currency value of U.S. dollar).

$3 /$ The real interest rate contribution is derived from the denominator in footnote $2 /$ as $r-\pi(1+g)$ and the real growth contribution as $-g$.

$4 /$ The exchange rate contribution is derived from the numerator in footnote $2 /$ as $\alpha \varepsilon(1+r)$.

$6 /$ Defined as public sector deficit, plus amortization of medium and long-term public sector debt, plus short-term debt at end of previous period.

7/ The key variables include real GDP growth; real interest rate; and primary balance in percent of GDP.

8/ Derived as nominal interest expenditure divided by previous period debt stock.

9/ Assumes that key variables (real GDP growth, real interest rate, and other identified debt-creating flows) remain at the level of the last projection year. 
Table 7. Armenia: External Debt Sustainability Framework, 2004-2014

(In percent of GDP, unless otherwise indicated)

\begin{tabular}{|c|c|c|c|c|c|c|c|c|c|c|c|c|}
\hline & \multicolumn{5}{|c|}{ Actual } & \multicolumn{7}{|c|}{ Projections } \\
\hline & 2004 & 2005 & 2006 & 2007 & 2008 & 2009 & 2010 & 2011 & 2012 & 2013 & 2014 & \multirow{3}{*}{$\begin{array}{c}\text { Debt-stabilizing } \\
\text { non-interest } \\
\text { current account } 6 / \\
-8.6\end{array}$} \\
\hline & & & & & & & & & & & & \\
\hline Baseline: External debt & 33.3 & 22.4 & 18.9 & 17.3 & 15.3 & 31.7 & 38.9 & 39.5 & 36.1 & 32.1 & 28.2 & \\
\hline Change in external debt & -5.8 & -10.9 & -3.5 & -1.6 & -2.0 & 16.5 & 7.2 & 0.6 & -3.4 & -4.0 & -3.8 & \\
\hline Identified external debt-creating flows $(4+8+9)$ & -8.6 & -12.3 & -9.9 & -6.7 & 0.3 & 10.2 & 7.6 & 3.8 & 0.4 & -2.9 & -3.2 & \\
\hline Current account deficit, excluding interest payments & 4.3 & 0.8 & 1.7 & 6.3 & 11.2 & 12.7 & 12.9 & 10.5 & 7.9 & 5.5 & 4.6 & \\
\hline Deficit in balance of goods and services & 14.9 & 14.4 & 16.1 & 19.7 & 24.8 & 24.0 & 25.2 & 24.8 & 23.6 & 22.4 & 20.9 & \\
\hline Exports & 27.7 & 28.9 & 23.7 & 19.3 & 14.5 & 13.6 & 16.5 & 18.3 & 19.6 & 20.4 & 21.0 & \\
\hline Imports & 42.6 & 43.3 & 39.7 & 39.0 & 39.3 & 37.7 & 41.7 & 43.1 & 43.2 & 42.8 & 41.9 & \\
\hline Net non-debt creating capital inflows (negative) & -6.1 & -4.7 & -7.0 & -7.6 & -7.9 & -4.7 & -5.5 & -6.1 & -6.4 & -7.4 & -7.0 & \\
\hline Automatic debt dynamics $1 /$ & -6.8 & -8.4 & -4.6 & -5.4 & -3.0 & 2.2 & 0.2 & -0.7 & -1.1 & -1.0 & -0.9 & \\
\hline Contribution from nominal interest rate & 0.3 & 0.2 & 0.2 & 0.1 & 0.1 & 0.3 & 0.6 & 0.7 & 0.6 & 0.6 & 0.5 & \\
\hline Contribution from real GDP growth & -3.4 & -3.4 & -2.3 & -1.8 & -1.0 & 1.9 & -0.4 & -1.3 & -1.7 & -1.5 & -1.3 & \\
\hline Contribution from price and exchange rate changes $2 /$ & -3.7 & -5.2 & -2.4 & -3.7 & -2.2 & $\ldots$ & ... & $\ldots$ & .. & $\ldots$ & ... & \\
\hline Residual, incl. change in gross foreign assets $(2-3) 3 /$ & 2.7 & 1.4 & 6.4 & 5.1 & -2.3 & 6.3 & -0.4 & -3.2 & -3.8 & -1.1 & -0.6 & \\
\hline External debt-to-exports ratio (in percent) & 120.1 & 77.6 & 79.8 & 89.6 & 105.5 & 232.7 & 235.2 & 215.7 & 184.1 & 157.2 & 134.3 & \\
\hline Gross external financing need (in billions of US dollars) $4 /$ & 0.2 & 0.1 & 0.2 & 0.6 & 1.4 & 1.3 & 1.3 & 1.1 & 1.0 & 1.0 & 0.9 & \\
\hline in percent of GDP & 7.0 & 2.1 & 2.6 & 6.8 & 11.6 & 13.6 & 14.3 & 12.2 & 11.1 & 10.0 & 8.4 & \\
\hline Scenario with key variables at their historical averages $5 /$ & & & & & & 31.7 & 26.6 & 21.7 & 17.8 & 16.5 & 16.1 & -8.1 \\
\hline \multicolumn{13}{|l|}{ Key Macroeconomic Assumptions Underlying Baseline } \\
\hline Real GDP growth (in percent) & 10.5 & 13.9 & 13.2 & 13.7 & 6.8 & -9.5 & 1.3 & 3.5 & 4.5 & 4.5 & 4.5 & \\
\hline GDP deflator in US dollars (change in percent) & 10.4 & 18.6 & 12.0 & 24.0 & 14.5 & -13.9 & -6.7 & -1.7 & 0.1 & 1.7 & 3.3 & \\
\hline Nominal external interest rate (in percent) & 0.9 & 1.0 & 0.9 & 0.9 & 0.8 & 1.8 & 1.8 & 1.7 & 1.7 & 1.7 & 1.6 & \\
\hline Growth of exports (US dollar terms, in percent) & 9.0 & 43.8 & 6.7 & 17.6 & -3.0 & -26.2 & 16.7 & 15.4 & 15.0 & 13.2 & 13.3 & \\
\hline Growth of imports (US dollar terms, in percent) & 7.7 & 40.3 & 19.4 & 41.5 & 30.3 & -24.8 & 6.5 & 7.6 & 7.7 & 7.9 & 7.5 & \\
\hline Current account balance, excluding interest payments & -4.3 & -0.8 & -1.7 & -6.3 & -11.2 & -12.7 & -12.9 & -10.5 & -7.9 & -5.5 & -4.6 & \\
\hline Net non-debt creating capital inflows & 6.1 & 4.7 & 7.0 & 7.6 & 7.9 & 4.7 & 5.5 & 6.1 & 6.4 & 7.4 & 7.0 & \\
\hline
\end{tabular}

$1 /$ Derived as $[r-g-\rho(1+g)+\varepsilon \alpha(1+r)](1+g+\rho+g \rho)$ times previous period debt stock, with $r=$ nominal effective interest rate on external debt; $\rho=$ change in domestic GDP deflator in US dollar terms, $g=$ real GDP growth $\varepsilon=$ nominal appreciation (increase in dollar value of domestic currency), and $\alpha=$ share of domestic-currency denominated debt in total external debt.

$2 /$ The contribution from price and exchange rate changes is defined as $[-\rho(1+g)+\varepsilon \alpha(1+r)] /(1+g+\rho+g \rho)$ times previous period debt stock. $\rho$ increases with an appreciating domestic currency $(\varepsilon>0)$ and rising inflation $(b a$ $3 /$ For projection, line includes the impact of price and exchange rate changes.

4/ Defined as current account deficit, plus amortization on medium- and long-term debt, plus short-term debt at end of previous period.

5/ The key variables include real GDP growth; nominal interest rate; dollar deflator growth; and both non-interest current account and non-debt inflows in percent of GDP.

6/ Long-run, constant balance that stabilizes the debt ratio assuming that key variables (real GDP growth, nominal interest rate, dollar deflator growth, and non-debt inflows in percent of GDP) remain at their levels

of the last projection year. 
Table 8. Armenia: Medium-Term Macroeconomic Framework, 2007-14

\begin{tabular}{|c|c|c|c|c|c|c|c|c|}
\hline & 2007 & 2008 & 2009 & 2010 & 2011 & 2012 & 2013 & 2014 \\
\hline & & Prel. & \multicolumn{6}{|c|}{ Projections } \\
\hline & \multicolumn{8}{|c|}{ (In percent of GDP, unless otherwise specified) } \\
\hline \multicolumn{9}{|l|}{ National income and prices } \\
\hline Real GDP (percent change) & 13.7 & 6.8 & -9.5 & 1.3 & 3.5 & 4.5 & 4.5 & 4.5 \\
\hline Gross domestic product (in millions of U.S. dollars) & 9,206 & 11,917 & 9,333 & 8,980 & 9,360 & 10,049 & 10,940 & 12,026 \\
\hline Gross national income per capita (in U.S. dollars) & 2,940 & 3,830 & 2,937 & 2,806 & 2,907 & 3,099 & 3,353 & 3,649 \\
\hline \multicolumn{9}{|l|}{ Investment and saving } \\
\hline Investment & 38.6 & 41.7 & 35.8 & 36.5 & 37.0 & 37.7 & 38.3 & 38.8 \\
\hline Private & 33.4 & 37.8 & 30.8 & 31.3 & 31.8 & 32.3 & 32.8 & 33.3 \\
\hline Public & 5.2 & 3.9 & 5.0 & 5.2 & 5.2 & 5.4 & 5.5 & 5.5 \\
\hline National savings & 32.2 & 30.4 & 22.7 & 23.0 & 25.8 & 29.2 & 32.3 & 33.8 \\
\hline Private & 29.3 & 28.2 & 24.3 & 23.7 & 25.3 & 27.8 & 30.4 & 30.9 \\
\hline Public & 2.9 & 2.2 & -1.5 & -0.7 & 0.5 & 1.5 & 2.0 & 2.9 \\
\hline \multicolumn{9}{|l|}{ Central government operations } \\
\hline Revenue and grants & 20.1 & 20.0 & 20.4 & 20.4 & 20.8 & 21.3 & 21.1 & 21.4 \\
\hline Of which: tax revenue & 16.0 & 16.4 & 15.6 & 16.0 & 16.4 & 16.8 & 17.2 & 17.6 \\
\hline grants 1/ & 0.7 & 0.4 & 1.3 & 1.0 & 1.0 & 1.0 & 0.4 & 0.4 \\
\hline Expenditure & 22.4 & 21.8 & 26.9 & 26.3 & 25.5 & 25.3 & 24.7 & 24.1 \\
\hline Current expenditure & 17.2 & 17.9 & 21.9 & 21.1 & 20.3 & 19.8 & 19.1 & 18.5 \\
\hline Capital expenditure & 5.2 & 3.9 & 5.0 & 5.2 & 5.2 & 5.4 & 5.7 & 5.7 \\
\hline Overall balance (including grants) & -2.2 & -1.3 & -6.5 & -5.8 & -4.6 & -4.0 & -3.6 & -2.6 \\
\hline Domestic financing & 1.0 & 0.8 & -3.4 & 2.8 & 2.8 & 2.4 & 3.4 & 2.4 \\
\hline External financing & 1.2 & 0.5 & 9.9 & 3.0 & 1.8 & 1.6 & 0.2 & 0.2 \\
\hline Underlying balance $2 /$ & -2.8 & -1.6 & -7.4 & -6.4 & -5.1 & -4.5 & -3.5 & -2.6 \\
\hline Government and government-guaranteed debt & 17.5 & 17.8 & 37.8 & 43.7 & 45.8 & 42.2 & 39.7 & 36.1 \\
\hline \multicolumn{9}{|l|}{ External sector } \\
\hline Exports of goods and services & 19.3 & 14.5 & 13.6 & 16.5 & 18.3 & 19.6 & 20.4 & 21.0 \\
\hline Imports of goods and services & 39.0 & 39.3 & 37.7 & 41.7 & 43.1 & 43.2 & 42.8 & 41.9 \\
\hline Current account (in percent of GDP) & -6.4 & -11.3 & -13.0 & -13.5 & -11.2 & -8.5 & -6.0 & -5.0 \\
\hline Of which: direct foreign investment & 701 & 936 & 440 & 490 & 568 & 648 & 812 & 836 \\
\hline public sector disbursements & 149 & 160 & 963 & 383 & 202 & 194 & 205 & 185 \\
\hline Change in gross international reserves (in millions of U.S. dollars) $3 /$ & -587 & 254 & -200 & 58 & 100 & 11 & -220 & -252 \\
\hline Arrears and debt relief (in millions of U.S. dollars) & 1 & 0 & 2 & 2 & 2 & 2 & 2 & 2 \\
\hline Financing/gap (in millions of U.S. dollars) & 0 & 0 & 218 & 440 & 66 & 0 & 0 & 0 \\
\hline Of which: IMF & 0 & 0 & 112 & 139 & 56 & 0 & 0 & 0 \\
\hline Other & 0 & 0 & 0 & 149 & 10 & 0 & 0 & 0 \\
\hline Gross international reserves in months of imports & 4.3 & 4.8 & 5.1 & 4.6 & 4.0 & 3.7 & 3.9 & 4.1 \\
\hline
\end{tabular}

Sources: Armenian authorities; and Fund staff estimates and projections.

1/ For 2007 -09, the figures include projections for disbursements under the U.S. Millennium Challenge Account. 2/ Underlying balance is defined as overall balance before grants and excluding external interest payments. 3/ A negative figure indicates an increase. 
Table 9. Armenia: Fund Disbursements and Timing of Reviews Under the Twenty Eight -Month Stand-By Arrangement, 2009-11 (SDR millions)

\begin{tabular}{|c|c|c|c|c|}
\hline Date of Availability & Conditions & SBA & Augmentation & Total \\
\hline 6 March, 2009 & Board approval of the Stand -By arrangement & 161.552 & & 161.552 \\
\hline 15 May, 2009 & Observance of end -March 2009 performance criteria and completion of first review & 36.800 & & 36.800 \\
\hline 22 June, 2009 & Board approval of the SBA augmentation & & 65.868 & 65.868 \\
\hline 15 August, 2009 & Observance of end -June 2009 performance criteria and completion of second review & 18.860 & & 18.860 \\
\hline 15 November, 2009 & Observance of end -September 2009 performance criteria and completion of third review & 18.860 & & 18.860 \\
\hline 15 February, 2010 & Observance of end -December 2009 performance criteria and completion of fourth review & 23.552 & 24.933 & 48.485 \\
\hline 15 May, 2010 & Observance of end -March 2010 performance criteria and completion of fifth review & 23.552 & 24.933 & 48.485 \\
\hline 15 August, 2010 & Observance of end -June 2010 performance criteria and completion of sixth review & 23.552 & 24.933 & 48.485 \\
\hline 15 November, 2010 & Observance of end -September 2010 performance criteria and completion of seventh review & 23.552 & 24.933 & 48.485 \\
\hline 15 February, 2011 & Observance of end -December 2010 performance criteria and completion of eighth review & 18.860 & & 18.860 \\
\hline 15 May, 2011 & Observance of end -March 2011 performance criteria and completion of ninth review & 18.860 & & 18.860 \\
\hline
\end{tabular}

Source: Fund staff estimates and projections. 
Table 10. Armenia: Indicators of Capacity to Repay the Fund, 2008-15

\begin{tabular}{|c|c|c|c|c|c|c|c|c|}
\hline & 2008 & 2009 & 2010 & 2011 & 2012 & 2013 & 2014 & 2015 \\
\hline & Prelim. & \multicolumn{7}{|c|}{ Projections } \\
\hline \multicolumn{9}{|l|}{$\begin{array}{l}\text { Fund obligations based on existing credit } \\
\text { (in millions of SDRs) }\end{array}$} \\
\hline Principal & 17.1 & 15.2 & 14.1 & 14.4 & 75.3 & 91.9 & 27.9 & 4.5 \\
\hline Charges and interest & 0.5 & 1.9 & 2.7 & 2.6 & 2.4 & 1.3 & 0.2 & 0.0 \\
\hline \multicolumn{9}{|l|}{$\begin{array}{l}\text { Fund obligations based on existing and prospective credit } \\
\text { (in millions of SDRs) }\end{array}$} \\
\hline Principal & 17.1 & 15.2 & 14.1 & 14.4 & 103.4 & 198.4 & 178.8 & 84.0 \\
\hline Charges and interest & 0.5 & 3.9 & 9.7 & 13.9 & 13.7 & 9.4 & 3.3 & 0.9 \\
\hline \multicolumn{9}{|l|}{ Total obligations based on existing and prospective credit } \\
\hline In millions of SDRs & 17.6 & 19.1 & 23.8 & 28.3 & 117.1 & 207.8 & 182.1 & 84.9 \\
\hline In millions of US\$ & 26.4 & 29.6 & 35.8 & 42.4 & 175.8 & 312.5 & 274.2 & 127.7 \\
\hline In percent of gross international reserves & 1.9 & 1.8 & 2.3 & 2.9 & 12.3 & 18.9 & 14.4 & 6.0 \\
\hline In percent of exports of goods and services & 1.5 & 2.3 & 2.4 & 2.5 & 8.9 & 14.0 & 10.8 & 4.6 \\
\hline In percent of debt service $1 /$ & 48.4 & 40.1 & 37.8 & 39.9 & 71.9 & 80.8 & 77.8 & 46.8 \\
\hline In percent of GDP & 0.2 & 0.3 & 0.4 & 0.5 & 1.7 & 2.9 & 2.3 & 1.0 \\
\hline In percent of quota & 19.1 & 20.8 & 25.9 & 30.8 & 127.3 & 225.9 & 197.9 & 92.2 \\
\hline \multicolumn{9}{|l|}{ Outstanding Fund credit $1 /$} \\
\hline In millions of SDRs & 87.5 & 374.3 & 554.1 & 577.3 & 474.0 & 275.6 & 96.8 & 12.8 \\
\hline In billions of US\$ & 0.13 & 0.58 & 0.83 & 0.87 & 0.71 & 0.41 & 0.15 & 0.02 \\
\hline In percent of gross international reserves & 9.4 & 36.2 & 53.8 & 59.9 & 49.6 & 25.0 & 7.6 & 0.9 \\
\hline In percent of exports of goods and services & 7.6 & 45.6 & 56.0 & 50.5 & 36.1 & 18.6 & 5.8 & 0.7 \\
\hline In percent of debt service $1 /$ & 241.0 & 785.2 & 879.6 & 814.7 & 291.1 & 107.2 & 41.3 & 7.1 \\
\hline In percent of GDP & 1.1 & 6.2 & 9.3 & 9.2 & 7.1 & 3.8 & 1.2 & 0.1 \\
\hline In percent of quota & 95.1 & 406.8 & 602.2 & 627.5 & 515.2 & 299.5 & 105.2 & 13.9 \\
\hline Net use of Fund credit (millions of SDRs) & -12.5 & 286.8 & 179.8 & 23.3 & -103.4 & -198.4 & -178.8 & -84.0 \\
\hline Disbursements and purchases & 4.6 & 301.9 & 193.9 & 37.7 & 0.0 & 0.0 & 0.0 & 0.0 \\
\hline Repayments and repurchases & 17.1 & 15.2 & 14.1 & 14.4 & 103.4 & 198.4 & 178.8 & 84.0 \\
\hline \multicolumn{9}{|l|}{ Memorandum items: } \\
\hline Nominal GDP (in millions of US\$) & $11,916.6$ & $9,333.0$ & $8,980.4$ & $9,359.6$ & $10,049.3$ & $10,939.9$ & $12,026.1$ & $13,091.8$ \\
\hline Exports of goods and services (in millions of US\$) & $1,723.7$ & $1,272.9$ & $1,485.3$ & $1,713.8$ & $1,970.9$ & $2,231.0$ & $2,527.2$ & $2,797.1$ \\
\hline Gross international reserves (in millions of US\$) & $1,405.0$ & $1,604.5$ & $1,546.0$ & $1,445.7$ & $1,434.5$ & $1,654.6$ & $1,906.1$ & $2,115.4$ \\
\hline Debt service (in millions of US\$) $1 /$ & 54.6 & 73.9 & 94.5 & 106.2 & 244.4 & 386.7 & 352.6 & 273.0 \\
\hline Quota (millions of SDRs) & 92.0 & 92.0 & 92.0 & 92.0 & 92.0 & 92.0 & 92.0 & 92.0 \\
\hline
\end{tabular}

Sources: Fund staff estimates and projections.

1/ Total debt service includes IMF repurchases and repayments. 
Table 11. Armenia: Proposed Access, 2009

\begin{tabular}{|c|c|c|c|c|c|c|c|}
\hline & \multirow{3}{*}{$\begin{array}{l}\text { Proposed } \\
\text { Arrangement }\end{array}$} & \multicolumn{4}{|c|}{ Normal Access Cases } & \multicolumn{2}{|c|}{ High-Access Case } \\
\hline & & \multirow{2}{*}{$\begin{array}{l}\text { Proposed } \\
\text { Arrangement } \\
\text { (Percentile of } \\
\text { High Access } \\
\text { Cases) }\end{array}$} & \multirow[t]{2}{*}{$\begin{array}{c}\text { 20th } \\
\text { Percentile } \\
\end{array}$} & \multirow{2}{*}{$\begin{array}{c}\begin{array}{c}\text { 80th } \\
\text { Percentile }\end{array} \\
\text { (Ratio) }\end{array}$} & \multirow[t]{2}{*}{ Median } & \multirow[t]{2}{*}{$\begin{array}{c}\text { 20th } \\
\text { Percentile } \\
\end{array}$} & \multirow{2}{*}{$\begin{array}{c}\text { 80th } \\
\text { Percentile } \\
\text { (Ratio) }\end{array}$} \\
\hline & & & & & & & \\
\hline \multicolumn{8}{|l|}{ Access } \\
\hline In millions of SDRs & 533 & 14 & 34 & 406 & 128 & 1,273 & 12,862 \\
\hline Average annual access (arrangement amount in percent of quota) & 249 & 50 & 20 & 51 & 35 & 159 & 523 \\
\hline \multicolumn{8}{|l|}{ Total access in percent of: $2 /$} \\
\hline Actual quota & 580 & 57 & 30 & 75 & 49 & 300 & 921 \\
\hline Gross domestic product & 9 & 73 & 0.7 & 2.7 & 1.3 & 2.9 & 8.8 \\
\hline Gross international reserves & 51 & 58 & 5 & 43 & 13 & 27 & 83 \\
\hline Exports of goods and nonfactor services & 65 & 91 & 1.9 & 6.9 & 3.9 & 10.9 & 39.9 \\
\hline Imports of goods and nonfactor services & 23 & 60 & 1.7 & 6.4 & 3.6 & 10.2 & 35.1 \\
\hline \multicolumn{8}{|l|}{ Total debt stock } \\
\hline Of which: Public & 39 & $\ldots$ & $\ldots$ & $\ldots$ & $\ldots$ & 8 & 33 \\
\hline External & 45 & 98 & 2 & 6 & 3 & 7 & 19 \\
\hline Short-term 3/ & $\ldots$ & $\ldots$ & $\ldots$ & $\ldots$ & $\ldots$ & 20 & 87 \\
\hline M2 & 41 & 92 & 1 & 11 & 4 & 7 & 30 \\
\hline
\end{tabular}

Source: Executive Board documents, MONA database, and Fund staff estimates.

1/ High access cases include available data at approval and on augmentation for all the requests to the Board since 1995 which involved the use of the exceptional circumstances clause resources. Exceptional access augmentations are counted as separate observations. FCLs are also included. For the purpose of measuring access as a ratio of different metrics, access augmentations and previously approved and drawn amounts.

$2 /$ The data used to calculate ratios is the actual value for the year prior to approval for public and short-term debt, and the projection at the time of program approval for the year in which program was approved for all other variables. In Country Report No. 09/140 the data used to calculate all the relevant ratios is the actual value for the year (2008) prior to the approval of program.

3 / Refers to residual maturity. 


\section{AtTACHMENT I. ARMENiA: LetTER OF INTENT}

June 5, 2009

Mr. Dominique Strauss-Kahn

Managing Director

International Monetary Fund

Washington, D.C. 20431

Dear Mr. Strauss-Kahn:

After many years of strong macroeconomic performance, marked by rapid growth, significant poverty reduction, and low inflation, Armenia has been hit severely by the global economic crisis. The Government of the Republic of Armenia intends to build on the longlasting partnership with the IMF, which has led to many successes, to strengthen its program with sound macroeconomic policies and structural reforms that would alleviate the impact of the crisis and pave the way to a prompt recovery.

As the crisis was unfolding, a Stand-By Arrangement in the amount of SDR 368 million (400 percent of quota or \$544 million) for the period March 2009 through June 2011 was approved by the IMF Executive Board on March 6, 2009. The program supported by the Arrangement envisaged the return to a floating exchange rate on March 3, which has been successful in restoring confidence. The consequences of the dram depreciation on the financial system have been limited and the exchange rate has remained stable without large interventions by the Central Bank of Armenia.

However, the economic outlook has continued to deteriorate, and a larger contraction of economic activity is now expected for 2009. Balance of payments inflows have continued to decline, increasing external financing needs. On the fiscal side, tax revenues have fallen significantly, leading to a delay of budgeted government spending that compounds the drop in private sector demand.

The Government of Armenia requests the completion of the first review under the Stand-By Arrangement. Moreover, we request additional resources under the Stand-By Arrangement in the amount of SDR 165.6 million (180 percent of quota or about \$258 million) to cover the increased financing needs in 2009 and 2010. The objectives of the program remain unchanged: to help Armenia adjust to the changed outlook, restore confidence, and protect 
the poor. But these additional balance of payments resources are necessary to prevent excessive domestic adjustment, and thus preserve economic and financial stability and continued implementation of our budget while maintaining adequate social expenditure.

In addition, we request waivers for the non-observance of the end-March performance criteria on net banking system credit to the government and the program fiscal balance. These targets were missed due to the large unexpected decline in tax revenues, which we are redressing through a strengthening of our tax administration reform program. Furthermore, to adapt the conditionality to the current situation, we request the elimination of the indicative target on base money and the conversion of the end-June structural performance criterion (approval of a plan to increase the resources of the Deposit Guarantee Fund) to a structural benchmark. We also request the modification of end-June 2009, end-September 2009, and end-December 2009 quantitative performance criteria.

The attached Memorandum of Economic and Financial Policies outlines our policies for 2009 and 2010, which have been updated and revised to address the more challenging economic and financial situation. The Government believes that the policies set forth in the attached MEFP are adequate to achieve the objectives of the program, but it will take any other measure that may become necessary for this purpose. The Government will consult with the Fund on the adoption of additional measures, and in advance of revisions to the policies contained in the MEFP, in accordance with the Fund's policies on such consultation. Moreover, after the end of the arrangement, and as long as Armenia has outstanding financial obligations to the Fund, the Government will consult with the Fund on Armenia's economic and financial policies from time to time, at the initiative of the Government or at the request of the Managing Director. Finally, we grant our permission for the publication on the IMF's website of the staff report and this letter.

Very truly yours,

/s/

Tigran Sargsyan

Prime Minister

Republic of Armenia

/s/

Tigran Davtyan

Minister of Finance

Republic of Armenia /s/

Artur Javadyan

Chairman of the Central Bank

Republic of Armenia 


\section{Attachment II. Armenia: Memorandum of Economic and Financial Policies}

(June 5, 2009)

\section{RECENT DEVELOPMENTS AND OUTLOOK FOR 2009-10}

1. The economic outlook has deteriorated dramatically for Armenia. The global economic crisis has caused a swift turnaround in our economic prospects. After many years of rapid growth, Armenia is faced with a large contraction of economic activity on the back of declining external inflows and plummeting domestic demand. Falling remittances and rising unemployment are depressing household incomes and causing poverty to rise. In the first quarter of 2009, real GDP fell by 6.1 percent on an annual basis, and the contraction is expected to reach almost 10 percent for the year as a whole, with the construction sector and mining sector bearing the brunt of the crisis. Growth could resume, albeit moderately, in 2010 , provided that the expected global recovery materializes.

2. In this context, Armenia's external position is weakening. Exports are suffering both from the drop in external demand and from falling international commodity prices, and remittances are declining rapidly. This means that despite a fall in imports, the current account deficit is likely to worsen after reaching an estimated 11 percent of GDP in 2008, and the slowdown of capital inflows will add to financing pressures.

3. At the same time, government financing needs are increasing. Tax revenues fell by 15 percent in the first four months of 2009, compared with the same period in 2008 . Despite postponement of non-essential expenditures, we were not able to meet the endMarch fiscal targets under our IMF stand-by arrangement (SBA). Even with painful expenditure cuts, the fiscal deficit is expected to widen significantly for the year as a whole.

4. Encouragingly, pressures on the exchange rate have waned. On March 3, 2009, the Central Bank of Armenia (CBA) announced that it would return to a floating exchange rate and that it would stop intervening in the foreign exchange market except to smooth excessive volatility. At the same time, the CBA raised the policy rate by 100 basis points. As a result, the dram depreciated by 22 percent vis-à-vis the U.S. dollar and has been fairly stable since, despite limited intervention by the CBA. Bank deposits have remained stable, confirming the depositors' confidence in the banking system. In fact, banks are well capitalized, and have easily absorbed the balance sheet losses arising from the impact of the depreciation. International reserves have been strengthened by the provision of IMF resources. The conversion of dram deposits into foreign currency deposits, which had been intense prior to the depreciation, has stopped.

5. Despite the recent cuts in the policy rate, inflation is expected to remain low and credit conditions in the economy to remain tight. Year-on-year inflation increased to 
slightly over 3 percent in April from 1 percent in March-largely driven by increases in utility prices and some pass-through of the exchange rate depreciation-but is expected to remain low on the back of falling domestic demand. In the absence of significant pressures either on the exchange rate or inflation, the CBA started reducing its policy rate in April. Nonetheless, dram liquidity remains scarce, and banks have significantly cut back lending to the private sector, further dampening economic activity.

\section{The Program FOR 2009 ANd 2010}

6. The policies in our program have been updated and revised to reflect the more challenging outlook. The objectives of the program remain unchanged: namely, to help Armenia adjust to the changed external outlook, maintain confidence, and protect the poor. However, the sharp contraction in economic activity, widening fiscal deficit, and difficult conditions in credit markets require a robust policy response which will allow Armenia to return to high growth, a strong balance of payments, and continued progress in poverty reduction.

7. To this end, we intend to pursue the policies outlined below in the monetary and financial, fiscal, and structural areas. In particular, monetary and exchange rate policies are geared toward enabling the economy to adjust to the external shock and boosting confidence. Fiscal policies are likewise aimed at softening the impact of the economic downturn, ensuring medium-term fiscal sustainability, and protecting social services. And structural reforms are targeted at increasing the flexibility of the economy, and laying the basis for renewed growth and macroeconomic stability.

\section{A. Monetary, Exchange Rate, and Financial Sector Policies}

8. We are committed to maintaining a flexible exchange rate regime. The CBA will continue its current policy of intervening only to contain excessive volatility of the exchange rate, and will not resist movements that are in line with fundamentals. The CBA will avoid giving the market any perception of resuming targeting an exchange target level or range which could hinder the interplay of demand and supply. To introduce two-way risks in the foreign exchange market, we intend to rely on a coherent intervention strategy to achieve consistent and transparent outcomes. To this end, the CBA will adopt an intervention policy in accordance with the objectives of smoothing excessive movements of exchange rates and rebuilding foreign currency reserves (structural benchmark for end-June 2009). To enhance the transparency of its operations, the CBA commits to only conduct foreign exchange interventions with banks or government entities. Furthermore, to ensure proper exchange rate risk management, the $\mathrm{CBA}$ will consider tightening prudential measures regarding foreign currency exposures, both direct and indirect, including imposing limits on banks' open foreign exchange positions

9. With inflation pressures receding rapidly, the focus of monetary policy has now shifted. At the time of the depreciation, monetary policy was geared towards supporting the 
return to a flexible exchange rate regime. As the inflationary pressures triggered by the depreciation of the dram and the subsequent increases in utility prices are expected to subside in the face of extremely weak domestic demand, the CBA has been able to lower interest rates, providing some support to economic growth. Consistent with its primary objective of price stability and in line with its inflation forecasts, the CBA will continue to ease policy.

10. However, the economic crisis and the increased preference for foreign currency have reduced the effectiveness of monetary policy. Credit to the private sector has effectively dried up as banks tighten their credit standards and find fewer attractive lending opportunities. In addition, many banks, having experienced a shift toward foreign currency deposits, are trying to reduce the currency mismatch by extending foreign currency loans. On the other hand, borrowers, whose revenues are mostly in local currency, are reluctant to increase their own exchange rate exposure.

\section{Therefore, we intend to take steps to unlock the frozen credit market and} strengthen the transmission of monetary policy. In the short term, while maintaining sufficient short-term repo lending, we will endeavor to provide longer-term dram liquidity to facilitate banks' liquidity management by:

- $\quad$ (i) gradually introducing additional repo maturities of up to one year, including a 3month repo (structural benchmark for end June 2009);

- $\quad$ (ii) offering foreign exchange swaps as part of regular monetary instruments; and

- (iii) continuing to carry out outright purchase operations of government securities to the extent that these accommodate structural liquidity injections.

12. In the medium term, we will continue to develop the securities market as laid out in our capital market development program presented in the Memorandum of Economic and Financial Policies of March 2, 2009. We will increase the issuance of government securities in line with market demand and consistent with the memorandum of understanding between the CBA and the MoF, with a view to extending maturities. In addition, we will amend Article 11 of the "Law on the Central Bank of the Republic of Armenia" to ensure a gradual recapitalization of the CBA with marketable interest-bearing securities (structural benchmark for end September 2009). Finally, to support dedollarization of deposits, we are tightening the enforcement of the prohibition of payments in foreign currency.

13. We are also preparing to undertake exceptional credit easing operations. The CBA will use resources from the World Bank, KfW, and part of the resources from the Russian government to extend long-term domestic currency loans to banks for on-lending to small and medium enterprises (SMEs). We are also planning the creation of a secondary mortgage operator in partnership with the private sector. The new entity will raise financing 
through the issuance of domestic currency bonds and provide long-term dram loans to banks, collateralized on their mortgage portfolio cash flows.

14. We will continue to closely monitor the banking sector, particularly in light of the increase in nonperforming loans caused by the economic contraction and, to a lesser degree, by the depreciation. We will continue to conduct stress tests on each individual bank, focusing particularly on market, credit, and liquidity risk. The latest stress tests indicated that, even after the balance sheet losses owing to the depreciation, the banking system remains highly capitalized and liquid. In any case, we remain vigilant and ready to take steps to promptly address any potential vulnerabilities in the system.

\section{Existing legislation confers on the CBA comprehensive powers for intervening} and resolving banks. The CBA has strengthened its capacity to address banking crises by expanding the range of eligible collateral for emergency liquidity assistance. The CBA has also formalized a mechanism to support the recapitalization of banks through a subordinated debt facility matching bank shareholders' capital injections. The CBA also introduced a temporary loan facility to facilitate mergers and acquisitions. The CBA is securing public resources for this purpose. The CBA is considering requesting Fund technical assistance to further strengthen contingency planning and crisis preparedness.

\section{Enhancing the efficacy of the Deposit Guarantee Fund (DGF) will strengthen} our banking system safety net. The CBA and the DGF, with the support of technical assistance financed by KfW, will introduce a mechanism to periodically review the appropriate level of coverage of the deposit insurance for both dram and foreign currency deposits, including the possibility of equalizing their coverage. We are finalizing a plan to increase the resources of the DGF (structural benchmark for end June 2009). We are considering requesting technical assistance from the Fund on the possible adoption of riskadjusted deposit insurance premia.

\section{B. Fiscal Policy}

\section{We remain committed to fiscal discipline, which is necessary to maintain a} sustainable fiscal position in the medium term. Our program under the stand-by arrangement targeted a deficit of around 3 percent of GDP for 2009. As automatic stabilizers on the expenditure side are limited, greater discretionary expenditure is needed to avoid deepening the economic contraction. Therefore, we intend to keep spending in nominal terms in line with the stand-by arrangement, representing an increase as a percent of GDP relative to the program due to the fall in nominal GDP. On this basis, we expect a deficit of about 6.5 percent of GDP. Gradual adjustment will proceed in 2010, and to this end we will increase tax revenue by 0.4 percent of GDP and reduce expenditure by 0.6 percent of GDP, targeting a deficit of 5.8 percent of GDP for 2010 .

\section{Refocused and better-targeted public spending will be a key element of our} response to the crisis. On current spending, we intend to continue critical maintenance and 
operating expenditures, which will help strengthen public services and provide a positive economic impact. In addition, we are committed to preserving social spending and protecting the poor. Despite the pressures on overall expenditure, we commit to safeguard social spending by keeping the level of expenditure on social allowances and pensions at least as high as in the 2009 budget, which would imply an increase in social spending by $1 \frac{1}{2}$ percent of GDP. In addition:

- In close collaboration with the World Bank, we will develop a strategy to further strengthen the targeting of social safety nets (structural benchmark for end December 2009), and expect to achieve sizable savings in these areas, which will be directed to providing assistance to the newly-unemployed and strengthening the family benefit program.

- We will increase spending on infrastructure projects such as road rehabilitation-a critical element of our countercyclical response-which will generate employment, notably for the poor.

19. We expect external sources to fund additional capital spending and credit lines for SMEs. We anticipate the disbursement of a \$500 million loan from Russia by mid-2009, which will be spent on critical projects, consistent with our capacity to implement these projects. The loan will primarily finance new housing projects in the earthquake-affected zone (Gyumri,Vanadzor, and Spitak), thus resettling the remaining 6,000 families left without permanent housing since the catastrophic 1988 earthquake. The loan will also finance SME lending via the banking system, helping offset the credit freeze, and fund identified infrastructure projects. These measures will provide much-needed stimulus to our economy, help accelerate the path to recovery, and address critical social needs.

20. We intend to increase domestic financing moderately, to address financing needs in addition to supporting financial intermediation. We plan to increase financing from issuing government securities in both 2009 and 2010, subject to market demand. Given the limited appetite currently for longer-maturity securities, we expect that some of these may be purchased by the CBA in the secondary market. Moderate net issuance of government securities is not expected to crowd out the private sector. We will limit the use of government cash deposits to finance the budget, as we recognize the need to have a minimum buffer for cash flow management of at least AMD 30 billion by end 2009 .

\section{Fiscal structural reforms}

\section{Our tax administration reform program is a critical element of our response to} the crisis and underpins our effort to strengthen fiscal sustainability. In the first quarter, we reduced the stock of tax credits from AMD 154.4 billion to AMD 147 billion, and will further reduce it in the course of 2009, which will also provide stimulus during the downturn. More generally, we are committed to analyzing and fixing the systemic problems underlying 
these tax credits in the context of our comprehensive tax administration reform. We also remain committed to introducing best practices in VAT refund processing to exporters by:

- $\quad$ (i) meeting the statutory 90 day processing deadline for all VAT refund claims filed in 2009 (to be monitored on a continuous basis);

- $\quad$ (ii) Reducing to zero the stock of VAT refund claims that were not processed within the statutory 90 -day processing deadline;

- (iii) implementing risk-based auditing for VAT refunds; and

- (iv) submitting legislation to parliament to (a) strengthen penalties for overstated VAT refund claims effective January 2010, and (b) pay interest on legitimate late refunds arising from claims filed after December 2009 with interest to be paid out of VAT revenues (structural benchmark for end September 2009).

22. We have also accelerated tax administration reforms in additional areas. In particular: (i) tax return filing commissions have been liquidated and mailboxes installed in tax inspectorates for receiving tax returns; (ii) the new structure and functions of the regional inspectorates have been created; (iii) based on actual performance indicators, we are developing a system for the assessment of tax inspectorates and structural units' performance, and will continue the consolidation of tax inspectorates after assessing the results of the first round of consolidation; (iv) we are preparing the requirements to perform risk-based audits; and (v) we are introducing electronic filing of taxes.

\section{We have introduced some tax policy changes aimed at increasing revenue,} including raising the presumptive tax on tobacco. In addition, we remain committed to abolishing the presumptive taxation for tobacco and fuel. To this end, we will submit legislation to parliament to bring all tobacco and petroleum products within the regular tax regimes (excises, custom duties, profit tax and VAT), effective January 2011 (structural benchmark for end December 2009).

\section{In addition:}

- We are continuing to progress on the introduction of the funded pension pillar. We will revise our estimates of the costs of this reform, as well as those associated with the planned increase in basic pensions, taking into account the deteriorating economic outlook. Legislation on the new pension system, including on the unification of income tax and social contributions, will be submitted to parliament shortly.

- We intend to produce an analytical report on medium term fiscal risks, including those arising from the introduction of the funded pillar and the costs associated with the planned increase in basic and average pensions over the next few years. To further improve our fiscal framework, we also intend to strengthen debt management. 
- The special part of the unified tax code - which covers tax policy - has been drafted and is expected to be submitted to Parliament by September 2009.

\section{Other structural reforms}

25. The government is also taking numerous structural measures to strengthen the business climate as part of its anti-crisis action plan. In particular, we are in the process of creating one-stop shops in the corporate and service sectors, establishing free economic zones at Zvartnots international airport and in the city of Gyumri, and strengthening the Committee on the Protection of Economic Competition to improve domestic competition and reduce import monopolies. The business climate will be further strengthened by the tax administration reforms mentioned above and by our efforts to fight against corruption. Indeed, we are introducing legislative changes on prescribing targeted severe sanctions for senior state officials engaging in business activities. World Bank support in various areas (including regulatory changes, infrastructure development, competition policy, and customs reform) should contribute to enhancing competitiveness.

\section{Program FinanCing}

26. Armenia faces larger than anticipated balance of payments financing needs over the next two years. The steep contraction in export earnings and remittances, along with the projected reduction in FDI inflows has severely weakened the economy. Despite a larger than anticipated contraction in imports, projected financing gaps over the program period have increased relative to the original framework of the program by about $\$ 350$ million for 20092010.

27. The amount of resources available from donors is insufficient to cover these gaps. The World Bank plans to provide \$545 million in 2009-2012 with high upfront IDA budget support disbursements in 2009. The Asian Development Bank is likely to provide about $\$ 130$ million in 2009-10, of which at least $\$ 70$ million will be provided for direct budget support. A portion of these resources are new relative to the original program in 2009, and thus will help to address the increased balance of payments financing needs. In addition, we now anticipate budget support from the European Union in 2010. Finally, the expected Russian loan for project support will be used to provide on-lending to SMEs, infrastructure investment, and housing construction, although it is not intended for budget support. Given the magnitude of the external shock and the marked effect on Armenia's external and domestic economy, expected resources are insufficient to cover the widening financing gap.

28. The financing needs are particularly acute in 2009 and 2010. Absent additional Fund financing, Armenia has limited options to finance the remaining gap and would experience protracted weakness in the balance of payments, as domestic demand would have to contract by an unsustainably high amount. We anticipate limited financing needs in 2011. 


\section{Program Monitoring}

29. Given the significant external financing needs, we would like to request an augmentation of the current Stand By Arrangement in the amount of 180 percent of quota (SDR 165.6 million or about $\$ 258$ million), which would increase Fund financing to SDR 533.6 million, about $\$ 830$ million. This would result in increased access under the 28month arrangement to 580 percent of quota. The requested funding would cover the remaining financing gap for 2009 and a large portion of the remaining balance of payments deficit in 2010 and 2011. The increased financing will be used to support international reserves as Armenia adjusts to the worsened economic outlook.

30. The program will continue to be monitored via quarterly performance criteria, indicative targets, and structural benchmarks. The quantitative criteria and indicative targets are outlined in the attached Table 1, and the structural benchmarks are listed in Table 2. The structural measures discussed above that are not mentioned in Table 2 are part of the authorities' overall reform effort and will be considered in the context of program reviews, but is not part of conditionality under this program. The second review of the program is expected to be completed on or after August 15, 2009, the third review on or after November 15, 2009, and the fourth review on or after February 15, 2010. 
Table 1. Armenia: Quantitative Targets, 20091

(in billions of drams, unless otherwise specified)

\begin{tabular}{|c|c|c|c|c|c|c|c|c|c|c|}
\hline & \multirow{3}{*}{$\begin{array}{l}2008 \\
\text { Dec. }\end{array}$} & \multicolumn{9}{|c|}{2009} \\
\hline & & \multicolumn{3}{|c|}{ Mar. } & \multicolumn{2}{|c|}{ Jun. } & \multicolumn{2}{|l|}{ Sep. } & \multicolumn{2}{|l|}{ Dec. } \\
\hline & & $\begin{array}{c}\text { Country Report } \\
\text { No. } 09 / 140\end{array}$ & Adj. Prog. & Prel. & $\begin{array}{c}\text { Country Report } \\
\text { No. 09/140 }\end{array}$ & Prog. & $\begin{array}{c}\text { Country Report } \\
\text { No. } 09 / 140\end{array}$ & Prog. & $\begin{array}{c}\text { Country Report } \\
\text { No. 09/140 }\end{array}$ & Prog. \\
\hline \multicolumn{11}{|l|}{ Performance Criteria } \\
\hline Net official international reserves (floor, in millions of dollars) & $1,084.7$ & 455.9 & & 588.5 & 455.9 & $1,008.3$ & 505.9 & 923.3 & 568.4 & 839.8 \\
\hline Net domestic assets of the CBA (ceiling) $2 /$ & 75.9 & 148.9 & 143.6 & 55.6 & 191.3 & -117.8 & 195.5 & -60.0 & 196.1 & 24.5 \\
\hline Net banking system credit to the government (ceiling) & -37.3 & -23.6 & & -10.6 & -2.7 & -217.6 & -1.8 & -163.7 & 9.4 & -118.6 \\
\hline Program fiscal balance (floor) $/ 3$ & -73.1 & -31.9 & -29.9 & -36.6 & -73.3 & -123.4 & -102.2 & -212.3 & -142.8 & -287.4 \\
\hline External arrears (continuous criterion) & 0 & 0 & & 0 & 0 & 0 & 0 & 0 & 0 & 0 \\
\hline \multicolumn{11}{|l|}{ Indicative Targets } \\
\hline Reserve money (ceiling) $4 /$ & 449.7 & 381.3 & & 391.7 & 419.8 & $\ldots$ & 439.5 & $\ldots$ & 464.1 & $\ldots$ \\
\hline Stock of tax credits (ceiling) & 154.4 & 154.4 & & 147.0 & 154.4 & 147.0 & 145.0 & 145.0 & 145.0 & 145.0 \\
\hline
\end{tabular}

1/ All items as defined in the TMU. The figures in bold indicate when a target has not been met.

2/ At program exchange rates.

4/ Indicative target up to end-March 2009 . 
Table 2. Armenia: Proposed Structural Benchmarks for the Second, Third, and Fourth Reviews

\begin{tabular}{|c|c|c|}
\hline Item & Measure & $\begin{array}{l}\text { Proposed Time } \\
\quad \text { Frame } \\
\text { (End of Period) }\end{array}$ \\
\hline & $\underline{\text { Financial sector }}$ & \\
\hline 1 & Approve a plan to increase the resources of the Deposit Guarantee Fund. & June 2009 \\
\hline 2 & $\begin{array}{l}\text { CBA to adopt a formal foreign exchange market intervention strategy consistent with IMF technical } \\
\text { assistance. }\end{array}$ & June 2009 \\
\hline 3 & Start offering repo operations with a maturity of at least three months. & June 2009 \\
\hline \multirow[t]{2}{*}{4} & $\begin{array}{l}\text { Submit to parliament an amendment to Article } 11 \text { of the "Law on the Central Bank of the Republic of Armenia" } \\
\text { to ensure a gradual recapitalization of the CBA with marketable interest bearing securities. }\end{array}$ & September 2009 \\
\hline & $\underline{\text { Tax administration }}$ & \\
\hline 5 & Meet the statutory 90-day processing deadline for all VAT refund claims filed in 2009. & Continuous in 2009 \\
\hline \multirow[t]{2}{*}{6} & $\begin{array}{l}\text { Submit legislation to parliament to (a) strengthen penalties for overstated VAT refund claims effective January } \\
2010 \text {, and (b) pay interest on all legitimate VAT refund claims filed after December } 2009 \text { and not refunded } \\
\text { within } 90 \text { days, with interest to be paid out of VAT revenues. }\end{array}$ & September 2009 \\
\hline & $\underline{\text { Tax and social policy }}$ & \\
\hline 7 & $\begin{array}{l}\text { In close collaboration with the World Bank, develop a strategy to further strengthen the targeting of social } \\
\text { safety nets. }\end{array}$ & December 2009 \\
\hline 8 & $\begin{array}{l}\text { As part of the unified Tax Code, submit legislation to parliament to bring petroleum and tobacco products } \\
\text { within the regular tax regimes (excise tax, customs duties, profit tax, and VAT), effective January } 2011 .\end{array}$ & December 2009 \\
\hline
\end{tabular}




\section{AtTACHMENT III. ARMEnia: TeChNiCAL Memorandum OF Understanding}

This memorandum defines the benchmarks, performance criteria, indicative targets, adjusters, and reporting modalities referred to in the Memorandum of Economic and Financial Policies (MEFP).

\section{QUANTITATIVE TARGETS}

1. The program targets a minimum level of net official international reserves (NIR) of the Central Bank of Armenia (CBA; performance criterion). The stock of such reserves will be calculated as the difference between total official gross international reserves (excluding reserve money denominated in foreign currencies) and official gross reserve liabilities. Total gross official international reserves are defined as the CBA's holdings of monetary gold (excluding amounts pledged as collateral or in swaps), holdings of Special Drawing Rights (SDRs), any reserve position in the IMF, and holdings of convertible currencies in cash or in nonresident financial institutions (deposits, securities, or other financial instruments). Gross reserves held in the form of securities and other financial instruments are marked to market. Gross reserves are reported separate from the balance on the government's Special Privatization Account (SPA) and the Millennium Challenge Account (MCA) and exclude capital subscriptions in foreign financial institutions and illiquid foreign assets. Official reserve liabilities shall be defined as the total outstanding liabilities of the government and the CBA to the IMF and convertible currency liabilities of the CBA to nonresidents with an original maturity of up to and including one year. NIR is monitored in U.S. dollars, and, for program monitoring purposes, assets and liabilities in currencies other than the U.S. dollar shall be converted into dollar-equivalent values using the exchange rates as specified in Table 1.

2. The program targets a maximum level of net domestic assets (NDA) of the CBA (performance criterion). For program purposes, NDA is defined as reserve money minus NIR, minus reserve money denominated in foreign currencies, plus medium- and long-term liabilities (i.e. liabilities with a maturity of one year or more) of the CBA, plus the balance of outstanding Fund purchases credited directly to the government account at the CBA. To evaluate program targets, the dram-equivalent values of NIR, medium- and long-term liabilities, and reserve money in U.S. dollar are calculated at the program exchange rate of dram 385 per U.S. dollar. The dram-equivalent value of reserve money in euro is calculated at the program exchange rate specified in Table 1. NDA is composed of net CBA credit to the general government; outstanding credit to domestic banks by the CBA (including overdrafts) minus liabilities not included in reserve money (exclusive of accrued interest), 
and other items net. Reserve money is defined as the sum of currency issued, required and excess reserves, and current and time deposit accounts of certain resident agents. ${ }^{5}$

3. The program targets a maximum level of net banking system credit to the government (performance criterion), defined as the sum of net credit from the CBA and net credit from commercial banks to the central government.

- The stock of net credit from the CBA to the government, which includes the CBA's holdings of treasury bills and treasury bonds less all types of government deposits with the CBA (including the deposits in the Treasury Single Account, deposits of donor-financed project implementation units, the Lincy foundation, and balances of proceeds from the sale of humanitarian assistance). Treasury bonds are valued at the purchase price and excluding accrued interest, and treasury bills are valued at the purchase price plus the implicit accrued interest.

- Net credit from commercial banks to the government includes: (1) gross commercial bank credit to the central government less government deposits with commercial banks (including the counterpart funds of certain government on lending to the economy financed by the Lincy Foundation and the World Bank); and (2) bank holdings of treasury bonds (valued at the purchase price and excluding accrued interest) and treasury bills (valued at the purchase price plus the implicit accrued interest).

4. The program imposes a zero ceiling on external payment arrears (continuous performance criterion) defined as all unpaid debt-service obligations (i.e., payments of principal and interest) arising in respect of public sector loans contracted or guaranteed including unpaid penalties or interest charges associated with these obligations that are overdue beyond 30 days after the due date. ${ }^{6}$

\section{The program targets a minimum level of the program fiscal balance} (performance criterion). The fiscal balance is measured as the negative of the sum of net domestic banking system credit to the government, domestic nonbank net financing, and external net financing to the government. Should a general subsidy be introduced off-budget, the overall balance will be measured including the subsidy as part of government spending.

Net banking system credit to the government equals the change during the period of net credit to the government.

\footnotetext{
${ }^{5}$ Liquidity absorbing transactions under reverse repurchase agreements, the CBA's deposit facility, foreign currency swaps, and securities issued by the CBA are excluded from the reserve money definition.

${ }^{6}$ The public sector is defined following the Government Financial Statistics Manual (GFS 2001) and System of National Accounts (1993 SNA). It includes the general government and nonfinancial public enterprises.
} 
- Nonbank net financing equals the sum of: (1) the change during the period of outstanding treasury bills and bonds to nonbanks (including accrued interest for treasury bills and excluding accrued interest for treasury bonds); $;^{7}$ (2) any other disbursement or transaction that increases nonbanks' claims on the central government plus withdrawals from the special privatization account or the treasury sub-account containing privatization proceeds in dram, less amortizations made by the central government to private resident nonbank agents.

- External net financing equals total debt-increasing disbursements from nonresidents to the central government (including Fund net purchases credited directly to the government accounts at the CBA) less total amortizations from the central government to non-residents. All foreign-currency denominated transactions are recorded in drams using the prevailing exchange rate at the time of the transaction.

6. The project implementation units, which carry out projects financed by the US-based Lincy Foundation, maintain accounts at the CBA. These grants are recorded in the fiscal accounts as external grants on the revenue side and as foreign-financed capital expenditure on the expenditure side. In addition, any loans extended by the U.S.-based Lincy foundation to finance investments and that are intermediated through the banking system are recorded in the financial accounts as a financing item below the line and are thus excluded from net lending.

7. Foreign currency proceeds from selling enterprises are deposited into the Special Privatization Account (SPA). The account is held at the CBA and the proceeds are invested abroad together with the CBA's international reserves. These proceeds are included in the definition of the monetary accounts of the CBA as part of net foreign assets with a counter entry in other items net. Any budgeted withdrawal from the SPA will be accounted for as privatization proceeds used to finance the budget and will be recorded below the line. Any unanticipated withdrawal from the SPA will be recorded below the line as privatization receipts; these withdrawals, however, will be replenished during the same fiscal year. Domestic currency proceeds from selling enterprises to residents are deposited in a subaccount of the treasury single account.

\section{The program targets a maximum level of stock of tax credits (indicative target)} defined as the sum of outstanding accumulated credit by the State Revenue Committee (SRC) of all types of tax revenues (VAT, profit tax, excises, income tax, presumptive payments, and others) resulting from advanced tax payments to be offset against future tax liabilities.

\footnotetext{
${ }^{7}$ Domestic nonbank holdings of treasury bills and treasury bonds are defined as total outstanding treasury bills and bonds less holdings by the banking system and the SFSI.
} 


\section{AdJUSTERS}

9. The quantitative performance criteria and indicative targets under the program are subject to the following adjusters, calculated, where relevant, using program exchange rates:

- Changes in reserve requirements: The ceiling on the NDA of the CBA will be adjusted downward (upward) by the amount of banks' reserves freed (seized) by any reduction (increase) of the reserve requirement ratio on both domestic currency and foreign currency deposits relative to the baseline assumption as per the following formula: $\Delta \mathrm{NDA}=\Delta \mathrm{rB}$, where $\mathrm{B}$ denotes the level of the reservable deposits in the initial definition and $\Delta \mathrm{r}$ is the change in the reserve requirement ratio.

- KfW and World Bank loan disbursements: the ceiling on the NDA of the CBA will be adjusted upward (downward) by the full amount of any excess (shortfall) of disbursements from the KfW and World Bank loans directed at SME financing compared to programmed amounts (Table 2). The floor on NIR will be adjusted upward (downward) by the cumulative amount of any excess (shortfall) of these disbursements compared to program amounts.

- External financing to the public sector, defined as disbursements of loans from bilateral and multilateral agencies for budget, project support, and the \$500 million Russian loan (including Fund purchases credited directly to the government accounts at the CBA), with the exception of the KfW and World Bank disbursements mentioned above:

o The floor on NIR will be adjusted upward (downward) by the cumulative amount of any excess (shortfall) of external financing in the form of budget support (excluding Fund disbursements to the government) or the Russian project loan compared to program amounts (Table 3).

- The ceiling on NDA will be adjusted upward by the amount of any shortfall of external financing in the form of budget support or from the Russian loan compared to program amounts.

- The ceiling on net banking sector credit to government will be adjusted upward by the cumulative amount of any shortfall of total external financing compared to programmed amounts (Table 3 ).

- The floor on the program fiscal balance on a cash basis will be adjusted downward by the cumulative amount of any excess of total external financing compared to programmed amounts (Table 3). 
- Recapitalization of the CBA: the ceiling on net credit of the banking system to the government will be adjusted upward by the full amount of the recapitalization of the CBA. 


\section{DATA REPORTING}

The government will provide the IMF the information specified in the following table.

\begin{tabular}{|c|c|c|c|c|}
\hline $\begin{array}{c}\text { Reporting } \\
\text { Agency }\end{array}$ & Type of Data & Description of Data & Frequency & Timing \\
\hline \multirow[t]{11}{*}{$\mathrm{CBA}^{8}$} & CBA balance sheet & Summary & Daily & $\begin{array}{l}\text { The following } \\
\text { working day }\end{array}$ \\
\hline & CBA balance sheet & $\begin{array}{l}\text { Summary at program exchange rates; } \\
\text { and by chart of accounts at actual } \\
\text { official exchange rates }\end{array}$ & Monthly & $\begin{array}{l}\text { Within seven days } \\
\text { of the end of each } \\
\text { month }\end{array}$ \\
\hline & Monetary survey & $\begin{array}{l}\text { Summary banking system balance sheet } \\
\text { for the central bank at both program } \\
\text { exchange rates and by chart of accounts } \\
\text { at actual official exchange rates; the } \\
\text { consolidated balance sheet of } \\
\text { commercial banks by chart of accounts } \\
\text { at actual official exchange rates }\end{array}$ & Monthly & $\begin{array}{l}\text { Within } 25 \text { days of } \\
\text { the end of each } \\
\text { month }\end{array}$ \\
\hline & International reserves & $\begin{array}{l}\text { By chart of accounts; at (i) program } \\
\text { exchange rates; and (ii) at actual } \\
\text { official exchange rates }\end{array}$ & Daily & $\begin{array}{l}\text { The following } \\
\text { working day }\end{array}$ \\
\hline & $\begin{array}{l}\text { Foreign exchange } \\
\text { market }\end{array}$ & $\begin{array}{l}\text { Official exchange rates (buying and } \\
\text { selling); daily trade volume, and } \\
\text { weighted average exchange rate of the } \\
\text { interbank, intrabank and Nasdaq-OMX }\end{array}$ & Daily & Within 1 day \\
\hline & $\begin{array}{l}\text { Foreign exchange } \\
\text { market }\end{array}$ & $\begin{array}{l}\text { CBA foreign exchange operations, } \\
\text { (exchange rate, volume, counterpart) }\end{array}$ & Daily & Within 1 day \\
\hline & $\begin{array}{l}\text { Foreign exchange } \\
\text { market }\end{array}$ & Exchange rate (buying and selling) & Daily & Within 1 day \\
\hline & Interest rates & Refinance rate & $\begin{array}{l}\text { At least } \\
\text { monthly }\end{array}$ & $\begin{array}{l}\text { Within } 1 \text { days of } \\
\text { the CBA Board } \\
\text { decision }\end{array}$ \\
\hline & $\begin{array}{l}\text { Interbank money } \\
\text { market }\end{array}$ & $\begin{array}{l}\text { Daily interbank repo volume and } \\
\text { interest rate and number of trades }\end{array}$ & Daily & Within 1 day \\
\hline & CBA operations & $\begin{array}{l}\text { Repo (reverse repo) operations, open } \\
\text { market operations, Lombard credits, } \\
\text { deposit facility, and foreign exchange } \\
\text { swaps (volumes, maturity, yields, } \\
\text { exchange rates) }\end{array}$ & Daily & Within 1 day \\
\hline & Bank liquidity & $\begin{array}{l}\text { Reserves and excess reserves, by } \\
\text { currency }\end{array}$ & Monthly & $\begin{array}{l}\text { Within } 15 \text { days of } \\
\text { the end of each } \\
\text { reference period }\end{array}$ \\
\hline
\end{tabular}

\footnotetext{
${ }^{8}$ As defined in CBA resolution No. 201 (December 6, 1999).
} 


\begin{tabular}{|c|c|c|c|c|}
\hline & $\begin{array}{l}\text { Interest rates and flows } \\
\text { of the funds attracted } \\
\text { and allocated by } \\
\text { commercial banks }\end{array}$ & By currency and maturity & Weekly & $\begin{array}{l}\text { Last working day } \\
\text { of the week }\end{array}$ \\
\hline & $\begin{array}{l}\text { T-bill and coupon bond } \\
\text { financing, CBA } \\
\text { securities }\end{array}$ & $\begin{array}{l}\text { Auction data: date, original and } \\
\text { remaining maturities, issuance volume, } \\
\text { allocation, average yield and coupon } \\
\text { yield (if available) }\end{array}$ & Weekly & $\begin{array}{l}\text { Last working day } \\
\text { of the week }\end{array}$ \\
\hline & Banking data & $\begin{array}{l}\text { Sectoral distribution of loans and } \\
\text { deposits; dollarization of loans and } \\
\text { deposits; loan maturities; interbank } \\
\text { rate, by volume and maturity; T-bill } \\
\text { rate, bond yield; deposit and lending } \\
\text { rates, by maturity; monthly weighted } \\
\text { average interest rate on government } \\
\text { bonds }\end{array}$ & Monthly & $\begin{array}{l}\text { Within } 25 \text { days of } \\
\text { the end of each } \\
\text { month }\end{array}$ \\
\hline & Banking indicators & $\begin{array}{l}\text { Capital adequacy; asset composition } \\
\text { and quality; profitability; liquidity; } \\
\text { open FX positions; and compliance } \\
\text { with prudential norms }\end{array}$ & Monthly & $\begin{array}{l}\text { Within } 30 \text { days of } \\
\text { the end of each } \\
\text { month }\end{array}$ \\
\hline & $\begin{array}{l}\text { Banking sector stress } \\
\text { tests }\end{array}$ & $\begin{array}{l}\text { Results of stress tests on exchange rate, } \\
\text { liquidity, and credit risk }\end{array}$ & Monthly & $\begin{array}{l}\text { Within } 30 \text { days of } \\
\text { the end of each } \\
\text { month }\end{array}$ \\
\hline & CPI & Index of core inflation & Monthly & $\begin{array}{l}\text { Within } 21 \text { days of } \\
\text { the end of each } \\
\text { month }\end{array}$ \\
\hline & Transfers & $\begin{array}{l}\text { Non-commercial transfers of } \\
\text { individuals }\end{array}$ & Monthly & $\begin{array}{l}\text { Within } 30 \text { of the } \\
\text { end of each month }\end{array}$ \\
\hline & Other monetary data & IFS format & Monthly & $\begin{array}{l}\text { Within } 45 \text { days of } \\
\text { the end of each } \\
\text { month }\end{array}$ \\
\hline $\begin{array}{l}\text { Ministry of } \\
\text { Finance }\end{array}$ & $\begin{array}{l}\text { T-bill and coupon bond } \\
\text { financing }\end{array}$ & $\begin{array}{l}\text { By holders, i.e., CBA, resident banks, } \\
\text { resident nonbanks, and nonresidents }\end{array}$ & Monthly & $\begin{array}{l}\text { Within } 7 \text { days of } \\
\text { each month }\end{array}$ \\
\hline (MOF) & External debt & $\begin{array}{l}\text { Disbursements and stock of outstanding } \\
\text { short-term and contracting or } \\
\text { guaranteeing and outstanding stock of } \\
\text { medium-and long-term external debt of } \\
\text { the government, the CBA, and state- } \\
\text { owned companies (by company); any } \\
\text { stock of arrears on external debt service } \\
\text { and outstanding stock of government } \\
\text { guarantees and external arrears }\end{array}$ & Monthly & $\begin{array}{l}\text { Within } 21 \text { days of } \\
\text { the end of each } \\
\text { month (preliminary } \\
\text { data ) and within } \\
45 \text { days of the end } \\
\text { of each month } \\
\text { (final data) }\end{array}$ \\
\hline & Revenue collection & $\begin{array}{l}\text { Total revenue collected separately by } \\
\text { the tax administration and customs } \\
\text { administration, including revenue by } \\
\text { individual tax, and social contributions }\end{array}$ & Monthly & $\begin{array}{l}\text { Within } 7 \text { days of } \\
\text { the end of each } \\
\text { month }\end{array}$ \\
\hline & Expenditure arrears & Government & Monthly & $\begin{array}{l}\text { Within } 45 \text { days of } \\
\text { the end of each } \\
\text { month for } \\
\text { government arrears }\end{array}$ \\
\hline
\end{tabular}




\begin{tabular}{|c|c|c|c|c|}
\hline & Privatization receipts & $\begin{array}{l}\text { Balance on the SPA; gross inflows into } \\
\text { and outflows from the SPA during the } \\
\text { month, specifying the nature of each } \\
\text { transaction }\end{array}$ & Monthly & $\begin{array}{l}\text { Within } 7 \text { days of } \\
\text { the end of each } \\
\text { month }\end{array}$ \\
\hline & $\begin{array}{l}\text { Treasury single account } \\
\text { (TSA) }\end{array}$ & $\begin{array}{l}\text { Detailed breakdown of central treasury } \\
\text { account, including deposits at the } \\
\text { central treasury, community budgets, } \\
\text { off budget account, monetization } \\
\text { account, state budget account and the } \\
\text { Republic correspondent account-- } \\
\text { flows during the month and end of } \\
\text { month stocks. }\end{array}$ & Monthly & $\begin{array}{l}\text { Within } 7 \text { days of } \\
\text { the end of each } \\
\text { month }\end{array}$ \\
\hline & $\begin{array}{l}\text { Consolidated central } \\
\text { government }\end{array}$ & State budget & Monthly & $\begin{array}{l}\text { Within } 30 \text { days of } \\
\text { the end of each } \\
\text { month }\end{array}$ \\
\hline & $\begin{array}{l}\text { Consolidated general } \\
\text { government }\end{array}$ & $\begin{array}{l}\text { Central and local governments, and } \\
\text { Non-Commercial Enterprises that } \\
\text { belong within the general government } \\
\text { (NCEs) }\end{array}$ & Quarterly & $\begin{array}{l}\text { Within } 60 \text { days of } \\
\text { the end of each } \\
\text { quarter }\end{array}$ \\
\hline & $\begin{array}{l}\text { Consolidated general } \\
\text { government }\end{array}$ & $\begin{array}{l}\text { Central and local governments, and } \\
\text { NCEs that belong within the general } \\
\text { government }\end{array}$ & Annual & $\begin{array}{l}\text { Within } 180 \text { days of } \\
\text { the end of each } \\
\text { year }\end{array}$ \\
\hline & Budget execution & $\begin{array}{l}\text { All cash receipts, cash expenditures, } \\
\text { including domestic and external debt- } \\
\text { service payments, external and } \\
\text { domestic borrowing operations, and } \\
\text { inflow of grants to the central } \\
\text { government; expenditure data will be } \\
\text { provided according to both economic } \\
\text { and functional classifications, } \\
\text { consistent with the GFSM2001 } \\
\text { methodology }\end{array}$ & Monthly & $\begin{array}{l}\text { Within one month } \\
\text { following the end } \\
\text { of each quarter. }\end{array}$ \\
\hline \multirow[t]{4}{*}{ NSS } & \multirow[t]{2}{*}{ Balance of payments } & Detailed export and import data & Monthly & $\begin{array}{l}\text { Within } 28 \text { days of } \\
\text { the end of each } \\
\text { month }\end{array}$ \\
\hline & & Detailed export and import data & Quarterly & $\begin{array}{l}\text { Within } 45 \text { days of } \\
\text { the end of each } \\
\text { quarter }\end{array}$ \\
\hline & GDP & Estimates & Monthly & $\begin{array}{l}\text { Within } 30 \text { days of } \\
\text { the end of each } \\
\text { month }\end{array}$ \\
\hline & CPI & By category & Monthly & $\begin{array}{l}\text { Within } 5 \text { days of } \\
\text { the end of each } \\
\text { month }\end{array}$ \\
\hline $\begin{array}{l}\text { State } \\
\text { Revenue } \\
\text { Committee }\end{array}$ & Tax arrears & By type of tax & Monthly & $\begin{array}{l}\text { Within } 30 \text { days of } \\
\text { the end of each } \\
\text { quarter (monthly } \\
\text { data provided on a } \\
\text { quarterly basis) }\end{array}$ \\
\hline
\end{tabular}




\begin{tabular}{|c|c|c|c|}
\hline & $\begin{array}{l}\text { For or the } 30 \text { largest debtors and for all } \\
\text { major companies in the energy, water, } \\
\text { and irrigation sectors }\end{array}$ & Quarterly & $\begin{array}{l}\text { Within } 30 \text { days of } \\
\text { the end of each } \\
\text { quarter }\end{array}$ \\
\hline Tax credits & $\begin{array}{l}\text { Detailed data, by type of tax, of } \\
\text { outstanding tax credits for all types of } \\
\text { tax revenues }\end{array}$ & Monthly & $\begin{array}{l}\text { Within } 45 \text { days of } \\
\text { the end of each } \\
\text { month (monthly } \\
\text { data provided on a } \\
\text { quarterly basis) }\end{array}$ \\
\hline $\begin{array}{l}\text { VAT refund claims in } \\
\text { arrears }\end{array}$ & $\begin{array}{l}\text { Detailed data on VAT refunds in } \\
\text { arrears which include all outstanding } \\
\text { VAT refunds that have not been } \\
\text { accepted (and refunded), or offset (in } \\
\text { full or in part), or rejected (in full or in } \\
\text { part) after the } 90 \text { day statutory } \\
\text { processing period. Number of refund } \\
\text { applications processed per month. }\end{array}$ & Monthly & $\begin{array}{l}\text { Within } 45 \text { days of } \\
\text { the end of each } \\
\text { month (monthly } \\
\text { data provided on a } \\
\text { quarterly basis) }\end{array}$ \\
\hline Import data & $\begin{array}{l}\text { 1. Total value of recorded imports, } \\
\text { breaking out raw diamond imports; } \\
\text { 2. Total value of non-duty free } \\
\text { recorded imports; } \\
\text { 3. Number of total transactions } \\
\text { involving recorded imports; } \\
\text { 4. Number of total transactions } \\
\text { involving non-duty free recorded } \\
\text { imports } \\
\text { 5. Value of recorded imports where } \\
\text { customs value was assessed using } \\
\text { transaction prices, breaking out raw } \\
\text { diamond imports; } \\
6 . \text { Value of non-duty free recorded } \\
\text { imports where customs value was } \\
\text { assessed using transaction prices; } \\
\text { 7. Number of transactions involving } \\
\text { recorded imports where customs value } \\
\text { was assessed using transaction prices; } \\
\text { 8. Number of transactions involving } \\
\text { non duty free recorded imports where } \\
\text { customs value was assessed using } \\
\text { transaction prices }\end{array}$ & Quarterly & $\begin{array}{l}\text { Within } 30 \text { days of } \\
\text { the end of each } \\
\text { quarter }\end{array}$ \\
\hline
\end{tabular}


Table 1. Armenia: (Program) Exchange Rates of the CBA (As of December 31, 2008 for dollars per currency rates)

\begin{tabular}{lcc}
\hline & $\begin{array}{c}\text { Drams } \\
\text { Per } \\
\text { Currency }\end{array}$ & $\begin{array}{c}\text { Dollars } \\
\text { Per } \\
\text { Currency }\end{array}$ \\
\hline Australian dollar & 266.57 & 0.6924 \\
Canadian dollar & 313.39 & 0.8140 \\
Swiss franc & 365.58 & 0.9496 \\
Danish krone & 73.26 & 0.1903 \\
Euro & 546.00 & 1.4182 \\
Pound sterling & 558.68 & 1.4511 \\
Japanese yen & 4.27 & 0.0111 \\
Norwegian krone & 55.20 & 0.1434 \\
Russian ruble & 13.13 & 0.0341 \\
Swedish krone & 18.21 & 0.0473 \\
U.S. dollar & {$[\ldots]$} & 1.0000 \\
SDR & 593.00 & 1.5403
\end{tabular}

Table 2. Armenia: KfW and IBRD SME Loan Disbursements 1/ (In billions of dram)

\begin{tabular}{|c|c|c|c|}
\hline \multicolumn{4}{|c|}{2009} \\
\hline March & June & September & December \\
\hline 2.39 & 2.39 & 33.63 & 36.01 \\
\hline
\end{tabular}

1/ Cumulative from December 2008, at program exchange rates. 


\begin{tabular}{|c|c|c|c|}
\hline \begin{tabular}{|} 
Table 3. Armenia: External Financing to the Public Sector (Program) 1/ \\
(in billions of drams)
\end{tabular} \\
\hline \multicolumn{5}{|c|}{2009} \\
\hline March & June & September & December \\
\hline \multicolumn{5}{|c|}{ Project financing } \\
\hline 14.72 & 50.82 & 81.21 & 105.58 \\
\hline 3.09 & $78.93 \quad$ & 97.34 & 16.23 \\
\hline \multicolumn{5}{|c|}{ Rudget support } \\
\hline \multicolumn{5}{|c|}{ Total } \\
\hline 17.81 & $192.50 \quad 02.50$ \\
\hline
\end{tabular}

1/ Cumulative from December 2008, at program exchange rates. 


\title{
INTERNATIONAL MONETARY FUND
}

\author{
REPUBLIC OF ARMENIA
}

First Review Under the Stand-By Arrangement, Requests for Augmentation, Rephasing of Purchases, Waiver of the Nonobservance of Performance Criteria, and Modification of Performance Criteria

\section{Informational Annex}

Prepared by the Middle East and Central Asia Department

June 12, 2009

Contents

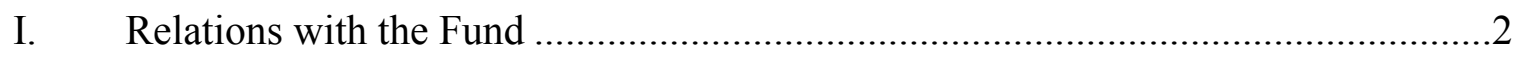

II. Relations with the World Bank ........................................................................6

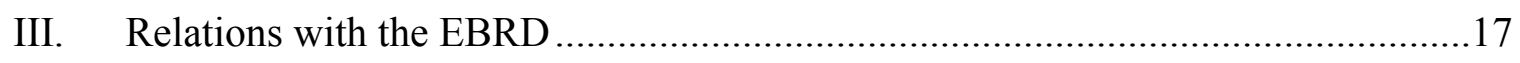

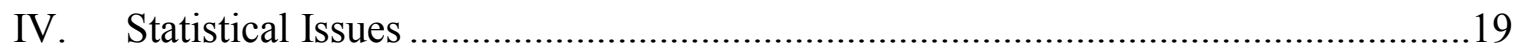




\section{ANNEX I. ARMENia: RElations With THE FUND}

(As of April 30, 2009)

I. Membership Status: Joined 05/28/1992; Article VIII

II. General Resources Account:

Quota

Fund holdings of currency

III. SDR Department:

Holdings

IV. Outstanding Purchases and Loans:

Stand-by Arrangements

PRGF arrangements

V. Latest Financial Arrangements:
SDR Million

92.00

253.56

SDR Million

2.90

SDR Million

161.55

82.40
Percent of Quota

100.00

275.61

Percent of Allocation

n.a.

$\begin{array}{lcccc}\text { Type } & \begin{array}{c}\text { Approval } \\ \text { Date }\end{array} & \begin{array}{c}\text { Expiration } \\ \text { Date }\end{array} & \begin{array}{c}\text { Amount Approved } \\ \text { (SDR Million) }\end{array} & \begin{array}{c}\text { Amount Drawn } \\ \text { (SDR Million) }\end{array} \\ \text { Stand-By } & 03 / 6 / 09 & 07 / 5 / 11 & \frac{368.00}{161.55} & 1.31 \\ \text { PRGF } & 11 / 17 / 2008 & 03 / 6 / 2009 & 9.20 & 23.00 \\ \text { PRGF } & 05 / 25 / 2005 & 05 / 24 / 2008 & 23.00 & \end{array}$

VI. Projected Payments to Fund ${ }^{1 /}$

(SDR Million; based on existing use of resources and present holdings of SDRs)

\begin{tabular}{|c|c|c|c|c|c|}
\hline & \multicolumn{5}{|c|}{ Forthcoming } \\
\hline & 2009 & 2010 & 2011 & 2012 & 2013 \\
\hline Principal & 10.09 & 14.13 & 14.44 & 75.33 & 91.84 \\
\hline Charges/interest & $\underline{1.91}$ & 2.64 & 2.57 & 2.35 & $\underline{1.26}$ \\
\hline Total & 12.00 & 16.77 & 17.01 & 77.68 & 93.10 \\
\hline
\end{tabular}

${ }^{1 /}$ When a member has overdue financial obligations outstanding for more than three months, the amount of such arrears will be shown in this section.

\section{Safeguards Assessment}

Under the Fund's Safeguards Assessments policy, an update safeguards assessment of the Central Bank of Armenia (CBA) was finalized on May 4, 2009, with respect to the PRGF approved on November 18, 2008. The update found that the CBA's safeguards framework has been strengthened since the 2005 assessment in a number of areas. 


\section{Exchange Rate Arrangement}

(a) The de jure arrangement is a "managed float." The de facto arrangement was reclassified to "stabilized (peg-like) arrangement" as of July 1, 2008 from a "managed float." The official exchange rate is quoted daily as a weighted average of the previous day's interbank exchange rates.

(b) Armenia maintains no exchange restrictions on the making of payments and transfers for current international transactions except for exchange restrictions maintained for security reasons, and notified to the Fund pursuant to Executive Board Decision No. 144-(52/51).

\section{Article IV Consultations}

The 2008 Article IV consultation with Armenia was concluded on November 17, 2008. Armenia is subject to a 24-month consultation cycle.

\section{FSAP Participation and ROSCs}

A joint World Bank-International Monetary Fund mission assessed Armenia's financial sector as part of the Financial Sector Assessment Program (FSAP) update during February 16-March 4, 2005. The Financial Sector Stability Assessment (FSSA) report was discussed by the Executive Board on May 25, 2005.

\section{ROSC Modules}

\begin{tabular}{llll}
\hline Standard & Timing & $\begin{array}{l}\text { Publication } \\
\text { Status }\end{array}$ & Document Number \\
\hline $\begin{array}{l}\text { Basel Core Principles for Effective Banking } \\
\text { Supervision (BCP) }\end{array}$ & April 2001 & Unpublished & $\ldots$ \\
$\begin{array}{l}\text { Core Principles for Systemically Important } \\
\text { Payments Systems (CPSS) }\end{array}$ & April 2001 & Unpublished & $\ldots$ \\
$\begin{array}{l}\text { Insurance Principles set by the International } \\
\text { Association of Insurance Supervisors (IAIS) }\end{array}$ & 2001 & Unpublished & $\ldots$ \\
$\begin{array}{l}\text { Principles set by the International } \\
\text { Organization of Securities Commissions }\end{array}$ & 2001 & Unpublished & $\ldots$ \\
$\begin{array}{l}\text { IOSCO) } \\
\text { Code of Good Practices in Monetary and }\end{array}$ & April 2001 & Unpublished & $\ldots$ \\
$\begin{array}{l}\text { Financial Policy Transparency (MFPT) } \\
\text { Code of Good Practices on Fiscal }\end{array}$ & March 2001 & Published & $02 / 37$ \\
$\begin{array}{l}\text { Transparency } \\
\text { Data ROSC module }\end{array}$ & September 2000 & Published & $02 / 06$
\end{tabular}




$\begin{array}{llll}\text { AML-CFT assessment by MONEYVAL } & \text { July 2004 } & \text { Unpublished } & \ldots \\ \begin{array}{l}\text { Basel Core Principles for Effective Banking } \\ \text { Supervision (BCP) update }\end{array} & \text { May 2005 } & \text { Unpublished } & \ldots \\ \text { Corporate Governance } & \text { May 2005 } & \text { Unpublished } & \ldots \\ \text { Data ROSC module } & \text { April 2008 } & \text { Published } & 09 / 50\end{array}$

\section{Resident Representatives}

Ms. Nienke Oomes, since August 2006.

\section{Technical Assistance}

The following table summarizes the Fund's technical assistance to Armenia since 2002.

Armenia: Technical Assistance from the Fund, 2002-09

Subject
Fiscal Affairs Department (FAD)

Tax policy and administration

Tax policy

Tax administration

Public expenditure management

advisor

Tax administration

Public financial management reform

Tax administration

Tax administration

Tax administration

Tax administration

Tax administration

Tax administration
Short-term

Short-term

Short-term

Short-term

Short-term

Short-term

Short-term

Short-term

Short-term

Short-term

Short-term
September 17-October 2, 2003

April 22-May 6, 2004

July 13-27, 2004

November 7, 2003-

November 6, 2004

October 5-18, 2006

November 27 -

December 8, 2006

October 31 - November 13, 2007

January $5-22,2008$

April 1-12, 2008

June 11-17, 2008

February 2-27, 2009

April 28 - May 22, 2009
Counterpart

MFE, State Tax Service (STS), and Customs Committee

MFE, STS, and Customs Committee

MFE, STS, and Customs Committee

MFE, STS

MFE

MFE, STS

MFE, STS

MFE, STS

MFE, STS

MFE, STS

MFE, STS

\section{Legal Department}


Tax legislation

Legislation Development \& FIU

Short-term

Unified Tax Code
September 1, 2004-

December 31, 2005

July 31-August 7, 2006

June 16-23, $2007 \quad$ MFE

\section{Monetary and Capital Markets Department}

Banking system issues
Banking system, deposit insurance,
foreign exchange market development,
and CBA monetary operations.
Unified financial supervision,
mortgage financing markets and
inflation targeting
Financial sector assessment program
update

Recapitalization of the Central Bank of Short-term Armenia

Monetary policy and markets

Strengthening the implementation of monetary policy

Monetary policy implementation and money market development

AML/CFT preventive measures

Inflation Targeting, Foreign Exchange

Market Development and Responding

to Dedollarization

Exchange rate and collateral

\section{Statistics Department}

Data dissemination standards

Short-term

Balance of payments: remittances

Short-term

Short-term

Short-term

Short-term

Short-term

Short-term

Short-term

Short-term

Short-term

\section{International Capital Market Department}

Sovereign credit quality

Short-term

Sovereign credit risk

Short-term
April 15-19, 2002

CBA

June 24-July 4, 2002

CBA

January 26-February 6 2004

February 16-

March 4, 2005

December 8-14, 2005

CBA

December 4, 2005-

December 31, 2006

March 8-21, 2006

CBA

October 5-17, $2006 \quad$ CBA

April 1, 2006-April 30, 2007

August 29-September 10,2007

February $23-27,2009$

CBA

September 18-25, $2003 \quad$ National Statistical Service

August 22-September CBA 5, 2006

September 6-10, 2005

CBA

August 20, 2005-

CBA 


\section{ANNEX II. ARMENIA: RELATIONS WITH THE WORLD BANK}

(June 10, 2009)

Country Director: Asad Alam

Telephone: 99532913096

\section{IMPLEMENTATION OF STRUCTURAL REFORM MEASURES}

\section{A. Business Environment}

1. The World Bank Group has supported the Government in making progress in removing administrative barriers for business and investment and has strengthened the consultative mechanisms with the business community, though overall there is still much further work remaining to improve Armenia's competitiveness. Recent steps taken include: consolidating, downsizing, and clarifying mandates of various inspections; enacting a new law on and reducing the costs of business registration; streamlining licensing procedures; issuing new accounting recommendations for small and medium-sized enterprises; establishing a regulatory framework that allows privatization of urban land by business entities; and adopting simplified procedures for obtaining site development and construction permits. The capacity of the Armenian Development Agency as a focal point for Government's efforts to promote investment and exports as well as for identifying the remaining bottlenecks in the business environment has been strengthened. The functioning of the Business Council has improved and the private sector's awareness of its activities has been enhanced.

2. Recent business surveys of Armenian entrepreneurs suggest that these efforts have resulted in a more positive private sector perception of the business and investment environment. For example, the average time necessary to get construction and building renovation permits was reduced from 310 days in 2001 to 112 days in 2006. Despite these improvements, however, there is still considerable scope for further reforms in the areas of competition and strengthening of the business and investment climate. These reforms are supported by the proposed Development Policy Operation (DPO) series (2009-2011).

\section{B. Public Sector Reform}

3. Further strengthening of tax and customs administration was at the core of the Poverty Reduction Support Credit (PRSC) series, and remains at the core of the new proposed DPO series, as much work remains to reduce the burden imposed on firms and to raise revenue as a share of GDP. The shortcomings of the tax and customs administrations remain acute: recent survey data show tax and customs as one of the main constraints affecting the operations and growth of Armenian companies. One of the major reforms was the adoption of a self-declaration system. A separate division for taxpayer service has been established within the SRC headquarters designated for: (i) defining the policies and 
procedures of taxpayer education and assistance for tax inspectorates; (ii) overseeing the performance of taxpayer service divisions of tax inspectorates; and (iii) managing the taxpayer education and assistance operations throughout the State Revenue Committee (SRC). A work plan for improving the enforcement of the Law on Declaring Individuals' Property and Income was also developed. Recording commissions were abolished as of July $1^{\text {st }} 2008$.

4. Over the PRSC program, some significant structural improvements were achieved in customs administration, such as the introduction of modern software and improved personnel practices. The central reform was the institution of direct trader input (DTI) that currently covers about 70 percent of imports. DTI has initiated a systemic change away from the intimate involvement of customs officials at the declaration validation stage, where opportunities for discretionary behavior are rife. The system has the capability to provide for immediate validation and assessment of acceptable declarations. Moreover, customs introduced a self declaration system and reduced the role of reference prices. It has also provided public access to customs values through the official customs website (www.customs.am). Legislative amendments for the implementation of bank guarantees were approved by the government at end-September 2007. Progress has also been made in selectivity of customs control. However, these efforts are still too fragmented to meet the challenges of a modern customs administration.

5. The SRC Action Plan, recently issued by Prime Ministerial Decree, agreed by the Government and the Bank under the DPO proposes a set of actions that would lead to significant improvement in both tax and customs. The SRC Tax Service reform program focuses on strengthening compliance by improving audit and enforcement functions, strengthening taxpayer services via the Large Taxpayer Office and a one stop shop for taxpayers, electronic filing, and improved back office functions. On the Customs side, the DPO-supported program proposes the following actions: in DPO-2 the introduction of a "green channel by default" approach to clearance, including an overhaul of the clearance and examination policy; and reinforcing the preventive and enforcement wing to fight smuggling at the borders and inside national territory, support investigations, gather and process intelligence, and contribute to the agency's enforcement activities related to cross-border and other international commercial activities. All these reform measures have been adopted by the Government in an action plan with a timeline and key performance indicators, with budget commitment for adequate funding from 2009-2011, and a costing to be done by December 2009.

6. The World Bank has also been supporting the government in a wide range of public administration reforms, in particular in developing the anti-corruption strategy, improving policy formulation, and strengthening the civil service. Ongoing support from the Bank has already assisted the Government in advancing merit based recruitment and performance management in the civil and municipal services, capacity building for certification, and 
external audit reforms. Information management systems have been delivered for central and municipal administration. The electronic document management system, piloted in the government staff and two pilot ministries, is already being rolled-out across the entire government. Development of e-tendering modules for planned electronic public procurement system is also underway.

7. In order to strengthen conflict of interest management in the public sector, the Government also recently submitted to the National Assembly, in the context of DPO-1, the Law on the Public Service, which includes conflict of interest provisions necessary to ensure the framework for further implementation. The Government's objective is to increasingly implement conflict of interest regulations and strengthen public service ethics through a more robust legal framework and publicly available monitoring reports, including on asset disclosure.

8. The Bank has also supported Armenia's public financial management (PFM) reform agenda by strengthening external audit through the Chamber of Control, assisting development of a modern internal audit capacity, and building capacity in local government. Since 2003 there has been continuous support to capacity building for treasury reforms, which in particular produced technical and functional specifications for a Government Financial Management Information System. Further, the Bank supported the government in preparation of its Public Expenditure and Financial Accountability (PEFA) Performance Assessment Report, which was published in October 2008. The conducted and ongoing analytical and capacity building work will shape the PFM system as Armenia prepares its PFM reform strategy.

\section{Legal and Judicial Reform}

9. The World Bank has supported the Government of Armenia to establish the core legal framework necessary for private sector operations, including the civil procedures code, the procurement law, the business registration law and the public auction law. The fully restructured and enacted bankruptcy law is now harmonized with the civil code and the civil procedures code, and strengthens the enforcement mechanisms for bankruptcy procedures. The Government also has made significant progress in drafting the necessary legislation to improve the lending environment through strengthening the procedures for collateral registration and for foreclosure and enhancing the knowledge of the judiciary concerning commercial contracts.

10. With support from the Second Judicial Reform Project (JRP2), the judiciary and the Government formulated a long term strategy, which is reflected in the judicial code and consequent establishment of the Judicial Department responsible for judicial administration and a career judicial service (excluding judges). With the support of JRP1 all the normative legal acts were made available electronically through the ARLIS legal database with free on- 
line access. Special automated case management information systems were developed and piloted for courts (CAST) and the Enforcement Service Department. Under JRP2 CAST now has been rolled-out across the entire judicial system. The World Bank has also supported the rehabilitation of 13 courthouses housing 15 courts and the installation of digital case recording audio systems in all renovated or new courtrooms. Additional courthouses throughout Armenia are under construction as part of JRP2.

\section{Energy and Infrastructure}

11. Supported by the World Bank, the Government and the regulator have also made satisfactory efforts to improve the legal and regulatory framework in the energy sector in order to establish a supportive environment for the private operators. The market rules in the energy sector have improved. The Electricity Distribution Company is allowed to enter into direct contracts with the electricity generators and service providers, which has enhanced sector transparency. The regulator has adopted and enforced service quality standards for electricity supply. Despite this satisfactory performance, continued efforts are crucial for improvements in the energy sector through restructuring the midstream companies and strengthening the regulatory framework to ensure adequate functioning, transparency, and reliability.

12. In order to remove the obstacles for realization of energy efficiency potential, the Government is committed to developing for DPO-2 a time-bound energy efficiency action plan with efficiency targets, and designating a responsible entity with adequate resources. Specific measures will address the following key issues: adjustment in energy sector utilities' regulatory framework to ensure that utilities and their customers make energy production, distribution, and consumption decisions that increase the efficiency of energy utilization; designation and empowerment of an energy efficiency entity, allocation of financial resources to sustain its operation, and strengthening of its capacity; and development of incentives and standards to promote import, manufacturing, and use of energy efficient equipment.

13. There has been progress in improving fiscal discipline and reducing losses in the irrigation and water sectors. The World Bank has been working with the Government to: (i) upgrade the management capacity of public companies in these sectors; (ii) ensure a gradual increase in tariffs to cost recovery; (iii) provide additional investments to improve technical efficiency; and (iv) ensure that the budget provides adequate financing for water consumed by public sector entities. The World Bank has also supported an innovative publicprivate partnership in water supply, which has substantially increased reliable water supply throughout the country.

14. There has been substantial progress made with the liberalization of the telecommunication sector and adoption of pro-competitive regulations. Nevertheless, 
important constraints continue to remain, including sub-optimal legal and institutional frameworks for efficient planning, allocation, and monitoring of radio spectrum; unaddressed issues of interconnection regime, provision of open access, infrastructure sharing, and local loop unbundling. As part of the DPO-supported program, the Government is addressing these constraints.

15. Current mining sector law and regulations are conflicting and overlapping, the fiscal regime is onerous, and the regulatory and institutional set-up lacks transparency and public accountability. Through the DPO, the Government is developing a new mining code, which provides the legal foundation for an appropriate fiscal, environmental, social, and licensing regime.

\section{E. Rural Development, Agriculture, and Environment}

16. Recent World Bank support for the rural development and agriculture sector has largely been through the Rural Enterprise and Small-scale Commercial Agriculture Development Project (RESCAD). The objective of this project is to support the development of Armenia's small and medium-scale rural businesses by: (a) improving the ability of farmers and rural entrepreneurs to access markets; and (b) stimulating market-oriented private and public investments in rural areas. It helped provide long term credit resources for rural agri-business, rural enterprises, and farmers. It also helped introduce, test, and demonstrate technical innovations, and focused on processing and marketing of agricultural production through a series of competitive small grant. It helped strengthen extension services to deliver technical advice to farmers and rural businesses. The project also supported a modern legal and regulatory framework for production of high generation seed in support of private seed production activities. In addition, the Bank supported irrigation development and dam safety projects centered on: (a) introducing participatory irrigation management practices; and (b) returning a larger amount of land to irrigation. Water User Associations (WUAs) cover all irrigated land in Armenia and there is anecdotal evidence of improvements in crop yields. The Avian Influenza Preparedness project, which was launched in 2006 to help Armenia address the Avian Flu emergency, then restructured to focus on broader animal and human health issues, and is helping Armenia to prepare for disease emergencies.

17. The Bank has also supported analytical work on the rural sector, including on marketing and value chains for various products, and sector-specific development challenges, including in-depth analysis of food safety and agricultural health issues. Considerable support has also been mobilized for the forestry sector through investment lending and the PRSC series, which promoted substantial legal and institutional reforms, including sustainable forest management through enhanced control of illegal logging and community forest management. 
18. Going forward the Government is also committed to strengthening environmental impact assessment (EIA) legislation as part of the DPO series. Introducing strategic environmental assessment and environmental impact valuation would help enhance transparency. Improved EIA legislation would help enhance transparency and accountability in policy decisions, and promote stakeholder participation in EIA at the planning stage. The Government also sees the need to address damages caused by mining activities by enforcing regulations more effectively.

\section{F. Education and Health}

19. The World Bank has provided significant support for reforms in education and health. Progress has been made with regard to education sector financing and management. The Government adopted a medium-term action plan for improving the financial management, accounting, and financial reporting for higher education institutions. Accountants at the higher institutions have been trained and special software has been prepared. The Government also increased the state budget allocation for primary and secondary education, while improving teachers' salaries. In addition, the Government approved a strategy on preschool education and prepared a pilot project for its implementation in two marzes.

20. Despite these important improvements, there is scope for further reforms, including:

(i) further refining the curriculum to enhance relevance and reduce the overload, particularly in upper secondary education; (ii) strengthening the teacher education and ongoing professional development strategy to make it more demand-driven and school-based; (iii) enhancing capacities for ICT use and integration in teaching and learning, (iv) addressing governance and transparency issues in higher education; (v) enhancing standards for higher education in response to market demands; (vi) developing a student loan system; (vii) promoting school readiness and equal opportunities through an improved preschool education system and increasing the enrollment rate; and (viii) strengthening monitoring and financial reporting of the noncommercial organizations (NCOs) in the education sector. The Second Education Quality and Relevance Project, approved by the Board of the World Bank in May 2009, will support most of these second-generation reforms in the sector. Moreover, the DPO-related measures will focus on preschool and higher education, heretofore the two neglected sub-sectors in the education system.

21. The Bank also supported the Government's reform program in the health sector. The Government undertook measures to increase financing of primary health care in order to secure access to quality basic health services, in particular for the poor and in rural areas. An important element was the decision to introduce further reforms in the Basic Benefits Package. The Government strategy for increasing revenues in the short term is to keep the existing benefits package but raise reimbursement rates to reduce the gap resulting from higher service costs, while introducing an official co-payment policy to address informal payments. Further reforms are needed to improve the population's overall health status, 
reduce child and maternal mortality, increase the use of the healthcare system by rural and low-income groups, monitor public health and promote better health behavior, and strengthen monitoring and financial reporting of NCOs. The Government is also developing a national strategy on combating non-communicable diseases (NCDs) through development and implementation of specific NCD priority programs and allocation of adequate public resources. Both the copayments policy reforms and the strengthening of NCD management are supported by the DPO series.

22. The Government has also reformed the hospital sector by consolidating twenty-four public hospitals and thirteen outpatient health care institutions. A Bank-financed project supported upgrading hospitals' physical condition and equipment, while also improving internal management and governance of the three hospital mergers in Yerevan, which demonstrated the greatest efficiency gains. In addition, the Government adopted a regional hospital optimization/modernization plan aimed at improving the efficiency and productivity of outpatient services in the regions. The consolidation of regional hospitals in smaller networks is underway.

23. The medium-term financial management action plan for public hospitals was adopted by the Government to prepare new reporting and accounting procedures, including cost accounting manuals. All public hospitals use updated financial management and accounting procedures. The Government plans to conduct independent financial audits of all public hospitals starting in 2009.

\section{G. Social Protection and Insurance}

24. Since 1999, the Government has been replacing a range of fragmented cash and noncash benefits and privileges with better-targeted transfers to families. The Government has been supported by the World Bank to complete several important steps to enhance its capacity for administration of transfers to families, including: (i) re-registration of poverty benefit recipients; (ii) beneficiary assessment of existing benefits; and (iii) establishment of a central database for poverty benefit recipients. Data from recent household surveys suggest that the system of benefits and transfers to the poor has become an efficient instrument for reducing extreme poverty.

25. In the context of the economic crisis, and with the support of DPO-1, the Government has increased funding for social safety net programs, including Family Benefits (FB) and pensions in the 2009 budget law (relative to 2008), increased funding for unemployment insurance (based on actual numbers of unemployed), and protected these programs from any reductions. Furthermore, the Government has restored funding for selected priority programs for the poor and vulnerable in health, education, and agriculture in accordance with the original 2009 budget. At the same time, the Government has undertaken a review of FB program beneficiaries and excluded non-eligible households to improve targeting efficiency; 
FB budget savings are being used to expand program coverage of the poor. Further improvements in targeting efficiency will be supported over 2010-2011 by the DPO program.

26. The Government of Armenia approved a new pension strategy in November 2008 along with an action plan for implementation. The strategy includes the mandatory introduction of a second pillar for those born after January 1, 1970 (financed by an additional contribution), a flat-benefit budget-financed first pillar, and a universal zero pillar for those who did not contribute to the system. A task force has been established to monitor the implementation of the pension reforms, and the Government has requested the Bank's support.

\section{World Bank Group Strategy AND Portfolio}

27. A new World Bank Group Country Partnership Strategy (CPS) was prepared for Armenia in the context of the global economic crisis and its impact on the country. It focuses on the near term needs of addressing vulnerability and mitigating the adverse poverty effects of the crises as well as laying the foundation for promoting medium term competitiveness and growth. The CPS will be discussed by the Board of Directors on June 11, 2009. The central challenge for the Government is to mitigate the economic and social impacts of the current economic crisis, which are likely to persist for several years, while also laying the foundations for rapid post-crisis recovery and growth. With limited domestic resources, the primary short-term focus has to be to address current crisis needs and seek to leverage donor funding to support a modest fiscal stimulus, boost domestic investments, especially for job creation, and scale up targeted social safety net programs. At the same time, being a small open economy, Armenia's future is inextricably linked to that of the global economy and its best strategy is to be prepared to take full advantage of the eventual global recovery.

28. Several principles will consequently guide the proposed CPS. First, there is direct alignment with Armenia's national Sustainable Development Program which is being revised to take account of the current international conditions. In particular, Bank strategy will seek to address the urgent while staying focused on the important medium-term issues. Second, the Bank Group must exercise selectivity and progressively disengage from areas where reform or investment needs have been substantially satisfied or where other donors can lead, or where the country has adequate internal knowledge and capacity. Third, the Bank Group will increasingly rely upon leveraging other development partners given competing demands on IBRD resources and the regional administrative budget. Fourth, the CPS responds to the government's request to front-load IDA and IBRD resources over FY09-10, consistent with existing rules, in order to respond promptly to the effects of the international crisis.

29. IDA country resource envelopes are determined annually using the Performance Based Allocation (PBA) methodology common to all IDA countries. The PBA draws on the Country Policy and Institutional Assessments and performance of the ongoing portfolio. 
Armenia has a very strong PBA rating and as a consequence is eligible for about US\$50-60 million equivalent per year. In addition, Armenia is now a blend country and is IBRDeligible.

30. The principal purpose of the new proposed Development Policy Operation (DPO) program (2009-2011) is to provide support to the Government of the Republic of Armenia to address vulnerabilities, particularly in the context of the global economic crisis, while strengthening competitiveness for more rapid growth in the post-crisis period. To mitigate vulnerability the operation will support the Government in managing the impact of the global crisis by maintaining a sound macroeconomic and fiscal framework while protecting the poor; and strengthening the management of education, health, and social protection programs. To strengthen competitiveness for recovery and growth, the operation will assist the Government in improving economic competition and the business climate; strengthening the regulatory environment in infrastructure; improving competitiveness through the sustainable use of energy and natural resources; and improving public sector efficiency and effectiveness. The First DPO is scheduled for Board presentation on July 2, 2009.

31. World Bank lending to Armenia as of June 1, 2009 totals US\$ 1,187 million, (including 2 GEF operations), of which US\$ 1,042 million has been disbursed. The current Bank portfolio consists of 17 IDA credits, one IBRD loan, one Geofund operation, and one GEF project, for a total commitment of US\$ 379.9 million, of which US\$ 199 million is disbursed. The Armenia portfolio continues to be low risk with all projects rated in the satisfactory range. In FY09, the following projects were approved: Additional Financing for Municipal Water Project (US\$ $20 \mathrm{mln}$ ), three IDA Fast Track Facility operations, including the Lifeline Roads Improvement Project (US\$ $25 \mathrm{mln}$ ) and additional financing for the Social Investment Fund project (US\$ $8 \mathrm{mln}$ ), and for the Rural Enterprise and Small-scale Commercial Agriculture Development Project (US\$ $2 \mathrm{mln}$ ), the first IBRD operation Access to Finance for Small and Medium Enterprise (US\$ $50 \mathrm{mln}$ ), a Geofund operation (US\$ $1.5 \mathrm{mln}$ ), and the Second Education Quality and Relevance Project (US\$ $25 \mathrm{mln}$ ).

32. The IFC is active in Armenia and has a committed portfolio of US\$ 36.7 million, as of April 2009. In the short term, the IFC, together with the Bank, is focused on addressing the challenges of the crisis. In particular, the IFC is providing short-term liquidity, including trade finance support, to Armenian banks, and stands ready to support the banks' capital with equity or quasi-equity. The IFC has also refocused its advisory services program to help banks with loan servicing and portfolio management. At the same time, the IFC will continue to invest and provide advisory services to support longer term goals, including access to longer term finance, improvement of the business climate, and increased competitiveness. 
List of World Bank Lending to Armenia, June 2009

(In millions of U.S. dollars)

\begin{tabular}{|c|c|c|c|c|c|}
\hline & Active Projects & Credit Amount & Disbursement & $\begin{array}{l}\text { Approval } \\
\text { Date }\end{array}$ & Closing Date \\
\hline \multicolumn{2}{|c|}{ Active Projects } & 379.9 & 199.11 & & \\
\hline 1. & Irrigation Dam Safety & 26.6 & 24,82 & 06/24/99 & 09/30/09 \\
\hline 2. & Social Protection Admin. & 5.15 & 5,03 & 06/10/04 & $12 / 31 / 09$ \\
\hline 3. & $\begin{array}{l}\text { Educ. Qual. \& Relevance (APL } \\
\# 1)\end{array}$ & 19.0 & 17,94 & $01 / 20 / 04$ & $11 / 30 / 09$ \\
\hline 4. & Municipal Water and WW & 43.0 & 23,47 & 05/04/04 & $12 / 31 / 11$ \\
\hline 5. & Health System Modernization & 19.0 & 16,95 & 06/10/04 & $06 / 30 / 10$ \\
\hline 6. & Irrigation Dam Safety 2 & 6.75 & 6,28 & 06/10/04 & $12 / 31 / 09$ \\
\hline 7. & Public Sector Modernization & 10.15 & 8,64 & 05/04/04 & $06 / 30 / 10$ \\
\hline 8. & $\begin{array}{l}\text { Rural Enterprise \& Small Scale } \\
\text { Agric. }\end{array}$ & 22.0 & 19,07 & 07/07/05 & $05 / 31 / 10$ \\
\hline 9. & Urban Heating & 15.0 & 12,43 & $07 / 12 / 05$ & $06 / 30 / 10$ \\
\hline 10. & Yerevan Water/Wastewater & 20.0 & 8,60 & $02 / 24 / 05$ & $02 / 28 / 11$ \\
\hline \multirow[t]{2}{*}{11.} & Renewable Energy & 5.0 & 4,67 & 03/29/06 & $12 / 31 / 10$ \\
\hline & Renewable Energy (GEF) & 3.0 & 1.83 & 03/29/06 & $12 / 31 / 10$ \\
\hline 12. & Avian Influenza Preparedness & 6.25 & 4,04 & $06 / 02 / 06$ & $07 / 31 / 10$ \\
\hline 13. & Armenia SIF III & 33.0 & $\begin{array}{c}20,88 \\
2,42\end{array}$ & $10 / 26 / 06$ & 06/30/11 \\
\hline & $\begin{array}{l}\text { Health Systems Modernization } \\
\text { (APL-II) }\end{array}$ & 22.0 & 4,18 & 03/08/07 & $12 / 31 / 12$ \\
\hline 15. & Judicial Reform II & 22.5 & 10,23 & 03/08/07 & $12 / 31 / 12$ \\
\hline & Access to Finance for SME & 50.0 & 0.13 & 02/24/09 & $09 / 28 / 11$ \\
\hline & $\begin{array}{l}\text { Lifeline Roads Improvement } \\
\text { Project }\end{array}$ & 25.0 & 7,50 & 02/24/09 & $12 / 31 / 10$ \\
\hline 18. & Geofund 2: Arm & 1.5 & - & $02 / 24 / 09$ & $04 / 30 / 11$ \\
\hline & $\begin{array}{l}\text { Second Educ. Qual. \& Relevance } \\
\text { (APL \#2) }\end{array}$ & 25.0 & - & 05/12/09 & $11 / 30 / 14$ \\
\hline \multicolumn{2}{|c|}{ Closed Projects } & 807.8 & 845.8 & & \\
\hline & Irrigation Development & 29.9 & 34.5 & 08/30/01 & 03/31/09 \\
\hline & Natural Resource Management & 8.3 & 9.4 & 06/04/02 & 01/31/09 \\
\hline & $\begin{array}{l}\text { Natural Resource Management } \\
\text { (GEF) }\end{array}$ & 5.1 & 4.0 & $06 / 04 / 02$ & 01/31/09 \\
\hline & Economic Rehabilitation & 60.0 & 57.6 & $02 / 28 / 95$ & $06 / 30 / 96$ \\
\hline & SAC & 60.0 & 56.9 & $02 / 29 / 96$ & $12 / 31 / 97$ \\
\hline & Institution Building & 12.0 & 10.6 & $03 / 30 / 93$ & $11 / 30 / 97$ \\
\hline & Earthquake Rehabilitation & 28.0 & 29.7 & 02/01/94 & $06 / 30 / 97$ \\
\hline & Power Maintenance & 13.7 & 12.8 & $12 / 08 / 94$ & 06/30/99 \\
\hline & SAC II & 60.0 & 63.1 & $08 / 26 / 97$ & 06/30/99 \\
\hline & SATAC I & 3.8 & 2.9 & $02 / 29 / 96$ & $06 / 30 / 00$ \\
\hline 29. & Highway & 31.0 & 29.9 & $09 / 14 / 95$ & $12 / 31 / 00$ \\
\hline & Social Investment Fund & 12.0 & 11.4 & $11 / 09 / 95$ & $12 / 31 / 00$ \\
\hline & Irrigation Rehabilitation & 43.0 & 40.9 & $12 / 08 / 94$ & $05 / 31 / 01$ \\
\hline & SAC III & 65.0 & 70.3 & $12 / 22 / 98$ & $06 / 30 / 01$ \\
\hline & Enterprise Development & 16.8 & 16.4 & $12 / 24 / 96$ & 07/01/02 \\
\hline
\end{tabular}


34. SATAC II

35. Education

36. SAC IV

37. Health

38. SAC V

39. Title Registration

40. Transport

41. PRSC I

42. Agric. Reform Support

43. Social Investment Fund II

44. Investment and Export Facilitation

45. Municipal Development

46. PRSC II

47. Enterprise Incubator

48. Judicial Reform

49. Electricity Transmission \& Distribution

50. PRSC III

51. PRSC IV

Total
5.0

15.0

50.0

10.0

40.0

8.0

40.0

20.0

16.3

20.0

1.0

30.0

20.0

5.0

11.4

21.0

28.0

18.5

$1,187.7$

$\begin{array}{ccc}4.9 & 08 / 26 / 97 & 12 / 31 / 02 \\ 16.2 & 11 / 20 / 97 & 10 / 31 / 02 \\ 58.4 & 05 / 22 / 01 & 03 / 31 / 03 \\ 10.4 & 07 / 29 / 97 & 12 / 30 / 03 \\ 45.9 & 03 / 13 / 03 & 06 / 30 / 04 \\ 8.9 & 10 / 13 / 98 & 09 / 30 / 04 \\ 45.5 & 06 / 08 / 00 & 12 / 31 / 04 \\ 20.8 & 18 / 11 / 04 & 12 / 31 / 05 \\ 17.6 & 01 / 27 / 98 & 06 / 30 / 05 \\ 22.7 & 05 / 11 / 00 & 12 / 31 / 05 \\ 1.2 & 04 / 16 / 02 & 12 / 31 / 05 \\ 33.1 & 06 / 11 / 98 & 01 / 31 / 06 \\ 21.5 & 01 / 19 / 06 & 06 / 30 / 07 \\ 5.7 & 11 / 30 / 01 & \\ & & 12 / 31 / 06\end{array}$

$13.0 \quad 09 / 14 / 00$

$12 / 31 / 06$

$22.8 \quad 03 / 04 / 99$

06/30/07

$28.5 \quad 03 / 08 / 07$

$18.3 \quad 11 / 27 / 07$

06/30/08

06/30/08 


\title{
ANNEX III. ARMENIA: RELATIONS WITH THE EUROPEAN BANK FOR RECONSTRUCTION AND DEVELOPMENT (EBRD)
}

\author{
(As of May 2009)
}

1. As of May, 2009, the EBRD approved 74 projects in the power, transport, agribusiness, municipal and infrastructure, property, construction and financial sectors. Total commitments amounted to around EUR 293 million.

2. There are three sovereign projects. First, the EBRD approved a sovereign guaranteed loan of EUR 54.8 million for construction of the Hrazdan Unit 5 thermal power plant in March 1993, partly aimed at the eventual closure of Armenia's nuclear plant in Medzamor. The government was contemplating the privatization of Hrazdan Unit 5 as the completion of this plant was constrained by limited budgetary resources. The EBRD had funded technical assistance for the Hrazdan privatization prospectus and followed the privatization process. The Hrazdan Thermal Power Complex was transferred to the Russian Federation in the context of the debt-for-equity deal. Second, in November 1994, the agreement on a EUR 21.8 million loan to build an air cargo terminal in Zvartnots airport was signed under a guarantee by the Armenian government. The airport was transferred to private management in 2002 (according to concession agreement). The new management has prepared a master plan for the development of the airport, which is expected to generate further cargo traffic for the cargo terminal. Third, the EBRD approved a 7 million EUR loan to the State Committee for Water Systems, owner of the water and wastewater assets located in the small municipalities outside of Yerevan, in April 2007. The proceeds of this loan will be used to improve wastewater treatment in five municipalities located near Lake Sevan.

3. Most of the Bank's projects in Armenia are in the private sector. Amongst other corporate clients the EBRD has provided a loan to the Yerevan Brandy Company owned by Pernod Ricard of France (EUR 16.5 million). In 2007 the Bank provided a USD 20 million loan to the private concessionaire of Zvartnots International Airport, Armenia's principal gateway. The loan was a commercial facility with no sovereign support. Other private sector finance includes relatively smaller loans to private companies and equity participation in a number of companies in various sectors of the industry. In the banking sector, a first equity participation in the Commercial Bank of Greece-Armenia (EUR 1.1 million) was approved in late 1999 and a second equity participation in Armeconombank was approved in 2004. The Bank also acquired an equity stake in an Armenian non-bank financial intermediary, CIRCO, an insurance subsidiary of Cascade Capital Holding. A Multi-Bank Framework Facility of EUR 10 million, aimed at providing medium to long term funds to Armenian banks was activated in early 2000. In 2006 the Armenian Multi bank Framework Facility II at eth amount of USD 40 million was approved, expanding the scope of eligible projects to equity and nonbank financial institutions financing. Subsequently, due to successful utilization of the facility, it was extended by another USD 80 million. The EBRD expanded its relationship with the partner banks in Armenia from four to ten. Six banks were provided with new credit facilities under the MBFF, including through historic (for Armenia) commercially-syndicated loans to ACBA Credit Agricole Bank and Armeconombank. The Bank acquired new equity stakes in three banks including those with foreign strategic co-investors (ProCredit and Byblos bank Armenia) and Ararat Bank. One institution (Armeconombank) was provided 
with a mortgage facility, and the first leasing facility in Armenia was signed with ACBA leasing. Co-financing facility with local banks was also extended through three MCFF facilities with eleven MCFF sub-loans. By means of MCFF the Bank has entered such new sectors as healthcare and telecom, in addition to significantly expanding its portfolio of agribusiness loans. A Trade Facilitation Program with the purpose to facilitate access of Armenian banks to trade financing was also made available to five Armenian banks. In April 2009, the EBRD signed a EUR 42 mln loan agreement with Electric Networks of Armenia to upgrade and modernization of the obsolete low-voltage infrastructure and improve distribution efficiency.

4. The EBRD has launched the Turn Around Management (TAM) and Business Advisory Service programs in Armenia in 2003, originally funded by the EU-Tacis program but now funded from the ETC Fund, to support micro, small, and medium-sized enterprises. Since 2003 BAS has completed 603 projects in the amount of $€ 2.4$ million, while TAM delivered 17 projects.

5. Supporting development of renewable energy was another core activity of the Bank. To that end, the EBRD joined forces with the WB, USAID, and Cascade Credit (a financing arm of the Armenian-American Cafesdjian Foundation) to launch the Armenian Renewable Energy Programme (AREP). The Bank's participation took the form of a loan to Cascade Credit. The Bank also continued to finance renewable energy projects on its own through Direct Lending Facility, with two such projects signed. In addition to renewable energy, the Bank returned to the mainstream segment of the sector, seeking to support post-privatisation development of the sector with a loan to the Armenian privately-owned power distribution company.

6. Projects identified by the Bank for future development are well-diversified across sectors, and includes several relatively large transactions, thus reflecting economic growth in Armenia and the country's increasing attractiveness to foreign investors. Additional business opportunities are offered by the country's infrastructure sector, including projects in the public sector (municipal and sovereign). Equity transactions through Direct Investment Facility represent one of the fastest growing portfolio segments in Armenia, which has emerged as the leading DIF country in the Caucasus.

7. The EBRD's current country strategy was approved in April 2009. The key priorities of the EBRD for the coming years are: (i) financial sector; (ii) enterprise sector, particularly SME and micro-enterprise financing through credit lines to Armenian banks or direct loans and equity investments, (iii) infrastructure investments in the development of alternative energy sources and municipal infrastructure projects and (iv) portfolio monitoring and implementation support. 


\section{AnNex IV. ARmEnia: Statistical IsSUES}

1. Data provision has some shortcomings, but is broadly adequate for surveillance. Further improvements in real, fiscal, and external sector statistics would be desirable in order to facilitate enhanced design and monitoring of economic policies. The overall quality, timeliness, and coverage of macroeconomic statistics have improved significantly over the past few years. The Fund has substantially facilitated this process through technical assistance from the Statistics Department, the Fiscal Affairs Department, and the Monetary and Capital Markets Department. On November 7, 2003 Armenia subscribed to the Special Data Dissemination Standard (SDDS). The April 2008 data ROSC mission prepared a detailed evaluation of the quality of the macroeconomic statistics.

\section{Real sector statistics}

2. The National Statistics Service (NSS) has made significant changes to the national accounts methodology to bring it in line with best international practices although some shortcomings on data sources and methods remain. Progress has been made in developing estimates of monthly and (constant price) quarterly GDP that are now published. Basic data collection procedures have also partially improved. The national accounts statistics are compiled following the conceptual frameworks of the 1993 SNA and ESA 95. The classification of value added by economic activity follows the ESA 95 directions and data are published grouped accordingly to the A3, A6, A17 and A60 codes of the EU nomenclature of economic activities.

3. Annual and quarterly GDP estimates are compiled at current prices, at comparable previous year's prices, and at average annual prices of the base year (1998) for the series up to the year 2006. Since 2007, GDP at constant prices is computed at average annual 2005 prices. The April 2008 ROSC mission found that compilation techniques for the estimates of GDP by production at constant prices are sound, however there is still need for improvements in the corresponding estimates of GDP by expenditure, particularly regarding the deflators of imports and exports. The mission also found that government expenditures and some transactions with the rest of the world are recorded on a cash basis rather than the required accrual method. Moreover, quarterly data are still collected on a cumulative basis, which are likely to undermine their accuracy. Additionally, statistical techniques need improvements regarding the estimates of the imputed rental services for owner-occupied dwellings, consumption of fixed assets, and work in progress in agriculture.

4. The CPI covers 11 large population centers and the capital city. Since January 2006 the CPI has been computed using 2005 weights. Concepts and definitions used in the compilation of the CPI are broadly in line with international standards; source data and compilation techniques are generally adequate. The NSS compiles a ten-day and a monthly CPI. The ten-day index and the monthly index are disseminated jointly. 


\section{Government finance statistics}

5. The budget execution reporting system compiles data on a cash basis supplemented with monthly reports on arrears and quarterly reports on receivables and payables. Daily revenue and cash expenditure data for the central government are available with a lag of one to two days. The Ministry of Finance (MoF) is undertaking a comprehensive reform of the treasury system, including the introduction of an internal auditing system in line ministries and their budgetary institutions. A single treasury account (TSA) was introduced in 1996, and all bank accounts held by budgetary institutions were closed, except for Project Implementation Units that are required by donors to operate with commercial banks' accounts. Starting in 2002, some budgetary institutions have been converted into "noncommercial organizations" (NCOs). These units have been taken out of the treasury system and have their own bank accounts but report data on cash flows and balances to the MoF since 2003. These exceptions notwithstanding, all government receipts and payments are processed through the TSA, although there are still shortcomings on the timeliness and quality of data on the operations of local governments. Classification of government transactions by function and economic category are generally in line with the Manual on Government Finance Statistics 1986, and monthly data on central government operations are disseminated one month after the reporting period.

6. The budget presentation and the classification of items under the economic and functional classification of expenditures needs to be made more transparent; for instance, the data have been subject to frequent reclassifications, and wages for military personnel are reported in the broader category of "other" goods and services rather than as a wage item. The reconciliation of central government with general government operations is done by the NSS in cooperation with the MoF.

7. Since 2008, government finance statistics meet the classification requirements of the Government Finance Statistics Manual 2001 (GFSM 2001) for central government. The plans for improvement of the Ministry of Finance (MoF) envisage further significant progress within the next two years in implementing the GFSM 2001 classification for local government in 2009 and in accrual recording for all units of general government in 2010.

\section{Monetary and financial statistics}

8. Monetary and financial statistics are provided on a timely basis. Daily data on the accounts of the Central Bank of Armenia (CBA) are provided daily with a one-day lag, while monthly data on the monetary survey are provided with three-week lag (and preliminary weekly data with a one-week lag). The balance sheets of the CBA and of the deposit money banks follow IAS methodology. Monthly interest rate data are provided with an one-week lag. 
9. Responding to a STA request, the CBA has compiled and submitted a complete set of monetary data beginning from December 2001 using Standardized Report Forms (SRF). STA validated the resulting monetary aggregates and the data have been published since the December 2006 issue of IFS Supplement and are used to update IFS. An Integrated Monetary Database (IMD) has also been established by STA to share the SRF data with MCD.

\section{External sector statistics}

10. The coverage of external sector data has improved in recent years. Trade statistics are provided on a timely basis, and trade data by origin, destination, and commodity are generally available within a month. Price data for exports and imports are less readily available. Quarterly balance of payments statistics are generally available with a three-month lag. However, on remittances, which account for a significant part of the inflows, there are considerable discrepancies among available source data. Survey data are considerably lower than data obtained through the money transfer system. The NSS and CBA are working on establishing a compilation program that would enable proper measurement of remittances. The absence of a comprehensive, continuously updated business register hampers the coverage of transactions and institutional units; in particular, the coverage of the financial account items for the private nonbank sector.

11. Quarterly data on international investment position are published by the NSS within one quarter after the reference period, and the annual data within two quarters; and are also provided for publication in IFS. 
Armenia: Table of Common Indicators Required for Surveillance

(As of June 2, 2009)

\begin{tabular}{|c|c|c|c|c|c|}
\hline & $\begin{array}{l}\text { Date of latest } \\
\text { observation }\end{array}$ & $\begin{array}{l}\text { Date } \\
\text { received }\end{array}$ & $\begin{array}{c}\text { Frequency } \\
\text { of } \\
\text { Data }^{7}\end{array}$ & $\begin{array}{l}\text { Frequency } \\
\quad \text { of } \\
\text { Reporting }^{7}\end{array}$ & $\begin{array}{c}\text { Frequency } \\
\text { of } \\
\text { publication }^{7}\end{array}$ \\
\hline Exchange Rates & June 2009 & $6 / 1 / 2009$ & $\mathrm{D}$ & $\mathrm{D}$ & $\mathrm{D}$ \\
\hline $\begin{array}{l}\text { International Reserve Assets and Reserve Liabilities of the Monetary } \\
\text { Authorities }^{1}\end{array}$ & May 2009 & $6 / 1 / 2009$ & $\mathrm{D}$ & $\mathrm{D}$ & M \\
\hline Broad Money & April 2009 & $5 / 25 / 2009$ & M & M & M \\
\hline Central Bank Balance Sheet & April 2009 & $5 / 25 / 2009$ & $\mathrm{D}$ & M & M \\
\hline Consolidated Balance Sheet of the Banking System & April 2009 & $5 / 25 / 2009$ & M & M & M \\
\hline Interest Rates ${ }^{2}$ & May 2009 & $5 / 29 / 2009$ & W & W & M \\
\hline $\begin{array}{l}\text { Revenue, Expenditure, Balance and Composition of Financing }{ }^{3}- \\
\text { Central Government }\end{array}$ & Apr. 2009 & $5 / 15 / 2009$ & M & M & Q \\
\hline $\begin{array}{l}\text { Stocks of Central Government and Central Government-Guaranteed } \\
\text { Debt }^{5}\end{array}$ & Q4 2008 & $1 / 30 / 2009$ & Q & Q & Q \\
\hline External Current Account Balance & Q4 08 & $3 / 30 / 2008$ & Q & Q & Q \\
\hline Exports and Imports of Goods and Services & Q4 08 & $3 / 30 / 2008$ & Q & Q & Q \\
\hline GDP/GNP & Q4 08 & $4 / 30 / 2009$ & Q & Q & Q \\
\hline
\end{tabular}

${ }^{1}$ Includes reserve assets pledged or otherwise encumbered as well as net derivative positions.

${ }^{2}$ Both market-based and officially determined, including discount rates, money market rates, rates on treasury bills, notes and bonds.

${ }^{3}$ Foreign, domestic bank, and domestic nonbank financing.

${ }^{4}$ The general government consists of the central government (budgetary funds, extra budgetary funds, and social security funds) and state and local governments.

${ }^{5}$ Including currency and maturity composition.

${ }^{6}$ Includes external gross financial asset and liability positions vis-à-vis nonresidents.

${ }^{7}$ Daily (D), Weekly (W), Monthly (M), Quarterly (Q), Annually (A); Irregular (I); and Not Available (NA). 


\section{INTERNATIONAL MONETARY FUND}

\section{Republic of Armenia-Update of Assessment of the Risks to the Fund and the Fund's Liquidity Position}

Prepared by the Finance and Strategy, Policy, and Review Departments

In consultation with other Departments

Approved by Andrew Tweedie and Ranil Salgado

June 17, 2009

1. This paper updates the assessment of the risks to the Fund and the effect on the Fund's liquidity position arising from the Stand-By Arrangement (SBA) for Armenia in view of its proposed augmentation. ${ }^{1}$ The authorities are requesting an augmentation of access equivalent to SDR 165.6 million (180 percent of quota) under the current 28-month SBA approved on March 6, 2009. Total augmented access under the arrangement would rise to about SDR 534 million (580 percent of quota). The augmentation would increase access available in 2009 and 2010, thereby making the arrangement more front-loaded (Table 1).

Table 1. Armenia: Proposed Augmentation under the SBA-Access and Phasing

\begin{tabular}{|c|c|c|c|c|c|c|c|}
\hline \multirow[b]{3}{*}{ Availability } & \multirow[b]{3}{*}{ Date } & & & \multicolumn{4}{|c|}{ Percent of quota } \\
\hline & & \multicolumn{2}{|c|}{ SDR $\mathrm{mn}$} & \multicolumn{2}{|c|}{ Purchase } & \multicolumn{2}{|c|}{ Cumulative } \\
\hline & & Original & Proposed & Original & Proposed & Original & Proposed \\
\hline \multirow[t]{5}{*}{2009} & March & 161.552 & 161.552 & 175.6 & 175.6 & 175.6 & 175.6 \\
\hline & May $1 /$ & 36.800 & & 40.0 & & 215.6 & \\
\hline & June (approval) & & 102.668 & & 111.6 & & 287.2 \\
\hline & August & 18.860 & 18.860 & 20.5 & 20.5 & 236.1 & 307.7 \\
\hline & November & 18.860 & 18.860 & 20.5 & 20.5 & 256.6 & 328.2 \\
\hline \multirow[t]{4}{*}{2010} & February & 23.552 & 48.485 & 25.6 & 52.7 & 282.2 & 380.9 \\
\hline & May & 23.552 & 48.485 & 25.6 & 52.7 & 307.8 & 433.6 \\
\hline & August & 23.552 & 48.485 & 25.6 & 52.7 & 333.4 & 486.3 \\
\hline & November & 23.552 & 48.485 & 25.6 & 52.7 & 359.0 & 539.0 \\
\hline \multirow[t]{3}{*}{2011} & February & 18.860 & 18.860 & 20.5 & 20.5 & 379.5 & 559.5 \\
\hline & May & 18.860 & 18.860 & 20.5 & 20.5 & 400.0 & 580.0 \\
\hline & Total & 368.000 & 533.600 & 400.0 & 580.0 & 400.0 & 580.0 \\
\hline
\end{tabular}

Source: Finance Department.

1/ May purchase was not made, and will therefore be available upon approval of the augmentation and completion of the first review.

\footnotetext{
${ }^{1}$ See Republic of Armenia-Assessment of the Risks to the Fund and the Fund's Liquidity Position (Country Report No. 09/140, 3/4/2009). Access under the existing arrangement exceeded both the annual and cumulative limits at the time. The Board subsequently doubled the normal GRA access limits to 200 percent of quota annually and 600 percent of quota cumulatively on March 24 . Under the proposed augmentation access would exceed the new annual access limit, indicating that an updated assessment is appropriate.
} 


\section{The Augmented ACCess Under the StAnd-By ArRAngement-Risks And IMPACT ON FUND'S FINANCES}

2. The proposed augmentation would result in access under the arrangement surpassing the annual access limit and would make the Fund Armenia's predominant source of external financing. If all purchases are made as scheduled:

- Armenia's outstanding use of GRA resources would exceed 200 percent of quota by end-June 2009, and would peak at 580 percent of quota in May 2011. In terms of quota, this projected peak exposure would be above the median for recent exceptional access cases (Figure 1). Including outstanding PRGF loans, the peak exposure would be 637 percent of quota (SDR 586 million) in May 2011.

\section{Figure 1. Fund Credit Outstanding in the GRA around Peak Borrowing 1/}

\section{(In percent of quota)}

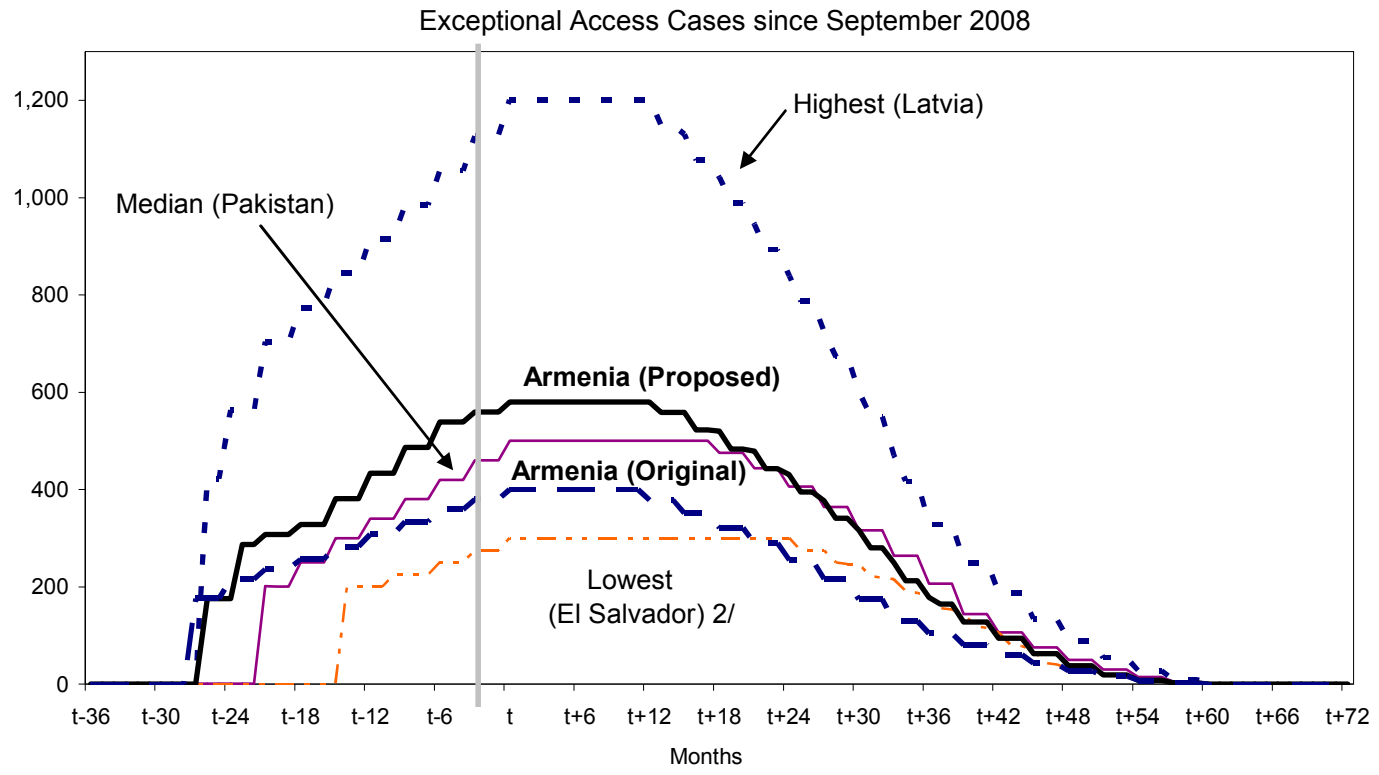

Source: IFS, Finance Department, and IMF staff estimates.

1/ Peak borrowing ' $t$ ' is defined as the highest level of credit outstanding for a member. Repurchases are assumed to be on an obligations basis.

2/ The authorities have expressed their intention to treat the arrangement as precautionary, as balance of payments pressures have not materialized.

- $\quad$ GRA credit outstanding to Armenia would reach 8.5 percent of GDP and about 55 percent of reserves by end-2011 (Table 2). This peak relative to GDP would exceed the median of the recent exceptional access cases, while the peak relative to reserves would be the second highest (Figure 2). 
Table 2. Armenia-Capacity to Repay Indicators 1/

\begin{tabular}{|c|c|c|c|c|c|c|c|c|}
\hline & June-09 & 2009 & 2010 & 2011 & 2012 & 2013 & 2014 & 2015 \\
\hline \multicolumn{9}{|l|}{ Exposure and Repayments (In SDR millions) } \\
\hline $\begin{array}{l}\text { GRA credit to Armenia } \\
\text { (In percent of quota) } \\
\text { Charges due on GRA credit 2/ } \\
\text { Debt service due on GRA credit 2/ }\end{array}$ & $\begin{array}{r}264.2 \\
(287.2)\end{array}$ & $\begin{array}{r}301.9 \\
(328.2) \\
3.1 \\
3.1\end{array}$ & $\begin{array}{r}495.9 \\
(539.0) \\
9.1 \\
9.1\end{array}$ & $\begin{array}{r}533.6 \\
(580.0) \\
13.3 \\
13.3\end{array}$ & $\begin{array}{r}445.0 \\
(483.7) \\
13.3 \\
102.0\end{array}$ & $\begin{array}{r}257.7 \\
(280.1) \\
9.2 \\
196.5\end{array}$ & $\begin{array}{r}86.5 \\
(94.1) \\
3.2 \\
174.3\end{array}$ & $\begin{array}{r}7.1 \\
(7.7) \\
0.8 \\
80.2\end{array}$ \\
\hline \multicolumn{9}{|l|}{ Debt and Debt Service Ratios 3/ } \\
\hline $\begin{array}{l}\text { In percent of GDP } \\
\text { External debt, public } \\
\text { GRA credit to Armenia } \\
\text { Public external debt service } \\
\text { Debt service due on GRA credit }\end{array}$ & $\begin{array}{r}15.3 \\
3.3 \\
0.5\end{array}$ & $\begin{array}{r}31.7 \\
5.0 \\
0.8 \\
0.1\end{array}$ & $\begin{array}{r}38.9 \\
8.3 \\
1.1 \\
0.2\end{array}$ & $\begin{array}{r}39.5 \\
8.5 \\
1.1 \\
0.2\end{array}$ & $\begin{array}{r}36.1 \\
6.6 \\
2.4 \\
1.5\end{array}$ & $\begin{array}{r}32.1 \\
3.5 \\
3.5 \\
2.7\end{array}$ & $\begin{array}{r}28.2 \\
1.1 \\
2.9 \\
2.2\end{array}$ & $\begin{array}{r}25.7 \\
0.1 \\
2.1 \\
0.9\end{array}$ \\
\hline $\begin{array}{l}\text { In percent of Gross International Reserves } \\
\text { External debt, public } \\
\text { GRA credit to Armenia } \\
\text { Public external debt service } \\
\text { Debt service due on GRA credit }\end{array}$ & $\begin{array}{r}129.4 \\
28.3 \\
3.9\end{array}$ & $\begin{array}{r}184.6 \\
29.2 \\
4.6 \\
0.3\end{array}$ & $\begin{array}{r}225.9 \\
48.1 \\
6.1 \\
0.9\end{array}$ & $\begin{array}{r}255.7 \\
55.3 \\
7.3 \\
1.4\end{array}$ & $\begin{array}{r}253.0 \\
46.6 \\
17.0 \\
10.7\end{array}$ & $\begin{array}{r}210.7 \\
23.3 \\
23.2 \\
17.7\end{array}$ & $\begin{array}{r}178.1 \\
6.8 \\
18.5 \\
13.8\end{array}$ & $\begin{array}{r}162.7 \\
0.5 \\
13.2 \\
5.8\end{array}$ \\
\hline $\begin{array}{l}\text { In percent of Exports of Goods and Services } \\
\text { External debt, public } \\
\text { GRA credit to Armenia } \\
\text { Public external debt service } \\
\text { Debt service due on GRA credit }\end{array}$ & $\begin{array}{r}105.5 \\
23.1 \\
3.2\end{array}$ & $\begin{array}{r}232.7 \\
36.8 \\
5.8 \\
0.4\end{array}$ & $\begin{array}{r}235.2 \\
50.1 \\
6.4 \\
0.9\end{array}$ & $\begin{array}{r}215.7 \\
46.7 \\
6.2 \\
1.2\end{array}$ & $\begin{array}{r}184.1 \\
33.9 \\
12.4 \\
7.8\end{array}$ & $\begin{array}{r}157.2 \\
17.4 \\
17.3 \\
13.2\end{array}$ & $\begin{array}{r}134.3 \\
5.2 \\
14.0 \\
10.4\end{array}$ & $\begin{array}{r}120.4 \\
0.4 \\
9.8 \\
4.3\end{array}$ \\
\hline $\begin{array}{l}\text { In percent of Central Government Revenue (ex } \\
\text { Public external debt service } \\
\text { Debt service due on GRA credit }\end{array}$ & ts) & $\begin{array}{l}4.2 \\
0.3\end{array}$ & $\begin{array}{l}5.4 \\
0.8\end{array}$ & $\begin{array}{l}5.7 \\
1.1\end{array}$ & $\begin{array}{r}12.0 \\
7.5\end{array}$ & $\begin{array}{l}22.4 \\
17.1\end{array}$ & $\begin{array}{l}18.1 \\
13.5\end{array}$ & $\begin{array}{r}12.5 \\
5.6\end{array}$ \\
\hline $\begin{array}{l}\text { In percent of Total Public External Debt } \\
\text { GRA credit to Armenia } \\
\text { Armenia's total debt to the Fund 4/ }\end{array}$ & & $\begin{array}{l}15.8 \\
19.6\end{array}$ & $\begin{array}{l}21.3 \\
23.8\end{array}$ & $\begin{array}{l}21.6 \\
23.4\end{array}$ & $\begin{array}{l}18.4 \\
19.6\end{array}$ & $\begin{array}{l}11.0 \\
11.8\end{array}$ & $\begin{array}{l}3.8 \\
4.3\end{array}$ & $\begin{array}{l}0.3 \\
0.6\end{array}$ \\
\hline $\begin{array}{l}\text { In percent of Total Public External Debt Service } \\
\text { Debt service due on GRA credit } \\
\text { Armenia's total debt service to the Fund 4/ }\end{array}$ & & $\begin{array}{r}6.5 \\
39.2\end{array}$ & $\begin{array}{l}14.4 \\
37.3\end{array}$ & $\begin{array}{l}18.8 \\
39.6\end{array}$ & $\begin{array}{l}62.6 \\
71.8\end{array}$ & $\begin{array}{l}76.4 \\
80.8\end{array}$ & $\begin{array}{l}74.4 \\
77.7\end{array}$ & $\begin{array}{l}44.3 \\
46.8\end{array}$ \\
\hline
\end{tabular}

Sources: Armenian authorities, Finance Department, World Economic Outlook, and IMF staff estimates.

$1 /$ Given the focus of the risk assessment, and for the sake of comparability with other exceptional access cases, the capacity to repay indicators presented in this table include only the GRA-related items, unless otherwise indicated. Assumes full drawings.

2/ Includes surcharges and service fees.

3/ Staff projections for external debt, GDP, gross international reserves, and exports of goods and services, as used in the staff report that requests the proposed

SBA. For June 2009, projections for external debt, GDP, gross international reserves, and exports of goods and services are for 2008.

4/ Total debt to the Fund comprises balances outstanding on GRA credit and PRGF loans. 
Figure 2. Peak Fund Exposure and Debt Service Ratios for Recent Exceptional Access Cases

Peak GRA Exposure Ratios

A. In Percent of GDP

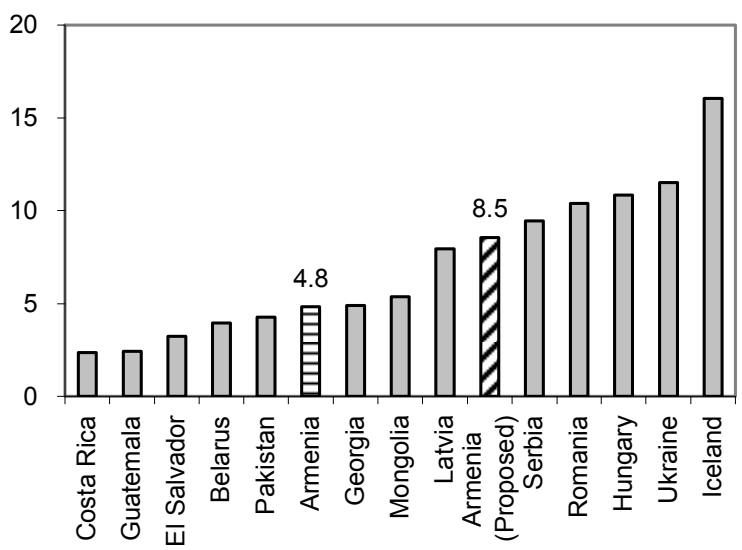

B. In Percent of Gross International Reserves

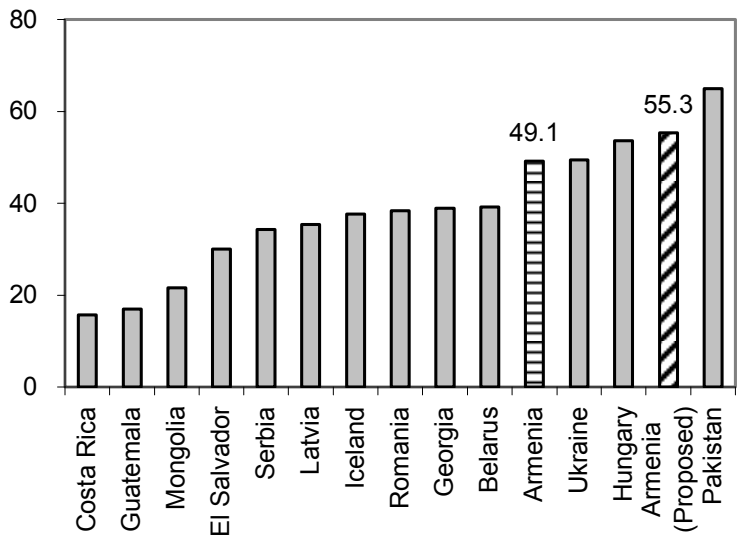

C. In Percent of Total External Debt

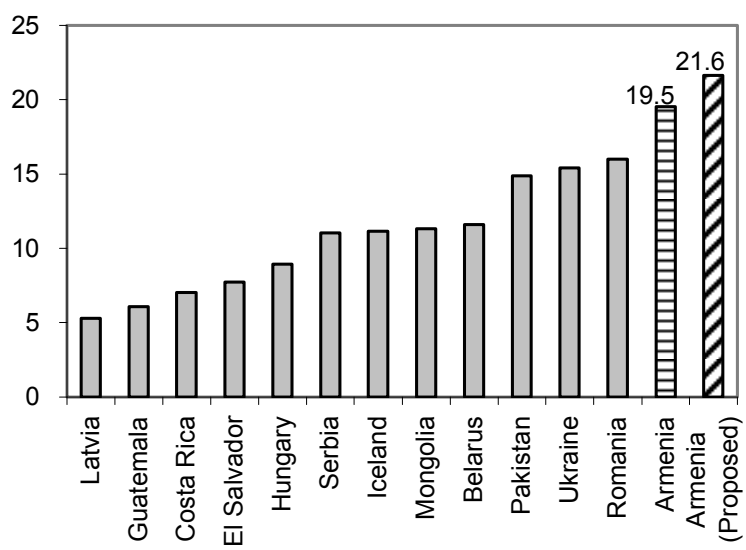

Peak Debt Service Ratios

A. Total External Debt Service in Percent of Exports of Goods and Services

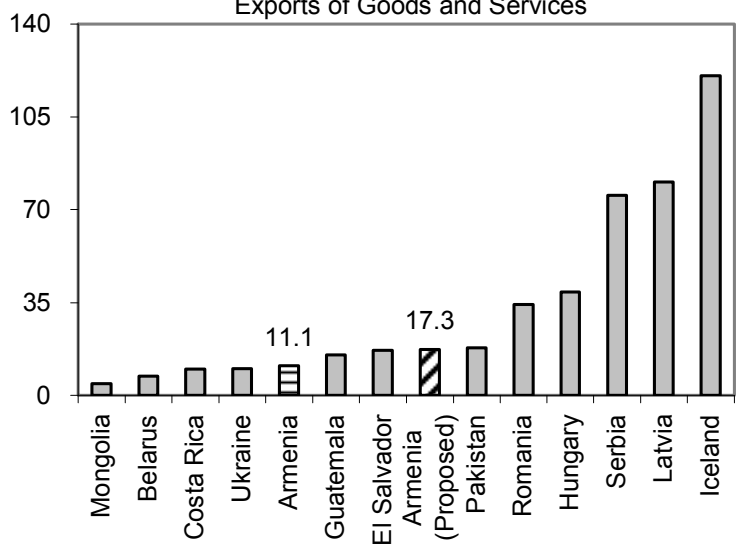

B. Debt Service to the GRA in Percent of Exports of Goods and Services

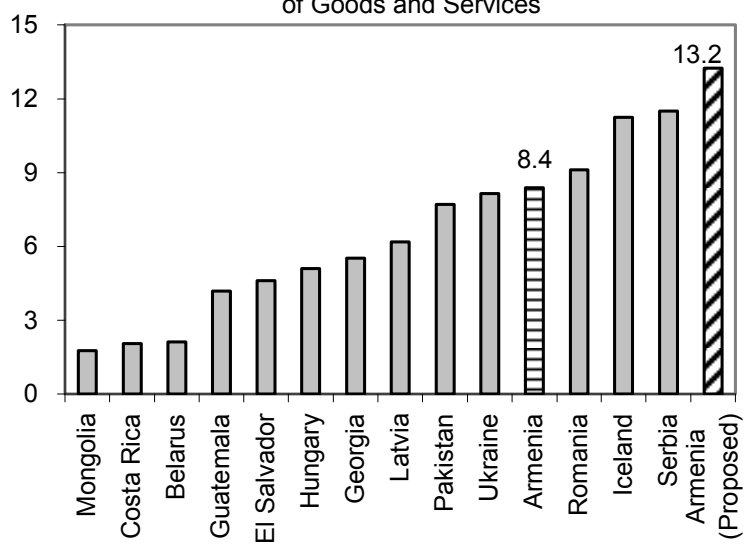

C. Debt Service to the GRA in Percent of Total External Debt Service

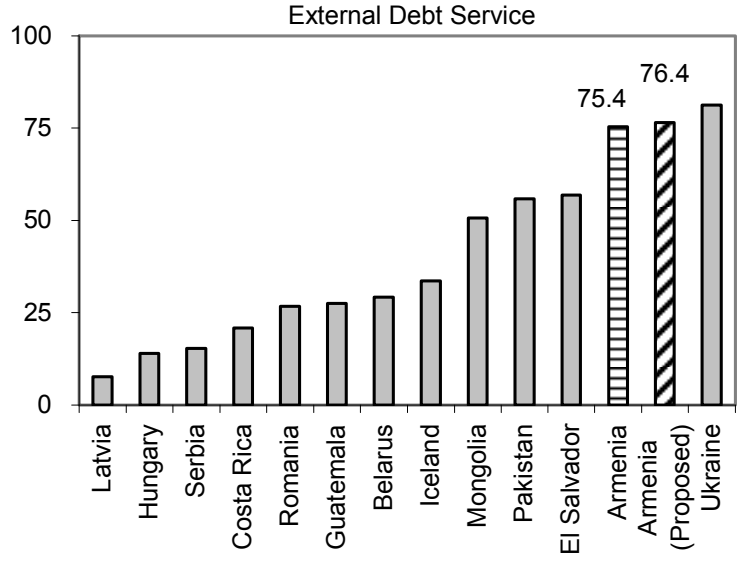

Source: Armenian authorities and IMF staff estimates, and World Economic Outlook. 
3. The proposed augmentation would raise Armenia's debt service ratios to the Fund to high levels, including relative to government revenues, amid a sharp increase in Armenia's public indebtedness (Table 2 and Figure 2): ${ }^{2}$

- $\quad$ External public debt would jump to almost 40 percent of GDP in 2010-11, from only 15 percent currently, lifting total public debt to almost 46 percent of GDP.

- The peak in GRA credit as a share of external debt would remain the highest among recent exceptional access cases at close to 22 percent. (The modest increase in this ratio compared with the original program reflects the sizeable projected increase in other external debt).

- $\quad$ At its 2013 peak, total public external debt service would reach 23 percent of reserves, 17 percent of exports of goods and services, and 22 percent of central government revenue (excluding grants).

- $\quad$ Debt service to the GRA would peak in 2013 at about three-quarters of total external debt service, the second highest among the recent exceptional access cases. In terms of exports of goods and services, debt service to the GRA would peak at 13 percent in 2013, the highest among the recent exceptional access cases. Even with remittances added to goods and services exports, the ratio would still exceed 8 percent.

4. The impact on the Fund's finances would remain contained by the comparatively small scale of access in SDR terms. The augmentation would reduce the one-year forward commitment capacity (FCC) by $1 / 2$ percent, and would represent a modest increase in GRA exposure in relation to the Fund's current level of precautionary balances.

\section{ASSESSMent}

5. Augmentation of access under the SBA aims to avert severe adjustment under the worsened economic outlook for 2009-10, but there are significant risks to the program in the near term. Many of the downside risks identified at the time of the SBA request, particularly a much deeper downturn in Russia, have materialized. The resulting collapse in revenues has led to additional financing needs to prevent excessive adjustment. Armenia remains very vulnerable to a further deterioration of global conditions, particularly in Russia. Balance of payments inflows, including remittances, could turn out to be lower than projected, implying risks to the international reserves target and forcing further adjustment in the exchange rate, which would further increase debt and debt service ratios.

6. Repayment risks have increased relative to the outset of the program, though the still comparatively small scale of access in absolute terms would contain the potential adverse impact on the Fund's finances. Debt service to the Fund is expected to become

\footnotetext{
${ }^{2}$ Debt service to the Fund is calculated assuming that all purchases and repurchases are made as scheduled and surcharges are calculated according to the current schedule. The new system of surcharges will go into effect on August 1, 2009, subject to grandfathering at the member's request.
} 
large relative to current receipts, international reserves and central government revenues, and to account for three-quarters of total external debt service. Near-term risks could increase these ratios. In these conditions, the authorities' commitment to firm implementation of the program to underpin an improved macroeconomic situation in the medium term is essential to safeguarding Fund resources. 
Press Release No. 09/228

International Monetary Fund

FOR IMMEDIATE RELEASE

Washington, D.C. 20431 USA

June 22, 2009

\section{IMF Completes First Review Under Stand-By Arrangement with Armenia and Approves Increase in Financial Support to US\$822.7 Million}

The Executive Board of the International Monetary Fund (IMF) today completed the first review of Armenia's economic performance under a Stand-By Arrangement (SBA) and approved an increase in the IMF's financial support to an amount equivalent to SDR 533.6 million (about US\$822.7 million; or 580 percent of Armenia’s quota). These decisions enable the immediate release of SDR 102.7 million (about US $\$ 158.3$ million), bringing the total disbursed to SDR 264.2 million (about US\$400 million). The Board also granted a waiver of performance criteria on net banking system credit to the government and the program's fiscal balance.

The revised arrangement will support the government's economic program amid a sharperthan-expected impact from the global financial crisis. The 28-month SBA was approved on March 6 (see Press Release 09/68).

The key objectives of the program are to help Armenia adjust to the external shock, maintain confidence in the currency and the financial system, and protect the poor. The sharp contraction in economic activity, the fall in remittances, an increase in unemployment, and difficult conditions in credit markets require an easing of macroeconomic policies and the implementation of several measures to stimulate domestic demand and create new jobs.

The main policies under the revised program are:

- Monetary conditions will be eased, including by widening the range of central bank instruments to provide longer-term dram liquidity to banks, and by increasing targeted onlending to small and medium enterprises.

- $\quad$ Fiscal policy will also be eased. Despite the fall in fiscal revenue, the government will aim to maintain its overall expenditure at a level close to the original 2009 budget, protecting 
social spending while increasing expenditure on high-priority infrastructure projects, financed in large part by bilateral donors.

- $\quad$ Reforms will focus in particular on the continued strengthening of financial sector supervision and improving tax administration.

- Social safety nets will be enhanced by targeting social services to the poor.

Following the Executive Board's discussion on Armenia, Mr. Murilo Portugal, Deputy Managing Director and Acting Chair, stated: "Since the approval of the stand-by arrangement in March 2009, the external economic outlook has deteriorated significantly for Armenia. Falling private transfers and capital inflows have aggravated external imbalances and affected household incomes and investor confidence. Construction activity, the main driver of growth in previous years, has collapsed, and the economy is experiencing a deep contraction.

"The additional financial assistance from the Fund will help cover Armenia's growing financing needs, while the recalibration of the authorities' economic program will help them better respond to the deepening downturn. The program envisages an easing of monetary and fiscal policy to mitigate the severity of the crisis, while laying the ground for future fiscal consolidation primarily through one-off investment expenditures and measures to strengthen tax policy and administration. The authorities remain firmly committed to achieving the program's objectives of adjusting to the changed external environment, supporting confidence in the currency and the banking system, and protecting the poor.

"Following the successful return to a flexible exchange rate, monetary policy will focus on maintaining low inflation. With the fall in inflation rates, the recent reductions of policy interest rates are appropriate. In addition, the authorities are taking active measures to provide liquidity to the banking system and help resume lending. Fiscal policy will provide crucial support by accelerating growth-enhancing investment in infrastructure and strengthening social safety nets.

"As external conditions improve in 2010, growth is expected to resume gradually. The shortterm outlook remains, however, very challenging. Continued reforms will be necessary to boost the medium-term growth potential of the economy, including efforts to improve the business climate, completion of the unfinished tax policy and tax administration reform agenda, and progress on financial sector reforms." 


\section{Statement by Age Bakker, Executive Director for the Republic of Armenia June 22, 2009}

I would like to thank staff and management for their prompt reaction to the downside risks materializing. The risks, identified at the time of the SBA request, stem from the impact of the global and regional economic slowdown on Armenia and call for a recalibration of the program. The steep contraction of exports and remittances, as shown in the staff report, clearly points to growing problems for Armenia. Together with a considerably worsened growth outlook, a larger than originally foreseen external financing gap has emerged.

The Fund is expected to cover part of this additional gap, with bilateral and multilateral donors and creditors also stepping in. Additional financing needs are particularly high for 2009 and 2010. The objectives of the program remain intact, and the government will take additional anti-crisis measures, including implementing fiscal and structural reforms that would ensure debt sustainability going forward. Absent additional Fund financing, Armenia would have limited options to finance the remaining gap and experience protracted BoP problems with a sharp fall in domestic demand, leading to an even deeper economic contraction.

I would like to emphasize the good track record of the Armenian authorities - who have generally held the program on track - and the external nature of the economic shock they face. It may be useful to note that Armenia was considered a heavily indebted nation only 10 years ago, but managed to get much of its debt reduced and under control without any debt forgiveness, just by implementing sound policies. Before the crisis, the debt service ratio was brought to under 3 percent of exports, so the starting position for Armenia to take the official debt on board is actually quite good. Private sector external liabilities are unusually small, at 5 percent of GDP. Under the program, the debt may increase to slightly above 40 percent of GDP if full disbursements of external funds are made, which is smaller than in most other program cases. Staff has calculated that the debt position would remain sustainable under various shock scenarios.

I also believe that the temporary nature of the Fund's financing is well preserved. In this respect, it certainly helps that the major structural commitments under the program have been strengthened, with deepened reforms in the areas of tax administration and tax policy.

The rather small size of the financial sector suggests that there is a very small risk from any contingent recapitalization efforts. Armenian banks are extremely well capitalized - to an extent of more than double the regulatory requirement of 12 percent, which serves as a useful buffer with non-performing loans rising in line with the slowdown in economic activity. Contrary to the expectations of many observers, the substantial depreciation of the national currency, the dram, has not undermined confidence in banks and has not created liquidity 
pressures, though increased dollarization has contributed to a rapid slowdown in credit activity. Dollarization also weakened the monetary transmission mechanism somewhat. Nevertheless, the authorities intend to resume their inflation targeting policies by, inter alia, gradually adjusting the policy rate, and, so far, they seem to be on track to meeting their objective. Higher reserves will undoubtedly contribute to greater stability going forward. The authorities would also continue to guard financial sector stability by strengthening the deposit guarantee fund.

On the fiscal side, tax revenues have fallen significantly, leading to delays in budgeted government spending that compound the drop in private sector demand. The authorities are committed to preserving essential social spending and protecting the poor, as envisaged under the original program.

Bilateral support would allow for the external financing of some of the job-creating capital expenditures, such as road rehabilitation, and critical housing construction in the earthquake affected areas, while safeguarding the budgeted social spending would imply an increase in deficit. Keeping spending in nominal terms, as planned under the original program with a substantial fall in nominal GDP, would result in a deficit of a little over 6 percent of GDP and just under 6 percent in 2010 .

The authorities understand the need to maintain a sustainable fiscal position in the medium term. Their tax reform would result in increased revenues. The World Bank is helping to further strengthen the targeting of the social safety nets, but generating additional sustainable revenues and savings will require some time. The authorities' tax administration reform continues to be the crucial element of the program. Already in the first quarter of 2009, the stock of the tax credits was reduced and better VAT practices are being implemented. The tax administration reforms were also launched in additional areas - tax filing, restructuring of the regional tax offices - and electronic filing will be introduced shortly. Some revenueenhancing tax policy measures on the tobacco-presumptive taxes were also introduced. Analytical work on the medium-term fiscal risks has been upgraded.

The government is also taking measures to improve the business climate and enhance competitiveness. There seem to be some additional privatization-related FDI possibilities for some of the industries in Armenia that have not yet been privatized, especially if the business climate and the tax/customs regime improvements are made. The FDI prospects would also improve with greater regional stabilization and cooperation.

There exist further possibilities of downside risks to the program in case of an even weaker growth outcome for this year or greater deterioration in terms of trade. The authorities were discussing with staff the scenarios of what needs to be done in case the already bad outlook deteriorates further. The institutional and political capacity to deliver stronger adjustment efforts is definitely present in Armenia, but the Fund's attitude has rightly been focused on 
the appropriate combination of adjustment and financing, reflecting the financing needs as well as a burden sharing. In this respect, I believe the staff's proposals strike the right balance at the moment, and I see the core mission of this SBA (as a successor to the PRGF) as avoiding a reversal of Armenia's success in poverty alleviation. Thus, in order to preserve the good reputation of the IMF in Armenia and in the region in general, there is a need for some degree of flexibility from the Fund in case any of the risks (be it upside or downside) materialize. Armenia, like only a few other countries, gets substantial and steady support from the large Diaspora abroad. The possible reopening of the border with Turkey is another sizeable upside risk to the outlook. 